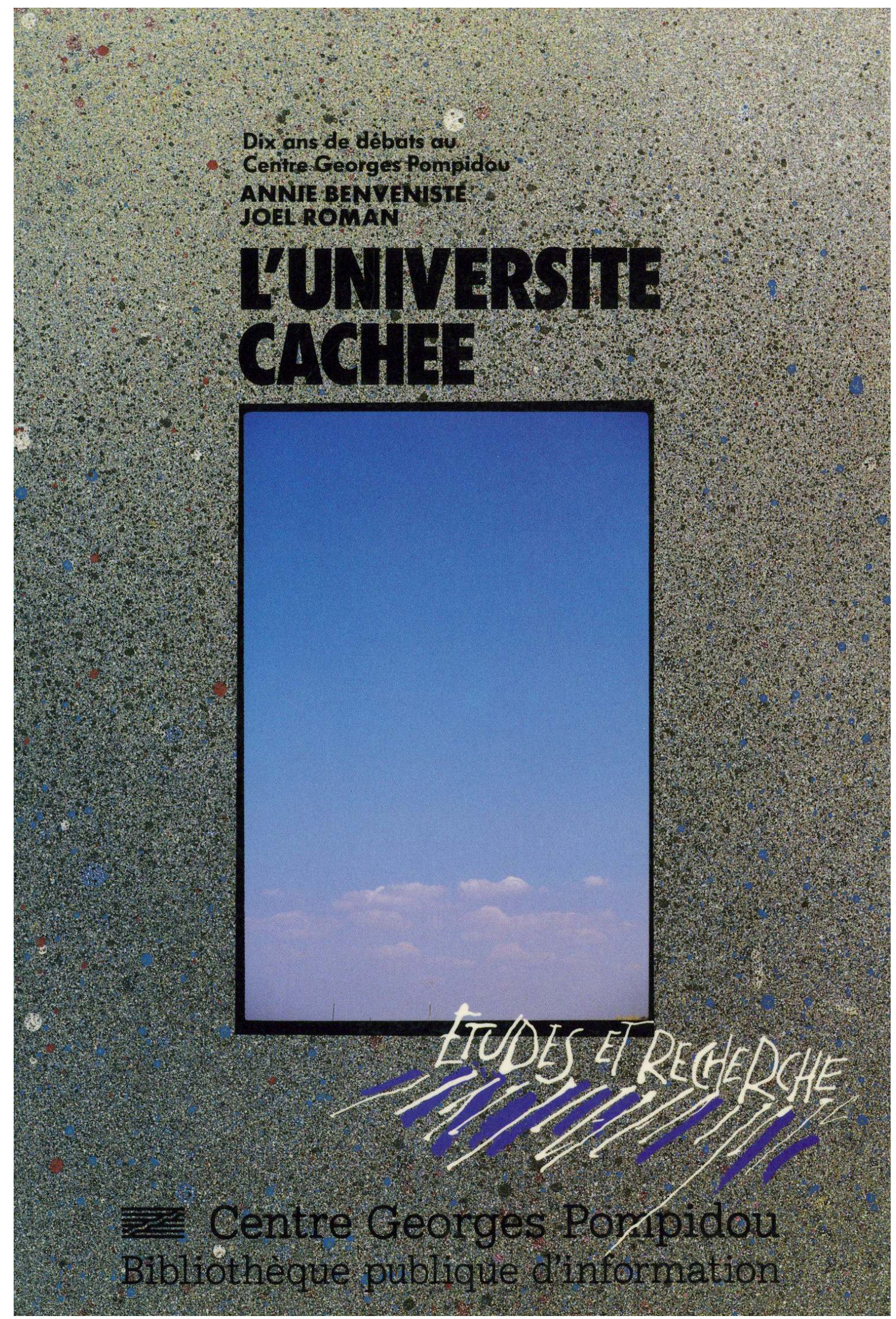




\section{L'université cachée}

Dix ans de débats au Centre Georges Pompidou

\section{Annie Benveniste et Joel Roman}

DOI : 10.4000/books.bibpompidou.2286

Éditeur : Éditions de la Bibliothèque publique d'information Année d'édition : 1990

Date de mise en ligne : 15 janvier 2019

Collection : Études et recherche

ISBN électronique : 9782842462291

\section{Donerstition ebooks}

http://books.openedition.org

\section{Édition imprimée}

ISBN : 9782902706310

Nombre de pages : 115

\section{Référence électronique}

BENVENISTE, Annie ; ROMAN, Joel. L'université cachée : Dix ans de débats au Centre Georges Pompidou. Nouvelle édition [en ligne]. Paris : Éditions de la Bibliothèque publique d'information, 1990 (généré le 02 février 2021). Disponible sur Internet : <http://books.openedition.org/bibpompidou/2286>. ISBN : 9782842462291. DOI : https://doi.org/10.4000/books.bibpompidou.2286.

Ce document a été généré automatiquement le 2 février 2021. Il est issu d'une numérisation par reconnaissance optique de caractères.

(c) Éditions de la Bibliothèque publique d'information, 1990

Conditions d'utilisation:

http://www.openedition.org/6540 
Dix ans de débats au Centre Georges Pompidou : parole circulante dans l'espace public, ou parole, autorisée- dans des lieux circonscrits?

Une histoire avec ses excès et ses remises en ordre, et des enjeux aussi. Qu'il s'agisse de philosophie, de sciences sociales, de littérature ou d'architecture, la polyphonie des débats traduit au-delà d'une volonté de diffusion propre à chaque institution culturelle, la place singulière occupée par le Centre Georges Pompidou dans le champs culturel. À l'intersection du " grand public » et des « créateurs », du loisir et du savoir, les débats ont progressivement renoncé pour dessiner un espace de « légitimité » spécifique.

Sous le « déluge des paroles » ni totalement médiatique ni classiquement universitaire, les débats du Centre organisent à leur manière des cycles de "formation" qui sans souci de diplômes, esquissent des voies de transmission «sauvages». Université cachée à la mesure du retrait de l'université traditionnelle, Centre Georges Pompidou serait ainsi une institution mutante capable de répondre à de nouveaux besoins et, d'anticiper les reclassements disciplinaires à venir.

\section{ANNIE BENVENISTE}

Sociologique. Elle a récemment publié le Bosphore à la Roquette, L’Harmattan, 1990

\section{JOEL ROMAN}

Agrégé de philosophie, corédacteur en chef de la revue Esprit. Il a signé avec Laurence Cornu et Jean Claude Pompougnac Le Barbare et l'écolier, Calmann Lévy, 1990. 


\section{SOMMAIRE}

Introduction

Chapitre 1. Évolution historique : dix ans de débats à Beaubourg

La période des commencements (1978-1982)

Généralisation des débats (1982-1985)

Mise en ordre des débats (1985-1989)

Chapitre 2. Les thématiques et les styles de débats : du spectacle à la conférence magistrale Analyse des modèles : le débat-spectacle

Le débat-actualité

Le débat-colloque

Les conférences

\section{Chapitre 3. Les acteurs des débats}

Les organisateurs

Les animateurs

Les participants

Le public

Chapitre 4. Les débats du Centre dans le champ culturel

Les fonctions du Centre dans le champ culturel

La structure de l'espace public

Conclusion

Annexes

Remerciements 


\section{Introduction}

1 Dans le rapport qu'il avait consacré au Centre Georges Pompidou, Michel de Certeau parlait de l'« impérialisme du visuel » qui y aurait régné. Il écrivait notamment : « Le plateau de Beaubourg, c'est l'exorbitation du visible, le sabbat encyclopédique du voir. Entre producteurs et consommateurs, une même logique fonctionne: d'un côté, montrer; de l'autre, voir.

2 «De ce point de vue, ce qui manque, c'est du secret, c'est de l'ombre, de l'invisible, et donc aussi la séduction qu'instaure le caché. On sait également l'antinomie qui oppose l'auditif au visuel. L'audiovisuel fait coïncider des contraires, et de fait, sur le théâtre de Beaubourg comme en une guerre des dieux, l'image semble combattre le son: chaque sens a ses forteresses et ses clientèles. L'IRCAM mis à part ou la "Revue parlée", il manque à ces lieux une musique; à ces vues, des voix qui les muent, comme disait Marguerite Duras, en "films pour des voix" où entendre ce qu'on ne peut voir; à la lecture même des signes, la mise en jeu plus systématique des concerts ou des poétiques de l'écriture ${ }^{1}$.»

3 L'étude des débats est-elle à même d'infléchir ce constat ? A l'évidence, l'opposition du voir et de l'entendre les néglige : que de paroles n'y a-t-on pas entendues pendant plus de dix ans de débats! Et pourtant, les débats sont bien aussi dans l'espace du visible, configurent leur propre espace scénique. La parole n'y est pas le versant d'ombre du visible, comme semblait le souhaiter de Certeau, mais au contraire son double insistant : parole exposée, parole à voir, le débat participe d'un espace plus large, qu'il révèle et modifie parfois. C'est aux modalités de cette révélation et aux figures de ces modifications que s'est attachée cette étude. Nous espérons avoir un peu déporté le jugement de Michel de Certeau, avoir rendu justice à la séduction propre de cette publicité, et avoir donné à entendre sinon une poétique du débat, du moins ce que pourrait être sa rythmique.

4 L'étude demandée comportait deux volets : celui de la thématique et de la place des débats dans le champ culturel, celui de l'ethnographie et de la scénographie des débats. Très vite, il nous est apparu qu'il était nécessaire de croiser ces approches : les rituels de présentation de soi, par exemple, marquent la place de chacun dans le champ mieux que ne le ferait un curriculum vitae ; inversement, les thèmes de débats ne manquent pas d'imposer des codes spécifiques, et une typique. D'où le choix d'une scansion en quatre parties : une ouverture historique, qui s'efforce à une périodisation dans l'organisation 
des débats, laquelle oriente aussi l'étude des styles, dans la mesure où chaque grande période correspond à la prévalence d'un style; la deuxième partie s'imposait donc. Il était alors loisible de questionner successivement les acteurs des débats, leurs stratégies, leurs enjeux, avant de revenir dans une dernière partie à l'ensemble du champ, et interroger les effets des débats.

5 La méthode choisie a combiné l'analyse des archives, l'entretien approfondi avec les organisateurs, animateurs, intervenants de débats et l'observation ethnologique d'un échantillon de manifestations. Pour recueillir les premières données, nous nous sommes référés aux bulletins de programmation et aux programmes hebdomadaires qui nous permettaient d'étudier à la fois le nombre et la nature des débats en fonction des instances organisatrices et la place de ces débats dans l'ensemble des manifestations du Centre. Cette photographie a permis un premier repérage qu'il a fallu compléter, pour mieux connaître le type des intervenants et des thématiques, par l'étude de la documentation archivée par chaque instance. Un premier biais a pu apparaître, à ce niveau-là, étant donné l'inégale importance accordée aux archives par chaque service d'une part, en fonction du type de manifestations recensées d'autre part. La Bpi, de par la formation de son personnel, est ainsi plus "naturellement» tournée vers l'archivage que le CCI. Quant au musée, il a engagé à partir de 1981 une personne qui eut pour tâche spécifique d'organiser des débats. Mais ce qui est du domaine de l'actualité et de l'éphémère, comme les débats, entraîne moins souvent des pratiques de conservation que les expositions, plus prestigieuses. Les manifestations importantes font cependant l'objet d'articles de presse.

6 Nous avons ainsi mis au jour onze ans de débats, de 1978 à 1989. Les entretiens ont concerné également l'ensemble de la période. Ils sont donc à analyser comme des représentations qui ont parfois subi le travail de la mémoire: occultation, réinterprétation. Toutefois, nous n'avons pas suspecté les représentations des uns et des autres par principe: elles font partie intégrante des dynamiques concernées, entraînant des stratégies, des inflexions, des choix. Mieux, nous avons délibérément rejeté l'hypothèse fallacieuse d'une saisie «objective» du débat: notre propre inscription dans le même champ que celui des acteurs des débats (il nous est même arrivé d'en être) l'aurait interdit, mais aussi l'impossibilité de déterminer à coup sûr le point de vue sur le débat qui serait le "vrai " point de vue. Comme toute pratique sociale, le débat se diffracte dans autant de regards que de postures pratiques, dont nul n'a de privilège a priori. En revanche, certains d'entre eux sont davantage suivis d'effets que d'autres : c'est le cas des organisateurs et des participants. Nous rencontrons ici la question du public, postulé par chacun des acteurs comme l'horizon de son intervention. Sauf quand il intervient, pour aussitôt se dissocier et prendre alors des visages déterminés ( $c f$. chapitre 4 ), le public est moins un acteur qu'une postulation commune aux acteurs. Une autre étude serait nécessaire pour cerner ses attentes, ses réactions, ses postures propres.

7 L'observation ethnologique a porté sur des manifestations récentes qui ont eu lieu au moment de l'étude. L'échantillon a été construit en fonction de la situation d'enquête. On a essayé de suivre aussi bien des manifestations brèves que des journées, des premières que des rencontres plus ancrées dans la tradition du Centre. Ce troisième type de données nécessite une explicitation. A travers ce qu'on a appelé une " ethnographie des débats », il s'agit de comprendre leur mode de structuration. Toute rencontre obéit à des règles, des conventions qui servent à " orienter et organiser » la 
communication ${ }^{2}$ (Les pratiques de l'échange verbal constituent un véritable «ordre rituel ». On peut procéder à un découpage des rencontres en unités structurelles et situer les différents types de débats à l'intérieur d'une analyse des rituels d'interaction. La mise en scène du débat est fonction des lieux. Elle est aussi fonction des médiations qui introduisent les acteurs de la rencontre. L'animateur propose les règles de la prise de parole. C'est dans la façon dont elles sont respectées, déjouées ou transgressées que se lisent les différents types de débats. Une étude des interactions verbales permet d'analyser le système des pratiques qui organisent la communication.

Enfin, l'étude invitait à construire des points de comparaison : certains s'imposaient, qui se proposaient des tâches analogues de médiation culturelle, faisant souvent intervenir les mêmes participants. Les débats de France-Culture et de la Fnac nous ont ainsi servi de point d'appui. Quant à rapporter les débats au champ culturel dans son ensemble, nul autre guide n'était plus indiqué que sa fréquentation assidue.

On le voit, la méthode suivie est composite : le double aspect de la recherche, joint à la dimension protéiforme de l'objet, l'exigeait. Nous ne croyons pas avoir concédé à la diversité des approches la rigueur des descriptions et des argumentations. Au contraire, celle-ci a permis plus d'une fois de vérifier le bien-fondé d'une hypothèse, de tels recoupements garantissant contre les effets trop prégnants d'une méthodologie unique.

Nous n'avons pour autant pas épuisé le sujet, loin de là. On a déjà signalé la part d'ombre de l'étude des publics. Mais même au sein de l'épure retenue, des questions restent ouvertes. Curieusement, la fonction des débats n'a pas pu être dégagée de manière univoque: c'est qu'ils en cumulent plusieurs, et que d'un débat à l'autre, l'accent peut changer. C'est aussi que les effets excèdent toujours les intentions. Il nous semble néanmoins que les conclusions auxquelles nous sommes parvenus, pour provisoires qu'elles doivent être tenues, contribuent à jeter quelque éclairage sur la manière dont opère le Centre dans le champ culturel, voire sur la structure de celui-ci. Si elles renvoient aux acteurs, et d'abord aux organisateurs, une image un peu plus précise des possibles qui s'offrent à eux, nous estimerons avoir rempli notre rôle. Le reste, qui est peut-être le plus important, n'est que l'écho d'un bruissement de paroles. Nous avons réchauffé quelques paroles gelées : avant que l'inertie du froid ne vienne les ressaisir, prêtons encore un peu l'oreille à cette polyphonie.

\section{NOTES}

1. Michel de Certeau, «Le sabbat encyclopédique du voir », Esprit, février 1987, p. 70-71.

2. Erving Goffman, Les Rites d'interaction. Éditions de Minuit, 1974. 


\section{Chapitre 1. Évolution historique : dix ans de débats à Beaubourg}

1 La production de débats au Centre Georges Pompidou est liée à sa vocation de diffusion culturelle. Elle subit cependant des variations d'ordre quantitatif et qualitatif au cours de la période analysée, 1978-1989. Ces variations sont dues aux changements internes changements de politique culturelle, de personnel à l'intérieur d'un service - et aux changements sociaux qui soumettent la programmation aux aléas de la demande et aux nouveaux rôles assignés aux institutions culturelles. L'évolution quantitative n'a pas de signification absolue. Elle n'est utile que pour faire des comparaisons, signaler des tendances, des ruptures, des changements d'orientation.

2 Le calcul du nombre de débats renvoie à leur classement. On peut ainsi distinguer trois périodes qui correspondent chacune à un certain type de fonction assigné aux débats. Une première période est celle de la mise en place des débats, aux débuts du Centre, où ceux-ci sont limités à certaines instances et ont une mission spécifique (1978-1982); il lui succédera une période de généralisation des débats, tant en fonction des instances que des thèmes (1982-1985); cette seconde période correspond à une augmentation quantitative du nombre des débats : l'utopie du Centre comme lieu d'immédiateté et de rencontre, portée par la première période, y rencontre son accomplissement mais aussi sa limite. C'est donc tout naturellement qu'à cette explosion succède une troisième période, de remise en ordre (1985-1989), caractérisée moins par une diminution du nombre que par une formalisation plus grande des débats et de leur programmation: les cycles se font plus nombreux, les fonctions de chaque instance apparaissent mieux définies. Bien entendu, une telle périodisation ne se laisse voir qu'après coup, et ne correspond pas à des changements explicites d'orientation. Mieux, il est loisible de trouver les différents types de débats à toutes les étapes. Il s'agit donc d'une évolution tendancielle, qui s'opère très graduellement (les dates retenues n'ont qu'une valeur indicative) et qui ne saurait être hypostasiée. 


\section{La période des commencements (1978-1982)}

3 Une première période est caractérisée par la mise en place des débats. La "Revue parlée », la BPI et le CCI sont trois instances productrices de manifestations orales, initiatives qui seront généralisées par la suite à d'autres structures du Centre. Rencontres, tables rondes, conférences se font en liaison avec les activités respectives de chacune d'elles. Mais c'est la « Revue parlée » qui incarne le mieux, au début, ce type de manifestations centrées sur l'expression de divers courants littéraires ou de pensée. L'idée de la "Revue parlée ", due à son concepteur et animateur Blaise Gautier, est en effet de reprendre les principales rubriques d'une revue littéraire en les adaptant à l'oral et à sa contemporanéité (l'importance du modèle de la revue est à la mesure de la place régulièrement accordée aux revues par la cellule de Blaise Gautier). Là où les lectures viennent tout naturellement prendre la place des textes littéraires de revue, les débats occupent celle des textes théoriques, de critique ou de pensée. Si l'idée de la « Revue parlée » est active dès la création du Centre, l'année 1977 ne présente toutefois pas d'archives exploitables, même si elle a laissé la trace de débats mémorables comme celui sur les « Nouveaux philosophes ». En outre, cette année de lancement du Centre ne se prête guère aux comparaisons. Aussi avons-nous commencé par l'année 1978.

4 Le schéma des vingt-cinq débats proposés, en 1978, par la « Revue parlée » permet de comprendre l'articulation des thèmes. De l'examen de cette année 1978 (cf. encadré), il ressort que les débats de la « Revue parlée » tournent essentiellement autour de deux axes, l'un qui est représenté par la littérature, l'autre par le champ de l'ethnologie. Bien que l'expression littéraire et poétique soit la ligne directrice qui identifie la Revue, la présence d'un champ de pensée est une constante sur plusieurs années, la philosophie alternant avec l'ethnologie. Les débats littéraires comme les débats de sciences humaines ont pour objectif de donner la parole à des auteurs, qu'il s'agisse de représentants d'une avant-garde ou de personnalités connues par leurs écrits et venant se confronter à un public. Un écrivain comme Pierre Guyotat illustre le thème d'une nouvelle problématique de l'écriture. Robert Jaulin est le représentant d'un courant ethnologique qui réintroduit la subjectivité dans l'enquête participante.

5 La place donnée aux littératures étrangères comme aux cultures minoritaires va dans le même sens d'assurer une visibilité à des objets culturels ou à des auteurs venus d'ailleurs. «L'écrivain latino-américain », «Les journées de la culture yiddish » en sont des exemples. La « Revue parlée » assure également une place aux médias en organisant une « Tribune littéraire » sur le thème : "Journalisme et littérature ». Elle se fait l'écho des autres activités du Centre avec la présentation de la revue Traverses, une table ronde autour de Bonhoeffer, renvoyant à l'exposition Paris-Berlin, la présentation du Diatope de Xénakis.

Une année de la « Revue parlée » 1978

Littérature française

Roger Caillois : « L'œuvre en train de se faire »

Pierre Guyotat : «La voix dans l'écriture : la découverte de la logique »

Jean Gillibert : « Délire et poésie »

Denis Roche

Littérature étrangère

Italo Calvino : « Lecture de villes » 


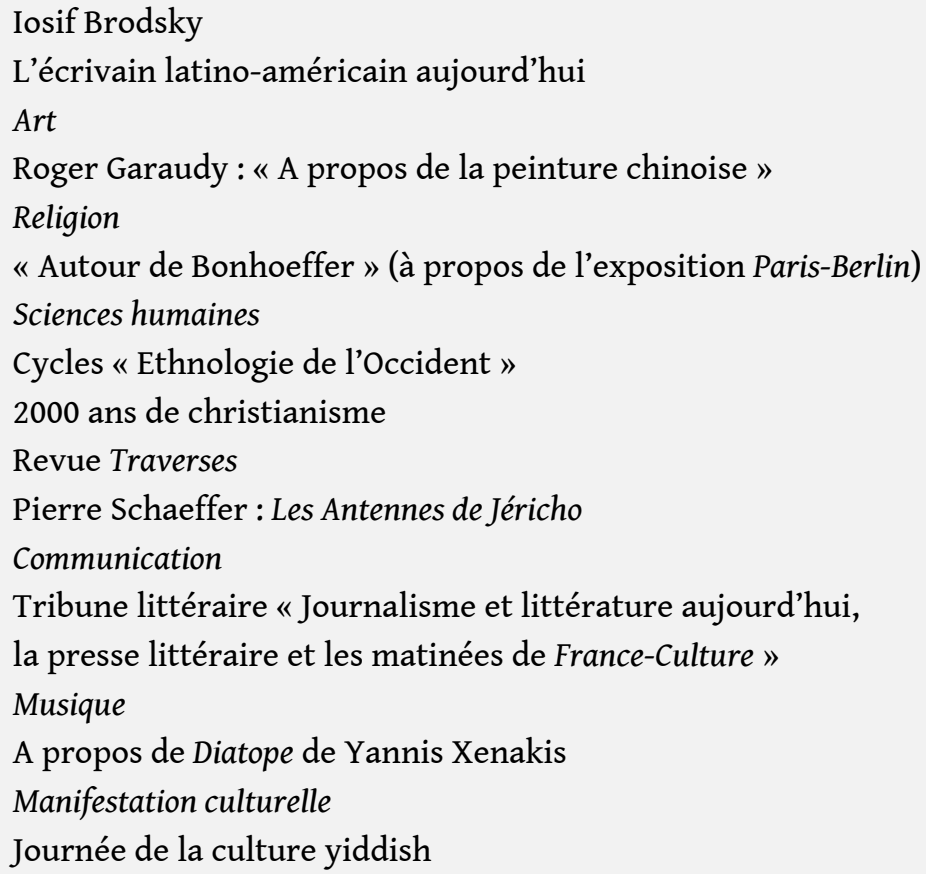

6 Un débat ouvre l'année 1978 avec Roger Caillois, dont l'encyclopédisme peut aisément être compris comme emblématique du projet de la "Revue parlée ", lui qui combine souci sociologique, expérience esthétique et réflexion critique. Elle se termine avec Yannis Xénakis. Le cycle «Ethnologie de l'Occident » s'étale sur cinq mois. La structure de la «Revue parlée » fait alterner les débats sur la littérature (complémentaires de lectures publiques) et les débats de sciences humaines. Elle donne ainsi la parole tantôt à des créateurs, des avant-gardes, des minoritaires, tantôt à des penseurs connus dans le champ médiatique. Elle ouvre enfin un espace où le Centre se pense lui-même, réfléchit sur sa création. Cette structure reste constante pendant la période considérée, qui va de la création du Centre à 1982 .

7 La BPI est la seconde instance productrice de débats. Les thématiques proposées sont aussi la création littéraire et artistique et les sciences humaines auxquelles s'ajoutent les problèmes de société et la communication. Cette plus grande diversification s'explique par le lien entre les débats dits de la "Salle d'actualité » et leur ouverture sur les nouveautés éditoriales. La Salle d'actualité est en relation avec un grand nombre de maisons d'édition (environ 300) dont elle reçoit les nouveautés en service de presse qu'elle affiche sur ses rayonnages ou par ses débats. A la différence d'une instance comme la "Revue parlée », la Salle d'actualité, qui est une sorte de chambre d'écho, a connu plusieurs types d'animateurs privilégiant différentes stratégies et thématiques. On peut ainsi distinguer trois styles et trois moments de la Salle d'actualité qui courent sur les dix années considérées mais qu'il est utile d'analyser en dehors de la périodisation annoncée dans la mesure où ils répondent à d'autres mécanismes.

Une première époque, selon celui qui en fut le principal animateur, Philippe Arbaizar, se situe "dans la mouvance de la lecture publique ». Il " organise », ou plutôt il suscite des rencontres avec des auteurs, au moment de la parution de leur livre. La conversation-rencontre, nom donné à ces manifestations, ressemblerait étrangement aux rencontres de la «Revue parlée». Mais dans un cas, un auteur vient parler de ses 
œuvres, alors que dans l'autre, il est rendu hommage à un auteur autour de ses œuvres (cf. p. 27).

9 En 1982, Philippe Arbaizar cède la place à Claire Stra qui, après un court intermède, sera remplacée par Bernard Falga. Celui-ci instituera un nouveau style de manifestation publique, plus proche de la confrontation d'idées autour d'un thème, tout en restant fidèle à sa vocation de promotion de livres. S'y adjoint une seconde vocation, plus universitaire, d'animation de débats pour un public que l'on espère fidéliser. La troisième époque, où l'on est encore, voit succéder à Bernard Falga une équipe de trois personnes et parait se caractériser, pour une part, par une plus grande délégation de la responsabilité des débats à des concepteurs extérieurs qui deviennent des collaborateurs réguliers (comme Claire Ambroselli), ou encore par l'enregistrement de propositions diverses, émanant aussi bien de la presse que d'associations. Les responsables des débats assument alors davantage un travail de coordination, sans négliger une activité spécifique de programmation, comme "Domaine privé» ou «Reportage photographique».

Pensé moins en fonction d'exigences internes au Centre qu'en fonction d'une actualité éditoriale a priori mouvante, le choix des débats de la Salle d'actualité de la BPI est le résultat d'un arbitrage entre une offre extérieure et une logique interne. Ce rôle de mise en forme d'une matière venue de l'extérieur et à laquelle chacun des différents responsables aurait donné son "empreinte » explique la diversité des thèmes retenus par la Salle d'actualité et qui tendent à l'exhaustivité. La première période aurait été plus littéraire. Mais le style de cette instance lui vient d'avoir ajouté très tôt, à la littérature et aux sciences humaines, les problèmes de société, représentés par des thèmes comme «Les femmes et la crise » (1978), « L'Afrique des inquiétudes » (1979), « La drogue » (1980).

11 Aux conversations-rencontres s'ajoutent les débats autour des problèmes de la communication et autour des expositions. Les premières sont présentes avec le "Carrefour des pratiques audio-visuelles régionales» et diverses présentations de collections scientifiques ou pour la jeunesse en 1978, avec un colloque de l'Unicef et deux cycles sur «Les médias et l'enfant» en 1979, par exemple. Les secondes sont organisées par le service de l'iconographie qui réalise les expositions de la BPI. Voici trois années de débats/expositions :

- en 1978 : une exposition Villes en images. Elle fait écho à celle du ccı La ville et l'enfant et les deux instances organisent un débat commun sur «Ville et natalité » en janvier 1978 ;

- en 1979 : six débats ont lieu autour de l'exposition Vu en Chine;

-en 1980: six débats sur Images de l'Amérique en crise, trois débats autour de l'exposition Volcans.

12 La structure des débats du CCI obéit à deux logiques : l'animation de ses manifestations qui analysent «les phénomènes de société dans leur relation à la production industrielle et à la production d'espaces »: ils sont le fait de la cellule "innovation sociale » ou de la cellule « innovation technologique». La production de conférences/ séminaires sur l'architecture et l'enseignement du design obéit à une seconde logique qui est de mettre en relation un public ciblé avec des professionnels reconnus, français ou étrangers. Le CCI fait alors appel à des organismes extérieurs dont la participation met le Centre dans un rôle de prestataire de service tout en consacrant son ouverture et sa crédibilité nationale et internationale. 
L'association «Architecture et construction » a ainsi organisé des conférences pour le Centre pendant plus de dix ans. Créée avec l'appui financier du ministère de l'Équipement, elle avait, à l'origine, pour vocation de réaliser des études techniques en collaboration avec le ministère. Progressivement, elle a étendu le champ de son objectif qui devint alors la diffusion de l'innovation architecturale, sous différentes formes : les études, mais aussi les expositions et les conférences. Ces dernières sont devenues un peu l'image de marque de l'association qui institua la tradition, au Centre Georges Pompidou, des conférences du mercredi (une par mois environ) à l'intention des étudiants d'architecture et des professionnels. Ils en constituaient le public privilégié et leur presse, le support de l'information préalable.

Il faut faire une place spéciale aux activités organisées par la Cellule liaison/adhésion qui a une politique de sensibilisation à la création artistique vis-à-vis des usagers potentiels de Beaubourg. Dans un premier temps, il s'agissait et de diffuser l'information sur le Centre et d'identifier des demandes tout en proposant un mode d'emploi de l'institution culturelle. La cellule s'est appuyée sur des relais culturels enseignants, délégués de comités d'entreprise - pour atteindre des publics variés et les amener à participer aux activités du Centre. Elle trouvait, dans ce public-relais appelé "correspondants", des partenaires motivés et parties prenantes du projet de Beaubourg. Ils furent les premiers destinataires d'une formation culturelle, conçue comme une initiation à l'art contemporain. Pour que l'institution culturelle gagnât un public, elle devait le préparer à pénétrer le sens de ses expositions ou de ses concerts. Mais devant le refus du MNAM de répondre à une demande de ce type, la cellule a fonctionné en parallèle. En même temps, la personne chargée des correspondants fut nommée responsable de l'animation au MNAM.

Les débats du $\mathrm{CCl}$

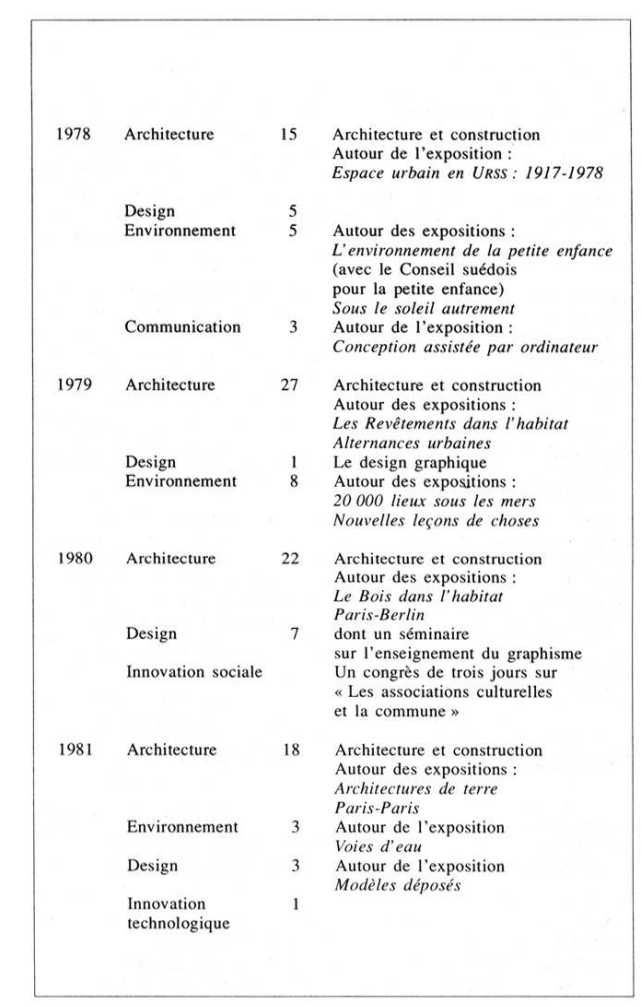


Les conférences-débats de la Cellule liaison/adhésion constituent donc une initiation aux principaux domaines culturels : arts plastiques, musique, architecture, littérature. Le tableau présenté en annexe sur les "réunions organisées par la Cellule liaison/ adhésion" montre que, dès le début, elle prévoit des rencontres sur le lieu des manifestations avec des artistes, des architectes ou des musiciens; elle propose des tables rondes-débats autour des grands événements du Centre. C'est ainsi qu'en 1981, elle organisa, autour de l'exposition Dali, un montage de textes du peintre lus par un comédien et commentés par Michel Ragon. Lecture critique qui s'opposait à l'attitude du MNAM face au public des œuvres d'art.

A partir de 1981, la cellule étend ses activités, jusque-là réservées aux seuls correspondants-relais, à l'ensemble des adhérents. Celles-ci peuvent être spécifiques et s'inscrire parallèlement à l'animation faite par le MNAM. Dans ce cas la cellule utilise les structures mises en place par les départements. Mais le privilège accordé par le MNAM à la recherche sur la vulgarisation, l'absence de cellule pédagogique au CCI et à l'IRCAM ont conduit la Cellule des adhérents à mettre en place des cycles de formation aux trois domaines principaux des arts plastiques, de la musique et de l'architecture contemporains.

\section{Généralisation des débats (1982-1985)}

17 Une deuxième période s'ouvre à partir de 1982 jusqu'à 1985 et correspond à une généralisation des débats. Ils concernent toutes les instances du Centre et des thèmes ou des questions de plus en plus variés.

Les chiffres globaux cependant n'indiquent pas de rupture mais montrent l'image d'un continuum avec une explosion en 1983. Dès 1980, la « Revue parlée » multiplie les débats sur la littérature, donnant la parole à davantage d'auteurs, davantage de revues, organisant un cycle sur les formalistes russes qui sera suivi par beaucoup d'autres: Ferrare, Panofsky, «Vienne, fin de siècle et modernité ». Elle donne plus d'ampleur également aux cultures minoritaires, après le succès des Journées sur les cultures juives méditerranéennes et orientales ( $c f$. chapitre 4$)$.

19 1983, c'est aussi l'année d'explosion des conférences-débats sur les problèmes de communication et les industries culturelles. La BPI en organise dix-sept en 1983, notamment deux colloques, dont un sur la télématique et un sur "Créativité et culture ». La "Revue parlée » accueille trois débats de la Maison du livre et des mots, en liaison avec le Carrefour des régions qui, du 19 mars au 26 avril 1983, propose par ailleurs vingt rencontres avec des maisons d'édition.

20 Mais c'est le MNAM qui peut servir de révélateur à l'expansion des débats. En 1978, il organise un seul débat, sur Malevitch, en liaison avec une exposition. En 1980, il organise six conférences sur des sujets variés, allant du commentaire sur des œuvres exposées à des sujets rejoignant la philosophie esthétique ou la muséologie.

21 A partir de 1981, une personne est nommée responsable de l'animation au MNAM. Cette année-là, l'ensemble des manifestations orales tripleront. C'est en 1983 qu'on assiste à leur explosion, le nombre des débats du musée rejoignant celui des autres départements, et dépassant même celui du cci. En créant une cellule d'animation, le MNAM ne cherche pas cependant à assurer une possible fonction pédagogique mais vise au contraire un public déjà averti. Il fonde sa stratégie sur la mise en place d'activités 
de réflexion et de recherche. Elles existeront désormais à côté des visites du musée accompagnées par des animateurs et des rencontres avec les artistes sur le lieu de l'exposition, qui constituent l'activité d'animation proprement dite. Le second niveau d'activité est celui des conférences débats destinées aux adhérents, à des étudiants et à des chercheurs, et qui soit portent sur le sens et le discours véhiculé par une exposition, soit posent des questions d'histoire de l'art ou d'esthétique. Ce second niveau institue une rupture entre le grand public, qui demande une initiation à l'art contemporain, et un public spécifique que l'on met en face de l'actualité de la critique et qui est considéré comme un public actif. L'aboutissement des conférences vers une production écrite - Les Cahiers du musée - les soumet à une exigence qui est celle du dialogue possible avec la recherche internationale.

Si on continue à prendre le MNAM comme référence d'une multiplication des lieux de parole au Centre, on s'aperçoit que 1983 représente bien un sommet. Le chiffre atteint par les débats du MNAM est le plus élevé de la décennie. Le chiffre total des débats amorce également une hausse ( $c f$. annexes).

Dans ce rôle nouveau, assuré par le MNAM et jusque-là réservé aux instances de production de sens, se lit donc l'importance grandissante prise par l'oral au Centre Georges Pompidou. A la diversification des lieux culturels (relais par le Centre, à l'instar des médias, des lieux traditionnels de la transmission culturelle) correspond une diversité des contenus. L'ampleur des débats du MNAM est emblématique de l'ampleur des débats du Centre. On assiste alors à une véritable mise à l'épreuve sociale des œuvres exposées. Une place grandissante est aussi donnée à la muséographie, en accord avec ce qui se fait ailleurs et avec la vocation d'ouverture du Centre qui le conduit à discourir sur ses propres pratiques. Cette dimension a été l'occasion d'accueillir de nombreux conservateurs français ou étrangers autour d'une réflexion sur le projet, trace écrite qui vient en amont d'une exposition et sur le paradoxe que constitue la conservation d'œuvres de création contemporaine. Le musée, comme l'écrit Michel de Certeau, n'est plus seulement le lieu du voir. Il ouvre beaucoup de questions sur l'expérience esthétique, «les relations que l'idéologique entretient avec l'esthétique et sur l'interpénétration de plusieurs types de perception ${ }^{1} »$. Avec l'existence des Cahiers, il met en scène ce qui fait aussi l'objet d'une série de recherches sur voir et lire.

\section{MNAM}

1981 : douze rencontres :

vingt-deux conférences autour des expositions :

Les Réalismes 1919-1939; Paris-Paris 1937-1957

quatre conférences hors exposition : muséographie

1982 : quatorze rencontres avec des artistes

treize conférences sur les expositions

neuf conférences hors exposition

«Sur l'art contemporain»

«L'art et les médias »

"Monteverdi : musique et mimétique »

1983 : quatre rencontres

trente-sept conférences dont vingt regroupées en trois cycles et une table ronde hors exposition

" Art international et expressions artistiques contemporaines du tiers monde » 
« Les dimensions du modernisme»

«Situation de l'art contemporain

à travers les grandes manifestations internationales »

« Fin des avant-gardes? » (table ronde)

24 BPI, lieu de l'écrit et de la parole, augmente le nombre de ses expositions. Elle emprunte à la «Revue parlée » l'idée des lectures publiques et accueille un festival de poésie. A partir de 1981, en effet, la BPI qui a toujours accueilli des expositions de photos ou de livres dans l'espace de la Salle d'actualité ou de la salle de lecture du second étage, organise chaque année plusieurs cycles de débats/commentaires.

En 1981 : les expositions commentées sont au nombre de quatre. C'est d'abord, au cours du premier trimestre, Côté jardins à partir de laquelle la BPI organise plusieurs projections-débats ou conférences; Robert Musil: deux débats. La Grèce au présent, exposition réalisée à l'occasion de l'entrée de la Grèce dans le Marché commun est accompagnée de cinq débats.

En 1982 : deux expositions sur la presse sont organisées, dont une de façon conjointe avec le ccI. La deuxième, Comment va la presse?, offre l'occasion de nombreuses rencontres avec des professionnels de l'information écrite et audiovisuelle ainsi que de débats ; quatre débats autour de l'exposition Des Repères pour l'homme ; trois débats autour de Lettres des Amériques.

En 1983 : un colloque en liaison avec une exposition Terres : Terre, création et pédagogie ; quatre débats sur l'exposition Fera-t-il beau demain? une conversation-rencontre commente l'exposition De la rosière à la miss.

En 1984 : Journée de l'Encyclopédie des sciences et techniques; quatre débats dans le Forum de l'édition photographique ; un débat sur Images à la page.

La " Revue parlée », elle aussi, utilise l'expression multi-médias autour d'un auteur ou des journées sur les cultures minoritaires ou lointaines. Après les journées des «Cultures juives méditerranéennes et orientales », viennent le Zaïre, la culture russe en France, le Chili - dont les manifestations sont autant visuelles qu'orales. En 1983, elle présente une exposition en collaboration avec le Centre régional de la chanson de Bourges : Têtes d'affiche.

30 En 1984, le CCI organise un grand nombre de rencontres autour de l'exposition Les Enfants de l'immigration. Elles ont été réalisées par la cellule « innovation sociale » que le responsable de l'époque, Paul Blanquart, désirait orienter vers le problème des rapports Nord-Sud. La cellule produisait une collection appelée «Culture au quotidien » et qui servait de support documentaire aux collectivités locales. Le ccI avait un rôle de conseiller auprès de ces dernières face aux problèmes d'aménagement et de relations à des populations spécifiques, souvent marginalisées. L'exposition sur les enfants de l'immigration était donc destinée à porter sur la place publique certaines images des rapports Nord-Sud telles qu'elles étaient exprimées par les populations immigrées.

La cellule " innovation sociale » avait aménagé une scène dans l'espace de l'exposition pour des animations quotidiennes. Radio beur organisa plusieurs débats dans cet espace ou dans celui de la petite salle. Avec cette manifestation qui utilise le concours d'une radio libre et d'associations d'immigrés, le ccI dont le nombre de débats reste, par 
ailleurs, à peu près constant, entre aussi dans une phase d'expansion des productions orales (dans les annexes, le chiffre porté dans la colonne " problèmes de société » pour l'année 1984 ne rend compte que des débats repérables dans les archives).

Le foisonnement des débats dans cette seconde période - et la dernière série citée en est un bon exemple-peut être considéré comme l'aboutissement de l'utopie du Centre. $\mathrm{Au}$ départ, fondée sur la croyance que la parole publique est créatrice de vérité, l'utopie favorise la confrontation d'idées. Dix ans après la remise en cause des structures de l'école, la contestation militante trouve son prolongement culturel dans les activités de débat proposées à Beaubourg. Puis l'idée d'une scène où s'exprime la parole publique dérive vers la multiplication des lieux de parole et des thèmes. Avec la fin des utopies qui correspond à la prise de conscience de leurs déviations, s'ouvre une période de reprise en main des débats.

\section{Mise en ordre des débats (1985-1989)}

Elle correspond à un double processus de spécialisation de chaque instance et d'organisation des rencontres qui tendront de plus en plus à prendre la forme de cycles. A l'éparpillement et à la multiplication inflationniste en réponse aux sollicitations extérieures - un débat/un thème, tout le monde parle sur tout - fait place une période d'organisation.

Avec l'augmentation des débats, les différentes instances de la BPI affirment leur spécificité, et se livrent même une certaine concurrence. On distingue très nettement, à la fin de la décennie, la Salle d'actualité, le service d'animation (dont le lieu privilégié d'expression est le forum) et le service de l'iconographie qui monte et anime des expositions. La première, que sa vocation conduit à enregistrer des initiatives extérieures de plus en plus nombreuses, réserve une place, cependant, à des formules plus organisées. Si elle continue à s'ouvrir sur les divers champs couverts par l'édition, elle accueille des groupes de réflexion selon une stratégie qui obéit davantage au cumul qu'à la mise en ordre. Etienne Balibar introduit le débat philosophique avec le « cabinet de lecture » (1984 à 1987) dont l'expérience sera poursuivie par le Collège international de philosophie. Le changement de tutelle signe le passage d'une structure ouverte et souple, fondée sur la critique des philosophes, à une structure plus fermée, qui dérive parfois vers le séminaire académique et peut privilégier un seul courant de pensée.

Le cycle «La fabrique du corps humain et les droits de l'homme " (1987-1988), issu d'une demande adressée par la Bpi, à l'Inserm, se trouvera placé sous l'égide de deux personnes (Claire Ambroselli et Antoine Spire), qui adoptent une forme souple mettant en présence plusieurs interlocuteurs : l'atelier de production d'idées.

\section{Cycles de débats à la BPI}

1985 : Colloque sur « La voix maintenant et ailleurs »

Colloque " Image et sciences"

Le mois du livre et de la lecture

Série de conversations-rencontres en collaboration avec Le Matin de Paris

1986 : Journée d'étude sur la critique des livres pour la jeunesse

Colloque national « L'enfant et la poésie »

Colloque Beckett

Le café viennois (neuf conférences-débats) et colloque Karl Kraus Débat sur 
«Images codées »

«Pleins feux sur les arts du spectacle» : sept débats.

1987 : Cycle « La fabrique du corps humain et les droits de l'homme » : huit débats

1988 : « La fabrique du corps humain et les droits de l'homme : neuf débats Cycle

sur l'écriture en rapport avec l'exposition

«Domaine privé » : neuf conférences

Exposition « La bibliothèque idéale » : quatre débats, en collaboration avec la

Maison des écrivains

Forum du reportage : quatre débats.

Le service " animation» de la BPI atteint la dimension d'une structure productrice de débats qui peut alors concurrencer les autres avec la création du " café viennois ». Mise en cycle de débats autour de l'exposition Vienne naissance d'un siècle, cette série inscrit au programme de cette reconstitution, et dans la tradition du café littéraire, la mise en scène du mouvement des idées qui ont marqué une époque. Dans le forum du Centre est reconstitué un café du début du siècle où l'on peut entendre, en consommant chocolat et gâteaux viennois, quelques interprètes des courants de pensée qui animèrent la Vienne du début du siècle :-L'ex-chancelier Bruno Kreisky inaugure la première soirée animée par Michel Cullin ;

- Jean-Louis Poitevin anime «La littérature autrichienne d'après guerre »;

- Michaël Löwy : « Les juifs et Vienne. Antisémitisme, sionisme et socialisme »;

- René Major : «Puissance des masses et genèse des despotismes »;

- René Lew : «Freud et Vienne »;

- Christine Buci-Glucksmann : «Vienne et l'obsession du féminin ».

Le cycle «Forum de la Révolution » (1989) aura lieu lui aussi dans l'enceinte du forum. Il est organisé avec le concours de l'équipe de Michel Vovelle et inaugure la formule technique de la radio et télévision-conférences, liaison en direct des orateurs invités par le Centre avec un membre d'un institut de recherche étranger. La thématique des débats est alors soumise au double critère de la programmation interne et de la rencontre avec l'extérieur.

L'encadré ci-contre présente les différentes séries de débats organisées par la BPI qui se sont déroulées de 1985 à 1988. On voit que la communication est très présente avec tous les cycles organisés par le service de l'iconographie autour d'expositions ou de journées qui consonnent avec l'activité de la bibliothèque.

A partir de 1985, la «Revue parlée » recentre également ses débats autour de deux thèmes majeurs: la littérature et les exotismes (l'ailleurs) qu'il s'agisse de présenter l'autre littéraire, social ou culturel. La grille de 1988 permet de voir l'évolution depuis 1978 (cf. encadré page suivante). Elle n'est pas marquée par la mise en ordre, la « Revue parlée » ayant toujours fait alterner les cycles et les manifestations plus ponctuelles. L'ouverture sur la scène internationale, dans les domaines de la littérature et de l'art principalement, a pris le pas sur l'avant-gardisme qui ne s'exprime guère qu'à travers l'œuvre de l'architecte Jacques Couëlle auquel le CCI consacre une exposition Vivre ailleurs aujourd'hui.

1985 est l'année du changement de cap des débats de l'association Architecture et construction. On passe de la structure un thème/un intervenant à une structure de deux cycles par an mettant en scène plusieurs intervenants de plusieurs disciplines : un architecte et un sociologue, un architecte et un cinéaste, un architecte et un peintre. 
L'architecture est passée en filigrane derrière un thème porteur. Citons l'exemple du cycle "La ville décline ses mémoires", au cours duquel un écrivain a fait une ode à Barcelone, un sémiologue a parlé d'Urbino à partir des tableaux et de la mémoire, Venise a été évoquée par un historien. La recherche de sujets nouveaux était soumise à la possibilité de leur articulation à d'autres disciplines du champ culturel, confirmant ainsi et accompagnant le mouvement qui devait progressivement faire de l'architecture une discipline-phare dans la réorganisation du champ intellectuel, notamment à travers les usages qui seront faits du thème de la post-modernité. En même temps, l'architecture se découvrait aussi tributaire de ces autres disciplines (cf. chapitre 4).

En 1985 est créée la cellule Espace-séminaire et en 1986 Histoire et société. Ces instances qui augmentent le phénomène de concurrence cadrent cependant avec la mise en ordre. Les débats qu'elles organisent sont peu nombreux et articulés autour de thématiques, philosophiques pour l'une, historiques pour l'autre.

Une année de la « Revue parlée » : 1988

Littérature française :

Hommage à Marcel Jouhandeau

«Pour Georges Perros »

Rencontre avec Eugène Ionesco

Revue Alphée

Littérature étrangère

Présence et texte dans le cadre des « Belles étrangères »:

- l'Argentine

- l'Espagne

- le Portugal

- la Chine

Hommage à Djuna Bames

Art

Autour de Mario Praz

L'Arène de l'art de Henri Cueco et Pierre Gaudibert

Berlin 1988, une image venue d'ailleurs

Journée Odysseus Elytis

Philosophie

Moïse ou la preuve par l'alphabet de l'existence de Yahvé de Jérôme Peignot

Revue TXT

Psychanalyse

" Art et Folie », présentation Jean Oury

La revue Chimères, présentation Félix Guattari

Sciences humaines

Le Temps des tribus de Michel Maffesoli

"A propos de la culture africaine "

Femmes du Maghreb

Architecture

Rencontre avec Jacques Couëlle

Manifestation culturelle

La Côte-d'Ivoire au quotidien 
Leur inscription dans l'institution correspond à son inflexion dans un sens davantage " universitaire ». Autrement dit, à côté des départements qui cherchent à préserver leur autonomie et leur hégémonie sur le Centre, ces nouvelles structures reliées directement à la présidence apparaissent comme marginales : mais elles témoignent aussi d'une modification notable dans la fonction et la perception du Centre. De lieu privilégié d'animation et de diffusion culturelle, il évolue vers une forme plus structurée de pédagogie culturelle, en se dotant d'une suite de « niveaux » progressive : les deux cellules ainsi évoquées fonctionnent un peu comme l'université du Centre. De fait, par le style de réflexion qu'elles impulsent et par les intervenants qu'elles sollicitent (une majeure partie d'universitaires), elles corroborent l'évolution en cycles de formation déjà constatée à propos d'autres instances. Nous reviendrons plus longuement dans la dernière partie sur la signification de cette évolution.

L'examen de dix ans de débats nous a révélé l'importance donnée à l'oralité dans un Centre où se manifeste, en apparence, "l'impérialisme du visuel ${ }^{2}$ ». Les débats augmentent en nombre, mais cette croissance quantitative n'a de sens que si elle est renvoyée aux phénomènes d'une diversification des champs culturels couverts et d'une généralisation à travers les départements. La demande interne et externe pour une production du discours, souvent liée à une production d'écrits, s'inscrit dans une évolution sociale qui voit se multiplier les lieux institutionnels de diffusion culturelle. Mais elle nécessite une programmation, loin des commencements utopiques, plus proche de l'institution universitaire.

44 La diversité des champs culturels couverts par les débats du Centre renvoie à une diversité des genres qui sera analysée dans la partie suivante. Cette diversité autorise la comparaison avec cette autre instance de diffusion culturelle qu'est France-Culture, qui à la différence de toute autre institution-musée, Fnac - ouvre son antenne à l'ensemble du champ. Le nombre des débats organisés par la chaîne de radio est cependant beaucoup plus important qu'à Beaubourg (cinq fois plus nombreux en 1980, la proportion est de trois pour un en 1988). France-Culture organise entre cinq et dix débats environ par jour, contre deux ou trois à Beaubourg. Dans une journée prise hors week-end, la chaîne propose un débat autour de l'actualité internationale qui sera suivi par Les chemins de la connaissance et Les matinées de France-Culture, deux séries, adoptant le style de la conversation et consacrées à des sujets historiques, scientifiques ou philosophiques. A l'heure du déjeuner, le Panorama présente des débats sur l'actualité éditoriale dont la Salle d'actualité a emprunté le style et les animateurs. L'après-midi offre, entre des lectures de textes et des concerts musicaux, un ou deux débats sur des thèmes artistiques ou littéraires. Entre 19 et $20 \mathrm{~h}$, deux émissions, Agora et Perspectives scientifiques, invitent des conférenciers. Le début de la soirée est réservé aux dramatiques ou aux reportages, plus rarement à une table ronde sur un problème de société. La soirée s'achève par "Les nuits magnétiques » qui offrent dans certains cas l'écoute de tables rondes.

45 Ce qui est à noter, c'est tout d'abord la grande stabilité de la chaîne radiophonique dont les émissions citées constituent un invariant, rythmant les journées au cours de la période étudiée. D’autre part, apparaît la vocation prédominante de France-Culture à diffuser des commentaires sur l'actualité ou la production littéraire, artistique ou scientifique. La radio remplit ainsi un rôle de formation para-universitaire qui la distingue du Centre Georges Pompidou notamment par l'ampleur des débats diffusés. Cette disproportion rend malaisée la comparaison. Mais nous avons essayé, à travers 
deux années de référence, 1980 et 1988, de suivre la fréquence relative des thématiques abordées. Seule la philosophie et les problèmes de société présentent un rapport équivalent au Centre et à France-Culture où ils ne sont pas prédominants. C'est le cas, en revanche, des débats sur la science, l'histoire, les sciences humaines, la littérature qui sont très représentés sur la chaîne radiophonique; elle est relativement moins présente que le Centre sur la scène de l'art, de l'architecture, de l'enseignement et des communications. Ces domaines représentent respectivement les trois principaux départements du Centre que sont le MNAM, le cCI et la BPI.

\section{NOTES}

1. Michel de Certeau, « Le sabbat encyclopédique du voir », op. cit.

2. Michel de Certeau, « Le sabbat encyclopédique du voir », op. cit., p. 66-83. 


\section{Chapitre 2. Les thématiques et les styles de débats : du spectacle à la conférence magistrale}

Le terme de débat est ici employé de façon générique; mais il recouvre une variété d'activités orales qui vont de la « rencontre autour »... d'un livre, d'un auteur, d'un événement artistique, social ou politique à la conférence classique où l'intervenant possédant une compétence légitimée s'adresse à un public. Entre ces deux types de face à face - le premier informel, le second plus structuré - entre un ou des intervenants et un public, existe tout un éventail de productions orales que nous avons regroupées dans des catégories qui les analysent selon deux axes principaux. Le premier est celui de l'actualité : un débat peut être lié à l'actualité artistique, médiatique, éditoriale, politique, etc., voire imposé par elle ou au contraire chercher délibérément à s'en affranchir. Le second axe est celui de la connaissance, en fonction de la volonté, manifeste ou non, de transmettre ou même de produire des connaissances, ou au contraire de présenter des œuvres ou de confronter des opinions. Ces deux axes partagent les débats qui apportent des témoignages et ceux qui apportent des connaissances d'une part; les débats structurés et les débats informels, d'autre part. 


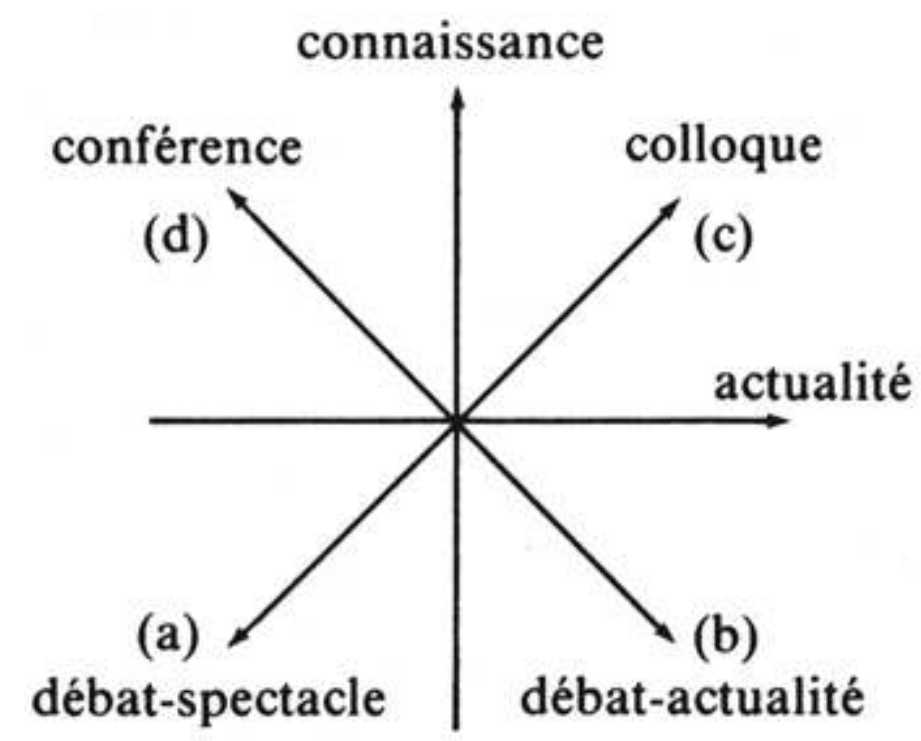

Si l'on s'avise maintenant de croiser les deux axes, on obtient un schéma qui distribue les débats en quatre types :

faible actualité - faible teneur en connaissance (a);

forte actualité - faible teneur en connaissance (b) ;

forte actualité - forte teneur en connaissance (c) ;

faible actualité - forte teneur en connaissance (d).

A chacun correspond un modèle que l'on trouve sous une forme plus ou moins pure dans les débats

effectivement pratiqués au Centre. Nous les avons appelés respectivement :

(a) les débats-spectacles

(b) les débats-actualités

(c) les débats-colloques

(d) les conférences.

2 Pour aboutir à cette modélisation des débats, les critères de classification combinent trois types de données :

- les discours des concepteurs sur les objectifs qu'ils ont donnés à ces débats et les représentations qu'en ont les divers intervenants ;

- les archives qui permettent d'opérer un premier repérage des genres selon l'axe actualité/ connaissance en fonction du lien de ces débats avec une actualité éditoriale, artistique ou politique et la position des intervenants dans le champ culturel ;

- l'étude ethnographique des débats, enfin, qui s'est attachée à leur mise en scène et aux pratiques de l'échange verbal, constituant un véritable « ordre rituel ».

3 Pour ce troisième mode d'approche, il est nécessaire de préciser au-delà de ce qui a été dit dans l'introduction ( $c f$. p. 6), les niveaux où se situe l'observation.

La mise en scène du débat est fonction des lieux. Elle est aussi fonction des médiations qui introduisent les acteurs de la rencontre. Les différents lieux n'ont pas la même capacité d'accueil. Ils n'ont pas non plus la même charge symbolique. Certains sont banalisés, comme la Salle d'actualité ou la salle Renoir. Les intervenants sont à hauteur $\mathrm{du}$ public, parfois assis en arc de cercle, faisant salon. Les salles du sous-sol sont des lieux théâtraux qui ont leurs coulisses, leur scène surélevée, des gradins, des effets d'éclairage. Le forum est le lieu qui symbolise le mieux l'utopie du Centre : lieu ouvert, au public mouvant, allant et venant au gré des flux d'entrée.

5 Les niveaux de médiation qui règlent l'entrée en scène des protagonistes permettent aussi d'opérer une différenciation des débats. Le face à face entre les acteurs et leur public peut se faire immédiatement, après un court préambule du président de séance 
annonçant le thème et l'ordre des interventions. Mais, le plus souvent, il ne peut se produire qu'après une série de présentations inaugurales, équivalentes à des salutations de bienvenue ou à des ouvertures solennelles. Cet ordre rituel, qui institue une certaine rigueur, fixe également le cadre dans lequel doit se dérouler l'échange. Les médiateurs qui appartiennent à la direction du Centre accueillent les invités tout en les initiant aux règles de la maison et à celles de la rencontre du jour. Il appartiendra au modérateur de poser les règles de l'échange verbal.

6 La forme et le nombre des médiations qui règlent l'entrée en scène sont fonction de la solennité de la rencontre - manifestation prestigieuse ou banale, première ou débat routinier - de la théâtralité qu'on veut lui donner ou encore de sa place dans les productions du Centre. Quand ce dernier n'est pas producteur de débats mais prestataire de service, c'est alors la place de la rencontre dans le champ culturel ou le champ de l'actualité qui détermine l'importance de la mise en scène.

7 Après l'ouverture, suit la présentation proprement dite de la rencontre par l'animateur. Il énonce les règles de procédure qui présideront à l'échange : accord sur le thème du débat, moments de la prise de parole de chacun des intervenants en fonction de son identité, présentation des intervenants. L'animateur propose les règles de la prise de parole. C'est dans la façon dont elles sont respectées, déjouées ou transgressées que se lisent les différents types de débats. Une étude des interactions verbales permet d'analyser le système des pratiques qui organisent la communication.

L'ordre des prises de parole est dicté formellement par l'animateur. Mais il est aussi déterminé par le genre du débat. L'animateur intervient avant chaque prise de parole. Son rôle est d'inviter les interlocuteurs à parler, légitimant leur participation. Il existe plusieurs types d'animation qui sont liés à la nature des débats : caractère périodique ou épisodique ; production interne ou initiative extérieure. L'animation "présidence " est la moins implicante et la plus fonctionnelle. Le rôle d'un président de séance est honorifique et se réduit à la distribution de la parole. Ce type d'animation est plutôt associé aux manifestations épisodiques : colloques structurés autour d'une succession de conférences. Le modérateur est plus engagé. Son rôle est plutôt associé à des rencontres périodiques ou à des cycles.

9 Le temps de parole donné au public est fixé d'avance ainsi que les règles de son entrée en scène. L'enquête ne portait pas sur les publics. La présence d'une audience a cependant un impact sur la mise en scène des débats, dans la façon dont elle est invitée à participer, mais aussi dans la façon dont elle manifeste, dans sa passivité, son approbation, sa connivence ou sa contestation.

\section{Analyse des modèles : le débat-spectacle}

C'est un premier type de débat qui ne doit rien à l'actualité mais qui est choisi en fonction du goût du programmateur. Il a également une faible finalité pédagogique et privilégie la rencontre. Les thèmes choisis reflètent deux dimensions du Centre qui sont la contemporanéité et l'internationalisme. La présentation de ces rencontres est magistrale et met en scène le côté spectaculaire de la parole. La plupart des débats de la «Revue parlée " peuvent entrer dans ce modèle. Première instance organisatrice de débats, avec la BPI, la « Revue parlée » a pour fonction essentielle de mettre en scène des productions littéraires - lectures publiques - ou des discours sur ces productions littéraires ou d'autres types de productions culturelles rencontres autour d'œuvres en 
présence ou non de leur(s) auteur(s). Une revue orale, à l'instar de son équivalent écrit, présente, au sens premier du terme, une série d'auteurs et de textes choisis en fonction d'un certain éclectisme et d'une résonance avec le lieu d'accueil. Les débats que nous avons appelés "débats-spectacles» mettent en présence du public plusieurs types d'auteurs et de textes.

\section{Des auteurs ou des textes individualisés}

Qu'il s'agisse d'avant-gardes littéraires ou de représentants de mouvements de pensée, leur production est achevée et accessible sous forme de textes. Les premières sont représentées par les groupes qui travaillent autour de l'écriture, les revues poétiques, parmi lesquelles citons Pictura Edelweiss, Logomotives, Babylone, Alea, Tarte à la crème, Textuerre...

12 Les seconds sont entrés à la «Revue parlée » avec la série "La philosophie aujourd'hui» qui a réuni en 1977 le groupe des «Nouveaux philosophes» sous la direction de Blaise Gautier et Jean-Marie Benoist. «Pourquoi philosopher encore?", animé par Christian Delacampagne, est une série plus récente qui succède au «Jardin des sciences ".

13 La philosophie ne s'inscrit pas dans la «Revue » sous la forme du séminaire mais de "l'ouverture sur l'instance du débat philosophique ». Il s'agit de mettre en scène, au sens de mettre sur la scène et de mettre en situation, des discours philosophiques multiples qui vont se croiser et s'articuler devant le public. La confrontation est convenue, préparée, plutôt que produite par la rencontre. On connaît à l'avance - le public comme les intervenants - les positions de chacun. Mais la scène produit de l'échange en acte.

14 La "Revue parlée ", qui offre aussi des débats plus centrés sur la transmission d'information (cf. p. 42) privilégie, dans ses rubriques "débats spectacles», des domaines non classiques. L'ethnologie et la psychanalyse côtoient ainsi la philosophie au titre de l'ouverture sur des champs de pensée qui ont conquis récemment leurs lettres de noblesse et qui représentent aux yeux d'un large public le monde contemporain et l'ailleurs, ce qui est à la fois de notre temps et producteur de nouvelles références.

Si le champ littéraire avant-gardiste offre l'occasion de découvrir de jeunes auteurs, les autres champs sont occupés par des personnalités légitimées dans la sphère universitaire ou éditoriale mais dont c'est la présence dans l'espace public qui crée l'événement. Ainsi, les débats de la "Revue parlée » entrent dans la catégorie de l'hommage au grand écrivain qui utilise le rituel de la célébration. L'hommage développe, par l'exposé des œuvres ou sur les œuvres, « une forme d'exemplarité ${ }^{1}$ ». Il a donc, à la fois, un souci pédagogique et la fonction d'un palmarès. Les débats qui rendent hommage adoptent une forme qui est toujours un peu convenue et leur intérêt réside alors non dans les thèmes abordés mais dans le plaisir d'assister à des joutes oratoires ou de voir sur scène le représentant d'un domaine qui reste habituellement fermé aux non-spécialistes. 


\section{Des groupes dont les productions sont en voie de constitution ou de légitimation} des individus parlant pour eux-mêmes mais des collectifs communautaires représentant Tailleurs culturel ou social. Le débat n'est qu'un mode d'approche d'une culture qui est présentée par des moyens multi-médias, alliant l'exposition d'œuvres et d'objets, l'expression audiovisuelle et l'expression orale : parole et chant. Les premières journées consacrées à une autre culture furent celles de la «culture yiddish» dont le succès donna à certains membres des autres communautés juives qui ne se reconnaissaient ni dans la culture ashkénaze, ni dans la culture française l'idée de se produire sur la place publique ( $c f$. chap. 4). Ce furent les journées des « cultures juives méditerranéennes et orientales ». Elles furent suivies par beaucoup d'autres : la culture russe en France (1982), les cultures africaines (1982), une « soirée minorités ethniques : Mapuches » dans le cadre de la manifestation "Chili, lorsque l'espoir s'exprime " (1983), auxquelles on peut ajouter les manifestations culturelles suivantes: "Voix Slovènes » (1986), «L'Europe centrale et les pays danubiens : réflexion contemporaine sur un phénomène culturel " (1987), "Côte-d'Ivoire au quotidien" (1988) qui sont autant d'occasions d'aller à la rencontre d'une culture.

Les collectifs invités sont aussi des mouvements représentatifs de l'autre social, dans ses modes d'expression les plus éclatés, les plus disparates. Citons la présentation d'«Art et folies » par la revue Chimères née de l'expérience de La Borde.

\section{Les auteurs étrangers}

Ils sont invités pour présenter les productions qui paraissent sur la scène internationale. La littérature étrangère est très présente au Centre Georges Pompidou qui joue le rôle d'amplificateur des activités des différents instituts culturels étrangers. Toujours associées à des lectures de textes, ces manifestations autour de la littérature venue d'ailleurs ne sont pas des « discours sur» mais donnent la parole aux auteurs. Elles existent depuis le début du Centre, organisées avec le concours de l'association Dialogue entre les cultures. Leur forme la plus achevée est l'opération «Les belles étrangères " qui se déroule simultanément dans plusieurs institutions de Paris et de province sous l'égide de la Direction du livre et de la lecture du ministère de la Culture et de l'association citée plus haut. Parmi ces lieux, dont les plus représentatifs sont la Sorbonne et la Fnac, le Centre occupe une place importante, pour les organisateurs, entre la séance inaugurale et le débat social. Il est le lieu de la rencontre entre les auteurs et les lecteurs potentiels.

Si la fonction de tous ces types de débats-spectacles est de donner la parole à des auteurs, des penseurs, des collectifs, leur finalité est dans leur existence même. Il s'agit d'exploiter le côté spectaculaire de la parole, la « philosophie » de tels débats reposant sur la croyance en l'oralité comme dimension de la communication culturelle à côté des arts acoustiques ou visuels.

Le titre qui les exprime le mieux est le suivant : «Autour de José Bergamin », «Autour de l'œuvre de Georges Dumézil». On retrouve la forme de l'hommage. Si la Salle d'actualité confère une légitimité aux auteurs qu'elle invite - selon un rituel qui est proche de celui des émissions littéraires de la télévision (Apostrophes) -, la « Revue 
parlée " s'apparente davantage à l'éloge du génie déjà reconnu ou passé. La comparaison pourrait être faite avec les émissions spéciales que B. Pivot consacre à un auteur de marque, interrogé sur sa vie et sur l'ensemble de son œuvre. Il s'agit de la forme laïcisée de l'oraison funèbre. Selon Jean-Claude Bonnet, l'audiovisuel qui rend hommage ressemble à une "mise en scène testamentaire et à une scénographie funèbre, comme si le grand vivant n'était déjà plus là que pour une adresse à la postérité ${ }^{~ » . ~ C ' e s t ~ a i n s i ~ q u e ~ l ' e ́ m i s s i o n ~ c o n s a c r e ́ e ~ a ̀ ~ G e o r g e s ~ D u m e ́ z i l ~ f u t ~ r e d i f f u s e ́ e, ~}$ quelque temps après sa mort, en hommage au grand penseur.

L'analyse ethnographique - mise en scène, étude des interactions verbales permettant d'étudier le système des pratiques qui organisent la communication, révèle bien leur double appartenance à des manifestations rencontres sans médiation solennelle, mais comportant un rituel théâtral. Les débats de la "Revue parlée " illustrent la forme du débat-spectacle avec effets de théâtralisation. Il y a un simulacre de lever de rideau, quand le directeur de la Revue, Blaise Gautier, annonce « Mesdames, messieurs... La "Revue parlée"! ». Il ne se tient pas sur la scène, avec ses invités, mais de côté. Il joue le rôle du récitant; introduit les protagonistes, modérateur et intervenants. Ensuite, c'est le modérateur qui entre en scène. Il introduit à son tour chaque prise de parole. Son rôle est d'inviter les interlocuteurs à parler, légitimant leur participation à discourir sur un auteur, à commenter son œuvre. L'accréditation peut être amplifiée, renforçant l'aspect spectaculaire du débat. L'interlocuteur-vedette est alors loué pour son savoir : «notre spécialiste », « le plus autorisé d'entre nous ». On se trouve devant ce que les linguistes appellent des "actes illocutoires ${ }^{3}$ ", donnant au locuteur l'autorisation de parler comme il parle. Quand l'accréditation de l'intervenant est moins forte, il en vient alors à s'excuser de prendre la parole. Jean Lacouture, lors de la rencontre " Pour José Bergamin ", invité à témoigner plus qu'à commenter, prend ainsi la parole: "Moi, le seul non hispanisant, je voudrais faire quelques observations... » Georges Auclair, remplaçant Patrick Reumaux dans le débat autour de l'ouvrage Le Temps des tribus commence ainsi son commentaire: "Après l'exposé de Michel Maffesoli, je suis un peu embarrassé. Je voudrais simplement dire... » Dans les débats-spectacles, s'il y a des vedettes, il y a aussi des seconds rôles.

Ce qui caractérise le débat-spectacle, c'est précisément le rôle que jouent les protagonistes dans le déroulement de la rencontre. L'ordre des prises de parole est dicté non par la logique du débat mais par l'identité des intervenants. Dans la « Revue parlée ", cet ordre correspond à un mélange des genres : le sociologue et l'écrivain; le journaliste et le critique littéraire interviennent tour à tour pour faire le commentaire d'une œuvre. Le résultat s'apparente davantage à des variations musicales sur un même thème qu'à un débat d'idées, même si certains intervenants se piquent au jeu de la controverse. Mais Blaise Gautier est là pour rétablir l'ordre convenu, qui fait se succéder plusieurs lectures d'un même texte. L'appartenance de l'intervenant à un champ ou à un autre fonde sa légitimité à parler à tel moment et de telle façon. Jean Lacouture, invité à remémorer ses rencontres avec José Bergamin, reçoit un rôle de témoin, moins important que les rôles de critiques littéraires qu'avaient eus avant lui deux spécialistes de la tauromachie. En lançant la controverse autour de la traduction de l'œuvre de Bergamin, pour « animer le débat », il reprend le rôle de journaliste qu'il tient sur d'autres scènes. 


\section{Le débat-actualité}

23 Une deuxième forme de débats correspond à la rencontre d'un ou plusieurs intervenants avec le public autour d'un thème d'actualité. Il peut s'agir d'un événement éditorial, politique ou artistique. Le modèle exemplaire en est la conversationrencontre organisée par la BPI dans la Salle d'actualité. Les sujets de débats sont alors étroitement liés à la vocation de la salle qui est de rendre compte de l'actualité éditoriale. Il en résulte un éclectisme des thèmes puisés dans différents domaines: littérature, sciences humaines, enseignement, sciences. A cette actualité purement éditoriale vient s'ajouter l'actualité politique ou sociale qui est traitée dans les débats que nous avons appelés "problèmes de société » mais qui peuvent aussi être abordés sous un angle sociologique ou philosophique.

La succession des débats hebdomadaires fait donc écho à la succession des rayons d'une librairie et à celle des grands bouleversements sociopolitiques; la logique est factuelle, le choix résultant d'une combinaison entre plusieurs facteurs : thèmes porteurs, choix qui ressort des organisateurs du Centre; succès des ouvrages dépendant du travail des attachées de presse ; personnalité des auteurs ou participants.

Les modèles qui ont sous-tendu ces conversations-rencontres - selon leurs responsables - ce sont les débats de la Fnac et ceux d'Apostrophes, la célèbre émission d'Antenne 2. Il s'agissait de remplir un plateau avec des auteurs ou des intervenants venus témoigner de nouveautés éditoriales. Le débat naît de la confrontation d'opinions entre les invités du plateau et du travail de l'animateur qui est alors souvent un journaliste, ayant une compétence de communication pour mener la discussion. Mais à la différence de ce qui se passe à la télévision, une troisième instance intervient qui est le public. Ce dernier n'est pas forcément partie prenante du débat. Mais il en est la raison d'être. Il est le destinataire de la confrontation et dans la période des commencements, période de l'utopie où l'échange de parole était considéré comme créateur, le public se trouvait transporté, par le débat, au cœur de la culture livresque, comme sa visite du musée le transportait au cœur de l'art contemporain. Le public rencontrait les intervenants, comme fortuitement, puisqu'au départ, la Salle d'actualité ne possédait pas de lieu réservé, clos et insonorisé. L'animation se passait sur le lieu de l'événement, au milieu des rayonnages présentant les dernières parutions, des lecteurs et des promeneurs.

Dans l'idéologie qui préside à la création de Beaubourg, l'accès à la culture est conçu comme spontané, sans médiation. Il est favorisé par l'architecture du lieu où domine la transparence. A cette visibilité sans médiation de la culture renvoie l'immédiateté du débat qui ne demande pas de préparation, puisque ce serait précisément de la confrontation de paroles que naîtrait la vérité.

L'analyse ethnographique des débats confirme cet aspect non médiatisé du débatactualité. Le lieu de sa mise en scène, tout d'abord, est banalisé. Les intervenants sont à hauteur du public, parfois assis en arc de cercle, faisant salon. Le face à face avec le public se fait presque immédiatement après une brève présentation du thème du débat et de ses participants. "C'est une réunion entre amis, moi excepté puisque j'en suis l'animateur", annonce ce dernier, d'entrée de jeu, en précisant que faute de contradicteur autour de la table, "nous allons chercher la contradiction, je l'espère, dans la salle qui est devant nous " (conversation-rencontre, "La Goutte-d'Or : histoire et actualité », septembre 1988). L'animateur, fidèle ici au modèle du débat spontané, 
souhaite, mais sans trop y croire, trouver dans la salle des personnes qui pourront décrire une expérience vécue, différente de l'analyse que font les auteurs du livre commenté. En fait, se présentera, en fin de séance, un nouvel «ami » retardataire que l'animateur s'empressera d'intégrer autour de la table.

Dans le débat-actualité, le rituel de présentation existe mais il se donne comme spontané. On laisse à chaque participant le soin de se présenter et d'annoncer son thème d'intervention. On joue l'intimité, révélant son plaisir à partager la scène avec telle personnalité. Le rituel de présentation peut alors être détourné au profit d'un jeu de la reconnaissance mutuelle entre maîtres et disciples, ou entre pairs dont certains peuvent être identifiés dans la salle. Le débat "Citoyenneté et nation" illustre le premier cas. Etienne Balibar prend la parole, au début de la conversation-rencontre, pour dire son émotion à être assis à côté d'Henri Lefebvre et de voir la reparution/ réapparition du Nationalisme contre les nations. En soulignant l'actualité d'un livre publié en 1937 et qui disparut aussitôt après, il légitime à la fois l'existence du débat - moment de célébration - et la présence conjointe des invités - Immanuel Wallerstein, Yves Lacoste et lui-même - dont les réflexions appellent la confrontation avec la pensée d'Henri Lefebvre.

L'ordre des prises de parole est dicté formellement par l'animateur/modérateur. Mais comme dans la conversation ordinaire, «il n'y a pas de temps mort, pas de discipline hiérarchique de la parole ${ }^{4}$. Si Étienne Balibar, qui est intervenant à la place de l'animateur, donne la parole en premier à Henri Lefebvre, c'est au nom de l'antériorité de sa pensée. Il se défend de faire un point d'ordre.

30 La conversation est marquée par un double mouvement de territorialisation et de prise de distance de la part des protagonistes ${ }^{5}$ (Ils doivent afficher leur position dans le champ qui est le leur. Mais ils doivent le faire avec réserve, sans exhibition, sans vouloir délivrer un message. La distance garantit une certaine auto-régulation des débats. Cependant, le débat entre des auteurs fonctionne comme lieu de la reconnaissance sociale. Dans les débats où l'enjeu porte davantage sur la légitimation des invités que sur le thème, il arrive que l'ordre se fasse désordre et que les intervenants prennent la parole au mépris de leur tour et des règles implicites de la conversation. Ce fut le cas d'un débat sur "L'humour juif » où l'animateur perdit le contrôle de la tribune et où chacun essayant d'imposer son histoire juive, la séance prit l'allure d'un concours. La transcription du débat révèle le nombre important des interactions verbales. On joue à la conversation entre amis, sautant d'un sujet à l'autre, prenant la parole à tort et à travers. Mais on n'ignore pas qu'on est sur une scène publique et que l'enjeu est de paraître.

31 L'animateur est souvent engagé. Il peut stimuler la discussion, l'orienter. Il est souvent un professionnel qui vient de la radio ou de la télévision et qui n'a pas de compétence particulière sur le thème du débat, mais il a une compétence de communication qui lui permet de jouer les intermédiaires entre les intervenants et le public.

Il faut cependant apporter quelques nuances à cette forme exemplaire du débat d'actualité. Elle comporte des variantes qui tiennent aux instances organisatrices et à l'évolution du Centre dans le temps. Au départ et en résonance avec l'utopie Beaubourg - lieu panoptique, ouvert, où circule la culture - les débats étaient conçus comme une animation liée aux missions spécifiques de chacun des départements. La mission principale de la bibliothèque est la lecture. On anime la salle de lecture en faisant se rencontrer des auteurs et des lecteurs potentiels. La mission du musée est de faire voir 
des œuvres d'art. On crée des animations-rencontres avec les artistes. Les organisateurs ne les conçoivent pas comme des formes de transmission pédagogique mais comme des dialogues, prolongement de l'accès à l'art contemporain dans la mesure où ce dernier appelle la parole de l'artiste. Le public peut donc interroger l'œuvre d'art à travers son créateur. Ce qu'il reçoit n'est pas de l'ordre de l'explication, ni du commentaire, mais participe de l'accès direct à la création.

Le CCI se situe entre la BPI et le MNAM. Il organise aussi bien des débats centrés sur l'actualité de l'édition (dans les domaines de l'architecture ou du design) que des débats liés à des expositions. Aux deux pôles de l'éventail thématique proposé, citons les rencontres autour de l'exposition "Conception assistée par ordinateur » en 1978 et qui met le public en présence des professionnels de la recherche informatique; les rencontres autour de l'exposition "Les enfants de l'immigration" en 1984 qui font intervenir diverses institutions ou responsables d'associations. Les deux sujets sont d'actualité. Le premier donne lieu à une abondante répercussion dans la presse. Le choix du second, au contraire, est l'effet de son actualité. Le Centre Georges Pompidou jouera cependant un rôle moteur dans la mobilisation des associations de défense immigrées en portant sur la scène publique la confrontation entre les responsables institutionnels, d'une part, les jeunes concernés, de l'autre.

La fin des utopies entraîne l'évolution du Centre (cf. chapitre 1) et fait dériver le débatactualité de la controverse à la juxtaposition de points de vue. Privées d'enjeu, les rencontres deviennent simples tours de table mais paradoxalement ou conséquemment leur nombre augmente. En effet, il suffit, pour trouver un thème de discussion de réunir plusieurs auteurs et la finalité du débat se déplace vers l'extérieur du Centre, qui dès lors assure la promotion des nouveautés éditoriales. Pour rompre avec l'effet de routine créé par la réponse aux sollicitations extérieures, la BPI a essayé de choisir des sujets chocs (la drogue, l'immigration, les droits de l'homme), de donner la parole à des associations qui parallèlement proposent des expositions en Salle d'actualité.

Une deuxième solution adoptée par les instances du Centre, et que nous avions soulignée comme mise en ordre, est de programmer des séries de débats sur un thème. La mise en ordre se traduit par deux types de transformation du débat-actualité : il évolue vers l'expérimentation sociale conservant une de ses anciennes dimensions, l'immédiateté, ou vers le colloque classé dans le troisième type de débats. Les séries sur "La fabrique du corps humain et les droits de l'homme", "L'atelier de droit international » organisées par la BPI et desquelles on peut rapprocher la série sur « Les enfants de l'immigration ", produite par le CCI, ne sont pas conçues comme de simples échanges d'idées. Elles aboutissent à des productions nées de la rencontre et pendant le déroulement de la rencontre. Le public participe à ces productions, que son rôle soit conçu comme une source d'informations que ne possèdent pas les intervenants officiels, ou qu'il s'agisse d'un "faux public ", les intervenants étant disséminés autour de la table et dans la salle pour l'animer. Le rapport au public reste comme dans l'idéaltype un rapport non médiatisé. Sa participation se veut plus effective, le spontanéisme des débuts utopiques évoluant vers l'idée qu'un travail intellectuel se construit à partir d'apports d'expériences. Le Centre Pompidou devient laboratoire social quand il organise autour de l'actualité politique ou sociale une production orale, débouchant parfois sur une forme écrite. De la série sur «La fabrique du corps humain » est né l'ouvrage Éthique médicale et droits de l'homme, publié chez Actes Sud. 


\section{Le débat-colloque}

Le type de débat actuellement le plus répandu, parce qu'il est à la fois le résultat d'une évolution et qu'il cadre avec les missions de toutes les instances du Centre, c'est le débat qui combine les exigences de l'actualité et de la transmission de savoir. Il est plus organisé au sens où il ne peut s'apparenter à la simple rencontre, au simple face à face avec le public. Il suppose une préparation qui va influer sur la forme définitive, toujours plus solennelle, s'apparentant au colloque qui débouche parfois sur l'écrit. Le débat-colloque comporte cependant plusieurs variantes en fonction des thèmes et des finalités qu'il se donne.

\section{La mise en cycle de type universitaire}

Il s'agit de la mise en série de thèmes d'actualité qui vont être traités par des spécialistes, le plus souvent issus du monde universitaire. Avec la forme du cycle intervient la mise à distance de l'actualité qui permet d'en rendre compte, de la rendre publique sur un mode savant, rompant avec l'affichage publicitaire. Les différents départements du Centre concernés (essentiellement la BPI et le CCI) font appel à des spécialistes dont le champ de compétence couvre l'événement national ou mondial qu'il est question de répercuter. La BPI a ainsi organisé des cycles de débats sur «L'Afrique noire » en 1979, «L'Amérique en crise » en 1980, «L'entrée de la Grèce dans le Marché commun » en 1981, la Révolution française en 1989. Le CCI a proposé plusieurs cycles sur l'environnement comme « L'environnement quotidien en Chine » (1982).

Quelques exemples de cycles

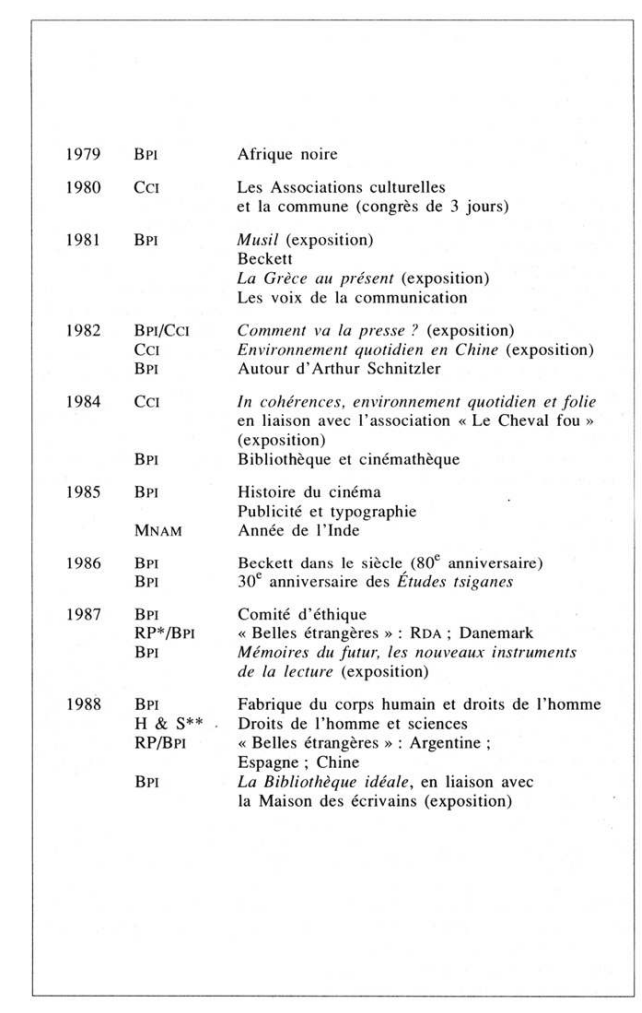

* RP : Revue parlée.

** H \& S cellule Histoire et société. 
tualité peut être d'ordre technologique et plusieurs cycles sur la communication ou les nouvelles technologies, depuis la conception assistée par ordinateur jusqu'à l'usage de la télématique, l'ont répercutée. L'actualité peut être aussi d'ordre littéraire comme en témoignent le cycle sur Musil, les cycles marquant des anniversaires : Beckett, Études tsiganes. Deux cycles enfin ont commémoré le bicentenaire de la Révolution : le « Forum de la Révolution » et «La famille, la loi, l'État: de la Révolution au code civil», ce dernier représentant la manifestation phare de l'activité de la cellule Histoire et société qui intervient sur la question du rapport entre faits de culture et faits de société. Avec "Le Front populaire », «Les droits de l'homme et la science ", "L'Europe au risque des cultures ", la cellule avait déjà marqué sa capacité à mobiliser des publics restreints sur des thèmes concernant l'histoire sociale et culturelle. La thématique comme la mise en scène de ces débats les situent dans un modèle qui tient à la fois du débat-colloque et du débat-spectacle. La conception adoptée est résolument celle de la concertation et positionne les débats hors du champ de la confrontation. temps qui permet un approfondissement. Les journées de rencontres entre spécialistes ne sont pas une simple répercussion de l'événement. Elles opposent à l'immédiateté du débat la production d'une réflexion. Avec les cycles de débats, on passe de la rencontre entre habitués au colloque réunissant des chercheurs. La tendance à l'évolution de l'espace public où on s'affiche pour acquérir une légitimité vers celui d'un espace réservé à la concertation (modèle de la société savante) est nettement marquée dans les deux cycles sur la Révolution. Le "Forum de la Révolution », organisé par la BPI, reste un lieu ouvert mais qui joue sur une autosélection des publics. «La famille, la loi, l'État ", organisé par la cellule Histoire et société est un cycle résolument fermé. Il se déroule en deux temps : un séminaire de neuf mois, réunissant des spécialistes; un colloque de deux jours ouvert à un public d'invités. Cette formule qui tend à se généraliser dans le Centre est très proche de celle qu'a lancée la cellule Espaceséminaire ( $c f$. p. 44 ), qui s'articule sur une double temporalité, alternant séances fermées où se confrontent des spécialistes et séances publiques, le soir. Cependant, en raison de sa liaison avec l'actualité du bicentenaire, le séminaire-colloque d'« Histoire et société » appartient à la catégorie des débats-colloques. le colloque universitaire: même type de préparation, même connivence avec les partenaires, non en raison de l'appartenance à un univers commun, mais de positions équivalentes dans le champ culturel. La différence vient du public qu'on perçoit comme fluctuant et à qui on s'adapte sans trop connaître ses demandes, en jouant au journaliste, en étant plus accessible. Les débats du «Forum de la Révolution » qui réunissaient des historiens participant au congrès mondial de la Sorbonne organisé au même moment en commémoration du bicentenaire ont été l'occasion d'une actualisation des pensées historiographiques. Elle fut imposée par les événements, dans le cas des rencontres Paris-Moscou (juin 1989), et des débats sur l'Europe centrale, la Chine et la Révolution française (juillet 1989). Mais elle fut aussi l'effet du lieu que les chercheurs vivent comme un élargissement de la scène universitaire. C'est ce qui marque la différence entre le "Forum de la Révolution» et le colloque de la cellule Histoire et société qui, lui, a gardé une forme très académique.

Beaucoup de manifestations sont accueillies par le Centre, qui n'est que prestataire de service (cas du colloque «La sexualité en Islam»). Dans le cas d'une collaboration, il 
devient organisateur de rencontres entre protagonistes qui feront émerger les idées forces, suggéreront le découpage thématique, la participation des intervenants. En répercutant un thème porteur, le Centre s'entoure des conseillers scientifiques qui sauront lui donner la valeur d'une manifestation culturelle.

L'ouverture de ce type de débat est généralement plus solennelle que dans les deux premiers cas étudiés. Plusieurs niveaux de médiations règlent l'entrée en scène des protagonistes. Leur forme et leur nombre varient avec l'importance de la rencontre. Certaines manifestations sont plus formelles que d'autres: les "premières » ou les cycles répercutant une actualité déjà chargée de prestige. La série de débats du « Forum de la Révolution" rassemble toutes ces caractéristiques. C'est une première technologique puisqu'il s'agit de radio-conférences, et d'une vidéo-conférence où les intervenants français sont reliés en direct avec des chercheurs étrangers travaillant sur le même sujet. La commémoration du bicentenaire tient une place importante dans le champ culturel. Le soir de la première radio-conférence, le directeur de la BPI ouvre le forum avant de passer la parole à l'animatrice qui introduira à son tour le thème du débat et les intervenants. Le soir de la vidéo-conférence, l'entrée en scène des invités sera médiatisée par plusieurs représentants de la hiérarchie : la présidente du Centre, le directeur de la BPI.

Les colloques organisés par une institution extérieure peuvent être également ouverts par des représentants de la hiérarchie du Centre qui introduisent les membres de l'organisme invité. On aboutit alors à une série de médiations qui précèdent l'entrée en scène du modérateur. Le type exemplaire en est le colloque sur "La sexualité en Islam » organisé par Les Cahiers de l'Orient qui déplaça également la présidente du Centre, le directeur de la BPI et où le début du colloque proprement dit fut précédé d'une présentation de l'institution invitée. Il s'agissait d'une première : porter un tel débat sur la scène culturelle est novateur. D'autre part, l'enjeu politique (on était en pleine "affaire Rushdie») imposait que soit rappelée, par les instances compétentes, la vocation de neutralité du Centre.

Après l'ouverture solennelle, l'animateur introduit la séance. Il commence par une présentation des participants dont il décline l'identité et la légitimité à intervenir. Elle s'appuie parfois sur l'énoncé de la biographie et la liste des productions de chacun. La légitimité est ainsi affichée (l'animateur peut montrer les livres des auteurs invités, dans un débat dont la finalité n'est pas de rendre compte de l'actualité éditoriale). Les tours de parole seront décidés par l'animateur en fonction de la place de chaque interlocuteur dans le champ, selon le principe de la territorialité. Reprenons l'exemple $\mathrm{du}$ «Forum de la Révolution», première séance. L'animatrice positionne chaque intervenant par rapport au thème central du débat: "Les livres ont-ils fait la Révolution?» Après l'avoir reformulé, elle distribue la parole selon le type d'énonciation suivant : «C'est une question que j'aimerais poser à J.-M. Goulemot » ou " Je vais demander à $R$. Chartier de répondre ». Chaque intervention fera référence à la précédente, reconnaissant le champ de compétence de son auteur, soit pour abonder dans son sens, soit pour en tracer les limites et aller plus loin. On cite aussi les ouvrages qui font référence. L'ordre du débat est dicté par les différentes façons de répondre à la question posée. C'est un ordre préparé. S'il y a divergence, il n'y a pas de véritable confrontation en direct, chacun connaissant les positions des autres. Selon les paroles d'un intervenant, «c'était sociable et poli ». 

débat d'écoles. Sous les diverses façons de répondre à la question posée "Les livres ontils fait la Révolution?» deux modèles d'historien s'affrontent. L'un se préoccupe des questions légitimes qui doivent être posées à l'histoire, comme celles qui déterminent ses ruptures, questions qui ne doivent pas attendre les moyens d'y répondre pour être posées (position défendue par Roger Chartier). L'autre cherche à accumuler les preuves (position de Robert Darnton, de New York), qui conteste le principe des hypothèses théoriques non vérifiées : «Oui, je suis d'accord avec la pluralité des lectures. Mais il faut la démontrer.»

Dans les radio-conférences, la technique était au service du débat, et la scène du forum pouvait être une transposition de la scène universitaire. Quand la technique envahit la scène - cas de la vidéo-conférence - le débat est détourné au profit du spectacle. Les organisateurs - côté Centre Georges Pompidou - ne se servent pas de la télévision comme les conférenciers de Los Angeles pour qui elle n'est qu'un instrument. Ils font de la télévision. Au cours de la vidéo-conférence, il n'y aura pas de débat d'écoles. Dans la grande salle du Centre, la technologie renforce les effets théâtraux, introduit des rituels spécifiques aux plateaux, des "suspens" liés aux imprévus de la transmission par satellite. L'animatrice - Christine Ockrent est une journaliste qui n'a pas de compétence spécifique pour conduire le débat. Pour le directeur de la BPI, le plateau français qu'il avait annoncé, au cours de l'ouverture de la série, comme « un grand plateau » est un plateau médiatique. Les intervenants Robert Badinter et Jean-Noël Jeanneney, y figurent respectivement comme président du Conseil constitutionnel et président de la Mission du bicentenaire au côté de Michel Vovelle, véritable conseiller scientifique du forum. Le plateau américain sera annoncé comme composé uniquement de scientifiques. Ces derniers resteront universitaires et soucieux de renvoyer l'image d'un campus passionné par la célébration du bicentenaire.

\section{Autour des expositions}

Certains cycles sont des compléments d'information autour d'un événement ou d'une exposition du Centre. Les manifestations internes à l'institution, même si elles font événement et mettent le Centre dans une position motrice, ne peuvent cependant pas être considérées comme des phénomènes d'actualité. Les rencontres qu'elles suscitent, en revanche, peuvent être identifiées aux précédentes. Elles s'apparentent au commentaire d'événements visuels destiné à un public. Il y a donc transmission d'une information, située dans un contexte qui est celui de l'activité la plus "visible» du Centre Georges Pompidou et consistant précisément à donner à voir des œuvres artistiques ou des objets de la culture contemporaine. Le commentaire n'est pas une explication/traduction des œuvres présentées au MNAM ou dans d'autres lieux d'exposition. Il apporte un complément d'information, c'est-à-dire qu'il parle autour de l'événement, mettant à contribution des sociologues de l'art, des philosophes de l'esthétique, mais aussi des spécialistes de la littérature, de l'édition, des historiens, des psychanalystes.

ieurs types de débats entrent dans cette catégorie. Leur différenciation repose sur une combinaison entre le style d'exposition concernée, le lien entre l'instance de représentation et l'instance productrice de débats. 
50 L'Espace-séminaire articule séminaires autonomes et séminaires liés à la programmation du Centre. Ces derniers, parmi lesquels «La philosophie des années 50 » et « Aspects de la pensée au Japon » viennent en écho des grandes expositions et ne sont pas en situation de métalangage.

51 Les débats de la "Revue parlée» sont conçus comme une exploration littéraire, poétique, philosophique du domaine couvert par l'exposition. Toutes les grandes expositions du MNAM ont fourni matière à débats dans le Centre mais c'est avec l'exposition sur Vienne qu'une série de rencontres est inscrite au programme dans l'ensemble de l'institution d'une façon qui occupe presque tout l'espace public. La « Revue parlée » organise quatre séries en résonance avec la manifestation :

Vienne, expérience freudienne ;

Autriche, itinéraire politique et social ;

Projets éducatifs et utopie de l'homme nouveau ;

Autriche, itinéraire culturel.

52 Autour du Japon des avant-gardes, la « Revue parlée » couvre aussi tout un ensemble de sujets. Pour Blaise Gautier, un débat est d'autant plus riche qu'il est « logé ». Il s'éloigne alors du rituel où les protagonistes s'affrontent sur des thèmes convenus et sans surprise pour le public qui connaît d'avance leur ligne de pensée. Il vient apporter des données sur le contexte historique, culturel, philosophique de l'exposition ainsi que sur ses résonances littéraires. Dans cette vocation d'approfondissement des expositions, la « Revue parlée » prend des allures de colloque.

Le café viennois inaugure aussi avec Vienne une nouvelle formule qui est celle du café littéraire, situé dans le forum, lieu central et de convergence des visiteurs. L'utopie du débat public est maintenue. Mais le profil qui se dessine tend vers la fusion de deux figures : celle du colloque, lieu de transmission où fonctionne un rituel hiérarchique et celle du salon littéraire, société de convives.

Le débat du CCI apparaît comme justification d'une exposition. Après Les années 50 : du béton au rock, un débat a été organisé par le service du Design, pour répondre à la controverse soulevée par les prêteurs des objets exposés. Pendant «Nouvelles tendances, mutation des styles » se tient un séminaire, animé par Umberto Eco. Entre l'exposition et le débat, il n'y a pas complémentarité mais diffraction. Il s'agit de transmettre un message sous deux angles d'attaque différents, l'un et l'autre étant voués à l'éphémère.

Une nouvelle tendance semble, cependant, se faire jour au ccI qui installerait le débatcolloque non plus en aval mais en amont d'une exposition. Il servirait à « fabriquer de la matière intellectuelle » pour mieux assurer un succès public qui viendrait après et serait en quelque sorte déplacé par rapport au colloque. Un séminaire entre spécialistes de niveau international vient d'être organisé autour de Virilio afin d'aboutir à une production écrite destinée à lancer une prochaine exposition. La manifestation orale laisserait des traces plus importantes que l'exposition tout en la promouvant. Le débatséminaire, à l'inverse du catalogue, joue un rôle moteur dans une nouvelle logique institutionnelle où l'exposition doit être lancée comme un événement publicitaire dont le succès sera mesuré de plus en plus à partir du nombre des entrées. 


\section{L'action de diffusion culturelle}

56 C'est celle qui s'apparente le plus à la transmission de savoir. Elle reste liée aux activités du Centre. Elle vise un public fidélisé. Les conférences destinées aux correspondants et aux adhérents du Centre en sont le modèle exemplaire. Elles ne constituent, cependant, qu'une partie de l'action de diffusion culturelle qui est conçue comme un processus plus global de sensibilisation à tous les secteurs d'activité temporaire ou permanente du Centre: art moderne, musique contemporaine, architecture, design, philosophie... L'activité « en salle » n'est qu'une approche parmi d'autres - dont la sensibilisation in situ - utilisée pour la formation des correspondants et des adhérents. Elle est aussi l'approche la moins spécifique et se différencie peu des autres conférences-débats organisées à propos d'une exposition si ce n'est par la référence au public.

Nous donnerons un exemple de débats organisés par la cellule liaison/adhésion autour de l'exposition Les Immatériaux. L'animatrice commence le débat par une lecture d'articles de presse, qui sont une façon d'introduire l'opinion publique. Puis elle énonce les questions adressées au service par le courrier des lecteurs. Le public est donc d'emblée intégré au débat et la thématique est donnée par le rapport qu'il entretient avec l'exposition. L'animatrice demandera ensuite aux intervenants, les maîtres d'œuvre de l'exposition, Jean-François Lyotard et Thierry Chaput, et le directeur du CcI, François Burkhardt, de répondre aux questions que pose la réception de l'exposition. On est dans la logique de la question-réponse où l'intervenant/concepteur de l'exposition est sollicité de développer le message dont on le suppose porteur et qui n'est pas totalement explicité dans sa production exposée.

Les conférences-débats organisées, pendant dix ans, par l'association Architecture et construction avec le ccI se rapprochent également du modèle de la transmission de savoir. Destinées à un public essentiellement composé d'étudiants en architecture et de professionnels, elles étaient conçues comme des cycles de formation, apportant une connaissance de haut niveau. Les objectifs en sont différemment formulés par les deux instances organisatrices. Le CcI, étant plus soucieux de maintenir l'image du Centre et d'affirmer sa capacité à attirer des personnalités de renommée internationale, lie la fonction pédagogique des conférences à la présence de grands architectes et au rôle d'enseignement magistral qu'ils peuvent tenir. Le bâtiment lui-même, symbole d'une architecture d'avant-garde, appelle ce type de formation. Pour les représentants de l'association, le succès des conférences - leur capacité à attirer les professionnels - fut lié à la mise en place d'une programmation en cycles, centrée sur des thèmes porteurs, et sur l'interdisciplinarité. Fonder la thématique des conférences sur le vedettariat conduit à la dispersion et à une politique peu adaptée aux capacités financières du Centre. Architecture et construction s'est donc orientée vers une politique de décloisonnement du discours des architectes et d'ouverture à d'autres langages. Elle a aussi essayé de fonder la légitimité des intervenants sur d'autres critères que ceux de la renommée.

59 Le fonctionnement de la conférence d'architecture repose sur le modèle type du débat « colloque ». L'animateur est issu du champ mais il est aussi journaliste et a donc une compétence de communication. L'entrée en scène des intervenants est précédée par une lecture de leur parcours et la présentation des thématiques. Selon la personnalité invitée, la salle est remplie d'une majorité d'étudiants ou attire nombre de journalistes. 
Le débat avec le public peut ainsi tourner à la conférence de presse quand le passage d'une vedette à Beaubourg est l'occasion de recueillir une interview.

\section{Les conférences}

60 Le genre qui tend à se développer de plus en plus, c'est la conférence ou la table ronde qui sont situées dans un huis clos, en dehors de l'actualité. La rencontre ne repose ni sur la théâtralisation d'un événement, ni sur la légitimation d'un auteur. Elle a lieu entre spécialistes qui à un moment donné échangent «en public» le fruit de leurs recherches. Trois types de production orale nous paraissent représenter le type idéal de la conférence :

- les conférences du MNAM sur des thèmes indépendants des expositions,

- le Cabinet de lecture, l'Espace-séminaire.

61 Dans les trois cas, il s'agit non d'approfondir des thèmes pour un public, mais d'aborder des sujets fondamentaux. Le choix est entièrement lié au programmateur, qu'il soit de la maison ou de l'extérieur, comme dans le cas du Cabinet de lecture qu'a lancé Étienne Balibar et qui a été repris par le Collège international de philosophie. Dans les trois exemples, la philosophie est prédominante, comme une sorte de fondement scientifique de la culture. Ces trois types de débats ne sont pas cependant identiques mais constituent comme les variantes d'un même genre qui se distinguent en fonction de leur place dans le Centre. Cette place correspond à des moments historiques différents qui définissent des conceptions différentes de l'espace public.

Le Cabinet de lecture s'inscrit dans le mouvement de l'« utopie Beaubourg » de porter le débat philosophique sur la place publique. Il reste une société de convives où la discussion se passe entre intellectuels appartenant au même réseau. Mais le cercle tient ses réunions "dans un hall de gare». Cependant, l'expérience dérivera vers le séminaire fermé, comme on le constate à l'écoute des enregistrements. Le Cabinet de lecture correspond à une forme de débat sans médiation, c'est-à-dire sans présentation des interlocuteurs et sans modérateur où le président de séance est aussi intervenant. D'emblée, il pose le problème du débat et annonce les différents exposés.

63 L'Espace-séminaire combine deux principes, le huis clos entre spécialistes et le débat public. Il fonctionne sur deux rythmes, celui du séminaire fermé, lieu de construction des concepts, et celui de la table ronde, où se succèdent les interventions destinées à rendre publiques les productions. Chaque manifestation donne lieu à une production écrite dans la collection l'Espace international des publications du Centre Georges Pompidou.

64 La conférence du musée élimine tout principe d'ouverture publique, qu'il s'agisse de parler devant ou pour le grand public. Sa finalité repose aussi sur la production de sens laissant une trace écrite. La conférence peut alors être considérée comme le relais qui rend possible la réalisation de la revue Les Cahiers du musée. La mise en scène en est évidemment très classique : le conférencier est introduit après une présentation de son parcours de recherche. Les tables rondes présentent des variantes. L'une d'entre elles, "Quoi faire aujourd'hui ? ou l'art est difficile » réunit plusieurs artistes autour du jeu de la présentation de soi. L'animateur, Bernard Blistène, annonce d'emblée son refus de «tomber dans le rôle du professeur» montrant les travaux des artistes à l'aide de 
projections et demande à chacun de parler de son art à travers une auto-définition. Ce qui sert d'habitude à introduire le débat en est pris pour thème.

Au cours d'une autre table ronde, «Le musée, but ou fin de l'art?» un membre du public, invité certes - il fait partie du réseau des organisateurs - mais pas au même titre que ceux de la tribune prend plusieurs fois la parole sans y avoir été convié. Il s'affiche, donc; mais comme il ne peut le faire comme les autres, dans un territoire reconnu, il conteste le fonctionnement du débat, substitue à la question posée le problème du droit d'expression en démocratie. C'est à partir de sa position hors statut - il n'est pas à la tribune et sans doute artiste méconnu qui n'a pas encore sa place dans un musée - qu'il parle. C'est aussi la situation spécifique du Centre Georges Pompidou, un musée pas comme les autres, qui l'y autorise. Les organisateurs sont pris au piège de ne pas pouvoir faire fonctionner un débat qui pose la question de la place du musée dans l'art à cause d'un auditeur qui s'impose comme contestataire de l'institution musée. L'intervenant intempestif donne à la table ronde, initialement préparée comme une série de conférences articulées autour d'une thèse centrale proposée par l'organisatrice, l'allure d'une conversation désordonnée, en remettant en cause les statuts.

Ces deux derniers exemples de conférences-débats organisées par le MNAM étaient évidemment des contretypes. Mais le jeu ou la dérive sont inscrits dans le huis clos quand il se passe avec des artistes pour qui le discours sur l'art est un discours emprunté au champ de la critique. Les caractéristiques attachées aux différents débats et qui en modèlent le style sont liées au lieu - ouvert ou fermé-, aux médiations précédant l'entrée en scène des intervenants, au fonctionnement de la prise de parole. Mais la position reconnue aux divers protagonistes dans le champ culturel et sur la scène de Beaubourg peut faire bouger les frontières entre les types idéaux.

Les quatre modèles présentés et qui ont été identifiés à la fois par leurs thématiques et par leur style ont bien entendu quelque chose de formel. Ils ne constituent que des formes construites, pôles ( $c f$. schéma p. 25) vers lesquels tendent les débats réels. Néanmoins ils permettent de mettre en évidence certaines évolutions. Ainsi, à reprendre l'esquisse de périodisation en fonction de cette typologie, il apparaît que l'évolution se produit du pôle (b) vers les pôles (c) et (d), l'axe b/d étant l'axe de la plus grande opposition. Cette évolution rend compte de l'orientation de plus en plus affirmée du Centre vers une structure para-universitaire. Si le débat mettant en scène la confrontation d'idées était exemplaire des débuts utopiques, on peut dire qu'aujourd'hui c'est la conférence qui est exemplaire d'un nouvel ordre intellectuel, reposant sur l'intervention du spécialiste légitimé, l'approfondissement de thématiques. Par son caractère solennel, la mise en présence de personnalités et d'un public, la conférence emprunte au style créé par la « Revue parlée ».

La conférence ou plutôt la série de conférences portées sur la scène pour un public souvent restreint par le système de l'invitation ou de l'auto-sélection apparaît de plus en plus comme l'aboutissement d'une production dont le Centre Pompidou est le siège. Par ses capacités d'accueil - que ne possède pas l'Université française-, son pouvoir mobilisateur, il est le lieu d'un nouveau genre : le séminaire de travail fermé au public, mais suivi d'une exposition quasi solennelle et souvent spectaculaire dont la conférence n'est qu'une des formes, au côté de la production écrite ou de l'exposition visuelle. 


\section{NOTES}

1. Jean-Claude Bonnet, "Les morts illustres", Les Lieux de mémoire. La nation, vol. 3, sous la direction de Pierre Nora, Gallimard, 1986.

2. Jean-Claude Bonnet, « Les morts illustres ", Les Lieux de mémoire..., op. cit.

3. J.-L. Austin, How to Do Things with Words, Oxford, 1962.

4. Isaac Joseph, «Éléments pour l'analyse de l'expérience de la vie publique », Espace et société, $\mathrm{n}^{\mathrm{o}} 38-39,1981$, p. 57-76.

5. Ibid. 


\section{Chapitre 3. Les acteurs des débats}

1 Une fois située la chronologie des débats ainsi que leur typologie, nous pouvons nous intéresser davantage aux divers protagonistes qu'ils impliquent. A l'évidence, ceux-ci se répartissent en quatre groupes distincts :

- les organisateurs, concepteurs ou programmateurs des débats, au premier chef, les différentes instances du Centre,

- les animateurs,

- les participants proprement dits,

- le public.

Cette répartition est fonctionnelle, dans la mesure où la même personne peut se trouver à la fois faire partie du premier et du second groupes, ou passer du troisième au quatrième selon les débats.

\section{Les organisateurs}

Il faut ici distinguer trois cas de figure. Premier cas, celui où une instance du Centre est prestataire de service à l'égard d'un groupe ou d'un individu doté d'un projet (par exemple le débat sur la sexualité en pays musulman; ou encore le débat sur la laïcité organisé par l'association Alethe). Bien évidemment, l'organisateur n'a pas une autonomie totale. Il lui a fallu discuter de son projet avec un ou plusieurs responsables du Centre, et celui-ci a fini par être retenu en fonction de certains critères. La Salle d'actualité de la BPI est ainsi constamment sollicitée d'organiser des débats sur les thèmes les plus divers, dont la plupart ne sont pas retenus. Il n'en reste pas moins que si l'organisateur a su faire accepter son projet, le Centre n'intervient plus guère, sinon sous la forme d'une assistance matérielle (invitations, etc.) dans l'organisation du débat.

Deuxième cas : celui d'une collaboration entre une instance du Centre et un concepteur extérieur. La plupart du temps, il s'agit alors d'une demande du Centre adressée à un individu ou à un groupe, ou d'une collaboration régulière engagée à partir d'un premier contact entrepris plutôt dans la première hypothèse. Quoi qu'il en soit, l'organisation $\mathrm{du}$ débat est alors davantage négociée. Le Centre est pleinement partie prenante de l'organisation, fait valoir des contraintes propres. Celles-ci peuvent être de 
programmation, matérielle (nombre de débats par semaine, liberté relative des salles) ou thématique (il y a déjà eu un débat sur ce thème, etc.). Elles peuvent porter aussi sur la forme du débat: la demande porte sur un type particulier d'intervenants, ou une forme originale (exemple : le Cabinet de lecture). L'interlocuteur choisit des thèmes, des personnalités devant intervenir, un animateur en fonction de ces critères, puis le projet est rediscuté. Enfin, la phase terminale consiste à arrêter une liste définitive de participants, une date et à inscrire le débat dans la programmation du Centre. Souvent, après le débat, celui-ci fait l'objet d'une évaluation, certes plus ou moins sommaire. Ce cas de figure parait offrir un net avantage sur le précédent: mettant le Centre en position de commanditaire, et son interlocuteur en situation de répondre à cette commande, il favorise éminemment la concertation et conduit nécessairement à davantage préparer le débat. Celui-ci a sans doute plus de chances d'être pensé et élaboré dans ce cas que dans d'autres, ce qui n'implique a priori rien sur la réussite éventuelle du débat, celle-ci dépendant de toute une série d'autres facteurs.

Troisième cas : le Centre est directement organisateur du débat, par le biais d'une de ses instances ou même directement de la présidence ( $c f$. le débat télévisé organisé par Hélène Ahrweiler, à propos de la censure, à l'occasion de la condamnation par l'Iran de Salman Rusdhie). A part ce dernier exemple, atypique, c'est le cas le plus fréquent. On a vu comment progressivement chacune des instances du Centre avait spécialisé une personne, ou une cellule, dans l'animation de débats. Chacune d'entre elles développe sa stratégie propre, et commandite des débats qui s'organisent le plus souvent dans un projet d'ensemble. Les organisateurs sont ici confondus avec les commanditaires et les maîtres d'œuvre du débat. En revanche, ils n'assument que rarement la tâche de l'animation et la confient à un intervenant extérieur la plupart du temps.

\section{Les animateurs}

$6 \quad$ Les animateurs des débats se laissent aisément répartir en deux grandes catégories : les animateurs «professionnels » et les animateurs «occasionnels ». Mais là encore cette distinction est prise ici en un sens fonctionnel. Tel animateur professionnel pourra fort bien être plutôt un universitaire, tel occasionnel, un journaliste, même si c'est ordinairement plutôt l'inverse.

Nous dirons que nous avons affaire à un animateur professionnel s'il remplit trois séries de critères : animer plusieurs débats,

animer des débats sur des thèmes divers,

10 animer des débats dans d'autres lieux que le Centre Pompidou.

11 De fait, la plupart des animateurs professionnels sont journalistes, ou l'ont été à un moment de leur carrière. C'est par exemple le cas de Roland Jaccard, journaliste au Monde, fréquemment sollicité pour animer des débats consacrés à la psychiatriepsychanalyse, ou plus généralement aux sciences humaines, tant à la BPI qu'à la « Revue parlée ». Mais on trouve aussi dans cette catégorie des animateurs qui ont pris en charge un cycle, ou qui sont ponctuellement sollicités, et qui sont universitaires (Françoise Gaillard ou Christine Buci-Glucksmann pour le Cabinet de lecture de la BPI). Il faut aussi considérer le cas de ceux qui, animateurs, sont aussi les organisateurs du débat, et qui font partie des instances du Centre: Blaise Gautier pour la «Revue 
parlée », Philippe Arbaizar à la Salle d'actualité de la Bpi dans un premier temps. S'il fallait toutefois choisir un nom pour représenter cette catégorie d'animateurs, ce serait sans doute celui d'Antoine Spire qui s'imposerait, tant le nombre et la diversité des débats qu'il anime sont impressionnants. En outre il anime aussi bon nombre de débats à la Fnac, et bien entendu à France-Culture.

Il semble que son itinéraire ne le conduise à croiser le Centre que vers 1984. Alors journaliste au Matin de Paris, son intervention dans les débats se développera considérablement avec le cycle de débats Bpi/Matin de Paris en 1985-1986 (l'information, la France pluriculturelle, la pensée 68, le Front populaire, etc.). Puis il deviendra tout naturellement un des animateurs réguliers de la Salle d'actualité, jusqu'à finir par en être quasiment le coprogrammateur, et proposer l'animation de débats à certains de ses collaborateurs d'autres lieux (Michel Field, qui vient du Panorama de France-Culture). Il se définit lui-même comme un professionnel de l'organisation de débats, cherchant à promouvoir un quasi-statut pour ce type d'activités: "Il faut faire appréhender la notion d'animation comme spécifique. L'animation suppose un suivi d'ensemble et de conception du rôle du débat. A Pompidou, on sous-estime l'élaboration intellectuelle que suppose la préparation du débat, et en conséquence le salaire de l'animateur » (Antoine Spire, entretien avec les auteurs).

Davantage liés à un sujet ou à une question, les animateurs occasionnels peuvent souvent être l'un des participants au débat. Ce sont des spécialistes, dont le rôle peut varier d'un modèle qui se rapproche davantage de la présidence de colloque, à caractère plus ou moins honorifique (par exemple Claude Bourdet, « La fin des terres promises ", 13 mars 1980 à la BPI, ou encore André Chastel, «Pour Henri Focillon », 23 juin 1986, «Revue parlée »), à l'animation proprement dite. D'une manière générale, l'animateur occasionnel est le plus souvent un universitaire ou un spécialiste auquel on a fait appel pour animer un débat déterminé. Mais certains journalistes peuvent entrer dans ce cas de figure (Pierre Bouteiller, «L'art contemporain dans la presse écrite », 27 novembre 1986, MNAM). Jean Lebrun, de France-Culture, est aussi animateur occasionnel d'un des débats du Forum de la Révolution ("La Vendée et sa mémoire », 31 mai 1989): " Ça correspond au genre d'émissions que je fais ; le thème était d'actualité. "

14 Le style d'animation est évidemment différent d'un animateur à l'autre. L'un, plus extérieur, cherchera avant tout la réussite du débat comme tel, dans le temps et le lieu qui sont les siens. Inversement, il pourra être tenté « d'en rajouter » pour légitimer sa place d'animateur et souligner sa propre connaissance des œuvres ou des questions abordées. Un autre aura tendance à se confondre avec les participants, et ne pas distinguer la place de l'animateur. Mais il sera souvent aussi plus attentif aux nuances, et soucieux de replacer le débat dans le long cours d'un travail ou d'une recherche. Tel débat ne constitue qu'un moment d'une activité plus large, qui se poursuivra dans des livres, articles, colloques, ou d'autres débats. Ainsi Lise Andries, chercheur au CNRS, commente le débat qui inaugurait le Forum de la Révolution, et qu'elle anima : "Je connaissais peut-être trop bien tous ces gens pour que ça apparaisse spontané. Je devais être participante au départ. Tous les intervenants présents ont l'habitude de se retrouver pour parler de ce sujet deux ou trois fois par an dans des congrès. C'était une répétition universitaire. Mais il y avait le public... »

Peut-on néanmoins, d'un débat à l'autre, tenter une typologie générale des animations, en fonction de la manière d'animer? Il semble que oui, tant le style dépend ici de la 
personnalité de l'animateur plus que de la thématique du débat ou même de la catégorie socio-professionnelle d'origine de l'animateur. Plusieurs «figures" d'animateurs se laissent ainsi définir.

\section{L'animateur fonctionnel}

16 Celui-ci joue son rôle a minima. Il présente sobrement les participants, le thème de la discussion. Il se refuse à intervenir, ou ne le fait que contraint et forcé, s'il sent que le débat languit, dévie, ou échappe. C'est bien entendu le plus souvent un animateur occasionnel. Mais ce peut être aussi un professionnel que la qualité des invités impressionne ou tout simplement qui fait de cette neutralité, voire de cette froideur, la marque de son professionnalisme (par exemple Christine Ockrent lors du débat «Révolution française » avec Jean-Noël Jeanneney, Jean Tulard, Michel Vovelle, Robert Badinter et Madeleine Rebérioux pour la France, et Keith S. Baker et Line Hunt en duplex des États-Unis). En général, ce style d'animation convient plutôt à un débat fortement centré, aux enjeux bien établis, avec des participants plutôt universitaires. Il se rapproche de la présidence de colloque, et donc suppose un cadre extérieur au débat, plus ou moins implicite, qui le sous-tend et le justifie.

\section{L'animateur vedette}

A l'opposé de l'animateur fonctionnel, l'animateur vedette est au centre du débat. Si ce n'est pas lui qui l'a organisé, il en est du moins le maitre d'œuvre, de bout en bout. Ce débat est ainsi le sien, pour le meilleur et pour le pire. Ultimement, c'est pour lui que le public est venu et accessoirement pour le thème : le modèle est ici clairement celui de l'animation télévisuelle, avec ses rendez-vous fixes. On pense à Pivot ou à Polac. Au Centre, il n'y a pas de telle personnalité disposant à elle seule d'un créneau. Mais certains débats tendent à se rapprocher de ce modèle : ceux animés par Antoine Spire, qui, on l'a vu, revendique son rôle de professionnel du débat. Nécessairement, son style d'animation, fait d'un mélange de rondeur bienveillante et de provocation, s'impose à travers tous les débats qu'il conduit. Son but est que l'espace du débat existe comme tel. Il mise donc sur les oppositions, réelles ou supposées, entre les participants, le caractère paradoxal, voire choquant de certaines thèses, sollicite les silences, cherche à faire du débat un événement. En revanche, il se méfie beaucoup des perturbateurs venus de la salle dont il connaît plusieurs échantillons. Il est habile à ne pas leur donner la parole, ou à différer leur prise de parole, qui vient parasiter le débat. Il s'engage tout entier dans l'animation, interrompt un intervenant trop fastidieux, sollicite un intervenant timide. Il est évident que ce type d'animation convient le mieux aux débats d'actualité ou aux débats à forte charge conflictuelle.

Un autre cas de figure voisin engendre cependant un style d'animation tout différent : il s'agit de Blaise Gautier, dont la «Revue parlée » constitue une sorte de rendez-vous régulier. Son style d'animation, quand il anime, est latéral : il présente des invités, puis s'efface. Il peut même confier l'animation effective du débat à quelqu'un d'autre, mais garde néanmoins le contrôle de la dynamique du débat. Là, le débat est moins un événement en tant que tel qu'un maillon dans la longue chaîne de la "Revue parlée " qui constitue le cadre fixe. De fait, elle se prête à des activités très diverses (lectures, spectacles) qui ne sont pas toutes, loin s'en faut, des débats. Là, ce n'est pas le jeu de 
l'animateur qui prime, mais le cadre du débat, et le rôle de Blaise Gautier tient à ce qu'il en est le concepteur.

\section{Le commentateur}

19 Moins présent que l'animateur vedette dans la dynamique du débat, le commentateur ne s'efface pas pour autant. Il reprend la parole après chaque intervenant, et place un fragment de commentaire; celui-ci peut être de blâme (rarement) ou de louange (souvent), mais la plupart du temps il porte sur le fond: rapprochement entre ce qui vient d'être dit et une autre théorie, ou les travaux d'un autre intervenant; reprise parfois pédagogique quand il a cru que le public n'avait pas saisi le discours; cheville entre une intervention et une autre. Le jeu du commentateur est de donner au débat une continuité. Il est nécessairement plus consensualiste que l'animateur vedette même s'il ne le cherche pas : ses reprises ont en effet tendance à unifier le débat, à gommer les oppositions de personnes au profit de l'éclairage de la question posée. Le commentateur a un style particulièrement pédagogique: il se rapproche de l'enseignant qui a invité un collègue dans son séminaire. Mais il ne l'est pas nécessairement : un journaliste soucieux de rendre compréhensible un débat difficile adoptera volontiers cette attitude. De fait, elle accompagne souvent les débats « difficiles" pour un large public (par exemple l'Espace séminaire, ou encore la Salle d'actualité).

\section{L'animateur désinvolte}

Détaché apparemment du débat il ne l'est pas à la manière de l'animateur fonctionnel. Il s'amuse de ce débat, du sérieux des intervenants, de leurs affrontements. Il intervient peu, mais garde tout le temps une attitude de distance critique qui n'échappe à personne. Il cherche pour partie à relativiser le débat, pour partie au contraire à le dramatiser, en comptant sur l'effet de son attitude sur les participants, ou le public. A la limite, on ne sait pas s'il s'ennuie vraiment d'être là, ou au contraire s'il joue à la perfection son rôle. Sa marge de manœuvre est étroite : à trop forcer dans ce sens, il risque de décourager certains participants, ou au contraire d'être pris à partie. Cette ambivalence fait que son style d'animation convient à des débats très opposés : soit des débats aux enjeux faiblement marqués, qui rassemblent de manière occasionnelle plusieurs intervenants sur un thème un peu arbitraire. Il faut alors que le débat s'engage, malgré tout. Soit au contraire le débat passionné : sa manière de sembler dire «ce n'est qu'un débat » permet de pacifier l'affrontement, qui menace le débat luimême.

21 Ces quatre types d'animation n'épuisent pas bien entendu tous les styles d'animation: d'une part, parce que on ne rencontre jamais de type «pur »; d'autre part, parce que le même animateur peut jouer sur plusieurs registres, et passer d'un style d'animation à l'autre selon la dynamique qui s'engage. Mais cette classification esquisse une typologie qui recouvre la typologie des débats envisagée plus haut. Elle retentit enfin sur les attitudes que vont devoir prendre les participants. 


\section{Les participants} Un dépouillement des archives sur sept ans (1978-1984) laisse apparaitre, parmi les participants les plus fréquents, outre les animateurs ou concepteurs de débats (Jacques Cellard, ou, pour les seconds Philippe Arbaizar à la BPI, Bernard Blistène et Jean Clair au MNAM) des participants réguliers. Il faut toutefois prendre ces chiffres comme des indicateurs plutôt que comme la mesure effective du nombre de participants, pour deux raisons : les données des archives ne donnent pas à coup sûr tous les participants (notamment pour la "Revue parlée ») et les données fournies, qui correspondent à la programmation, peuvent bien ne pas convenir aux performances effectives. En revanche, il semble qu'on puisse s'en servir pour établir des comparaisons. De ce point de vue, on voit nettement apparaître une catégorie de champions des débats (plus de six occurrences) : Louis Marin, Edgar Morin, Daniel Sibony, Philippe Sollers, ces deux derniers rivalisant dans l'obtention de la palme. Une autre, composée des participants réguliers (de trois à cinq occurrences), parmi lesquels Jean-Marie Benoist, Jacques Bouveresse, Michel Butor, Michel Deguy, Dominique Desanti, Jean-Toussaint Desanti, Jean-Marie Domenach, Serge Fauchereau, Roland Jaccard, Albert Jacquard, Yves Lacoste, Jean Lacouture, Marc Le Bot, Yves Michaud, Serge Moscovici, Jérôme Peignot, Michelle Perrot, Maurice Olender, Jean-Michel Palmier, Marcelin Pleynet, Christian Prigent, Denis Roche, Guy Scarpetta, Paul Virilio. Soit quinze universitaires, six journalistes et trois " purs écrivains ». Ou encore, cinq philosophes, sept critiques d'art, trois sociologues, un géographe, une historienne, pour ceux auxquels on peut assigner une appartenance disciplinaire majeure. On peut considérer cette proportion comme grossièrement représentative de la composition des débats : l'étude de la vingtaine de participants qui totalisent deux débats le confirme. On confirme ainsi les grandes priorités thématiques. A noter que si certains intervenants sont fidèles à l'une des instances du Centre (Dominique Desanti à la BPI, mais Jean-Toussaint Desanti à la «Revue parlée »), d'autres interviennent dans toutes les instances, comme Jérôme Peignot ou Serge Fauchereau, sans compter nos quatre champions, à la notable exception de Daniel Sibony, dont la participation à la « Revue parlée » (avant la page "Rebonds" de Libération ?) évoque la compulsion de répétition. On voit ainsi s'esquisser des réseaux d'affinités et de complicités, mais aussi des logiques d'intervention : là encore, les critiques sont les plus polyvalents.

L'étude quantitative rencontre ici ses limites: si le nombre de débats auxquels il participe est un indice de la notoriété et de la légitimité d'un intervenant, il ne suffit pas à préciser son statut. Une typologie des participants s'impose, en fonction de leurs modalités de participation. 


\section{Le maître respecté} locale (il est souvent plus jeune). C'est son savoir qui le qualifie, plus que sa position. Son attitude est souvent moins bienveillante, notamment à l'égard des généralistes qui s'aventurent sur son champ, surtout si ce sont des vedettes (ainsi, tout philosophe universitaire se fera un devoir de marquer la distance, et, s'il en a le bonheur, de relever une erreur dans le discours d'un «nouveau philosophe » ou d'un " généraliste " comme Sollers). En revanche, sa courtoisie est totale à l'égard des autres spécialistes ou des élèves. Il est aussi le seul à se permettre parfois quelque impertinence à l'égard des maîtres. S'il est étranger, son prestige en est rehaussé, mais son ignorance, réelle ou feinte, des enjeux hexagonaux le disqualifie dans la dimension agonistique du débat (par exemple les Américains comme Keith Baker dans les débats de la BPI consacrés à la Révolution). Le spécialiste reconnu peut parfois être très ennuyeux : c'est son droit, et sans doute sa revanche sur les vedettes. C'est aussi la hantise des animateurs et des organisateurs. Il peut en revanche et doit se montrer extrêmement pédagogue et même brillant si le débat est fortement conflictuel (type Jean-Clément Martin face à Reynald Secher sur la Vendée).

\section{Le spécialiste contesté}

Spécialiste, car il est souvent universitaire, et a écrit un ou plusieurs ouvrages sur le sujet, ses thèses sont fortement controversées. Cette controverse peut être locale et technique, ou au contraire générale. Souvent le débat est organisé de manière à faire apparaître cette contestation, quand ce n'est pas pour « coincer » le spécialiste contesté (cela dépend de sa légitimité générale et de la nature du thème : par exemple Reynald Secher à propos de la Vendée, ou encore Jean-Pierre Changeux sur l'homme neuronal face à Dominique Lecourt, 12 octobre 1983, BPI, Cabinet de lecture). Le spécialiste contesté a plusieurs stratégies à sa disposition : chercher des alliances, ou au contraire 
prendre la figure du perturbateur. Il peut aussi choisir le rôle de la victime tombée dans un traquenard. Selon la stratégie choisie l'animateur verra sa tâche plus ou moins facilitée.

\section{La vedette}

Coqueluche des débats, la vedette a une forte légitimité médiatique. Elle parle bien, avec aisance et cette pointe d'assurance qui trahit son mépris pour le spécialiste myope. La vedette est en effet généraliste, souvent philosophe ou affectant de l'être. Son intervention s'apparente toujours à une performance. L'absence d'une vedette annoncée est une catastrophe pour l'organisateur et pour l'animateur, moins pour les autres participants (par exemple Bourdieu lors d'une des séances publiques de l'Espace séminaire consacrée aux "années cinquante »; il avait participé aux séances fermées, mais un brutal empêchement devait l'empêcher d'être là le soir), s'ils sont spécialistes ou impétrants. La vedette est souvent complice de l'animateur, mais peut aussi choisir de le ridiculiser. Le débat tourne au spectacle si plusieurs vedettes se disputent le premier rôle. La souveraineté de la vedette se marque à son indifférence à la manière dont évolue le débat, même si c'est à son désavantage. La vedette peut aussi refuser de jouer ce rôle et s'en tenir à celui de simple participant. Les vedettes sont bien évidemment les champions de la participation quantitative aux débats (P. Sollers, D. Sibony). Mais on peut aussi avoir le cas de figure d'une vedette d'un soir (Jean Maurel, Espace séminaire 1989).

\section{Le généraliste discret}

31 Inconnu, ou plus exactement connu des seuls spécialistes et animateurs de débats et d'une fraction informée du public, il est le plus souvent, à la différence de la vedette un généraliste sectoriel (M. Arkoun sur l'islam, R. Chartier sur l'histoire des mentalités, $O$. Corpet sur les revues, etc.). Il doit sa position soit à sa capacité de maîtrise du champ et à son autorité propre (on peut dire qu'il s'agit là d'une vedette en voie de constitution), soit à sa position institutionnelle, soit encore à sa qualité propre de « débatteur » (E. Balibar), quand ce n'est pas aux trois réunis (R. Chartier). Le généraliste discret (il vaudrait mieux dire peu connu) constitue souvent l'élément quasi indispensable d'un débat. Il est d'ailleurs à mi-chemin de l'Université, de la presse et de l'édition. Il a le choix entre plusieurs attitudes : soit il lorgne vers la vedette, et mise sur la virtuosité ; soit il s'identifie avec quelque impatience au maitre et adopte un discours d'autorité parfois mal assuré ; soit enfin il assume pleinement sa posture de médiateur culturel averti, et se fait à la fois le pédagogue du débat et le découvreur de talents. Il est souvent en posture d'animateur-bis, quand il ne l'est pas directement (F. Gaillard, C. Buci-Glucksmann). Il est l'adversaire idéal du spécialiste contesté, mais peut aussi se montrer un contradicteur redoutable pour la vedette. Il est menacé soit par la position de rivalité avec la vedette, soit par celle de rivalité avec le maître. Son discours devient alors artificiellement brillant, ou compassé.

\section{L'inspiré jaculatoire}

Personnage qu'on rencontre davantage dans les débats littéraires que dans les débats d'idées, l'inspiré jaculatoire est porteur d'un message ou du moins d'une parole. Il ne 
débat pas, il profère, sur un ton souvent poétique ou méditatif. Le débat lui est prétexte à suivre à haute voix sa méditation propre. En fait, il a plutôt tendance à perturber le déroulement du débat, sauf si celui-ci lui est consacré («Revue parlée »). Dans ce cas, sa virtuosité apparente sa parole à celle de la vedette. Seule sa notoriété est moins grande. Il se peut qu'il aspire au statut de vedette mais ce n'est pas nécessaire.

\section{L'impétrant}

Jeune la plupart du temps, l'impétrant se caractérise par son manque de légitimité. Totalement inconnu du public, il participe au débat soit en raison d'une compétence particulière, mais encore cachée, soit par le hasard d'une rencontre avec l'organisateur, qui ne déteste pas donner ainsi une chance à un jeune et apparaître le cas échéant comme un découvreur de talents, soit encore pour venir combler un vide à la suite de défections. Souvent il ne connaît que de nom les autres participants, qui ne le connaissent pas du tout, et le lui font sentir (par exemple en oubliant ou en écorchant son nom : ce statut l'apparente à celui de l'étranger), sauf si ce sont des maîtres, ou des généralistes bienveillants. Il ne dispose que de l'espace du débat pour s'affirmer : aussi y met-il toute sa conviction, mais il laisse également voir son manque de savoir-faire. Sa tactique oscille entre deux extrêmes : se replier sur son savoir et ce qui le légitime dans le débat; sa modestie tranche alors sur l'attitude des autres participants et n'a d'égale que celle du maître. Mais dans son cas elle se confond aussi avec le manque d'assurance. Ou bien au contraire il joue un gros coup : tenir des propos ouvertement paradoxaux, et surtout attaquer la vedette ou se montrer impertinent à l'égard du maître. C'est risqué, mais payant.

Cette typologie ne doit pas conduire à figer les intervenants dans des rôles: elle constitue un instantané, et tel ou tel peut bien parcourir différentes étapes: c'est évidemment le cas pour le dernier type (« l'impétrant»), mais aussi pour le spécialiste, ou pour le généraliste. Ces stratégies sont constitutives d'itinéraires qui renvoient à la globalité du champ, et au partage de la légitimité qui s'y effectue.

\section{Le public}

L'étude du public a été a priori exclue du champ de l'enquête. Néanmoins il est difficile de l'ignorer, surtout quand on prend en compte les acteurs du débat. Celui-ci en est un en effet, tant par son nombre que par son comportement. En outre, du public viennent souvent des interventions qui font partie intégrante du débat. Nous allons successivement faire quelques remarques sur les interventions, puis sur le nombre et le comportement.

\section{Les interventions du public « de la salle »}

Le débat touche à sa fin. Déjà certains, qui avaient des fourmis dans les jambes, font mine de se lever. C'est le moment que choisit l'animateur pour poser la question : «Il faut maintenant donner la parole à la salle. Y a-t-il quelqu'un qui souhaite intervenir? »Ce moment de l'animation est délicat. En même temps, il constitue une arme puissante aux mains de l'animateur, qui peut ainsi interrompre, ou du moins presser un orateur qui s'éternise, ou au contraire, en réduisant ce moment à la portion 
congrue, considérer que pour l'essentiel le débat s'est déroulé à la tribune. Quoi qu'il en soit, ce moment fait partie de la rhétorique obligée du débat comme débat démocratique. La démocratie ici visée y est cependant souvent l'objet de malentendus. Pour l'animateur et les participants du débat, c'est l'occasion de revenir sur un aspect trop rapidement évoqué, ou de préciser une intervention un peu obscure. Bref, ils conçoivent les interventions de la salle comme des questions adressées à la tribune. Souvent au contraire, l'intervenant de la salle est davantage soucieux de sa propre parole, et conçoit bien son intervention comme une intervention dans le débat, porteuse d'une information ou d'une opinion non encore représentée. Pour lui, qui prend l'utopie du Centre au sérieux, la démocratie a été mise à mal dans la première partie du débat, et il s'agit de la rétablir. Faire paraître la diversité des opinions, leur irréductibilité aussi, lui semble souvent plus important que de contribuer positivement à une recherche commune. Ces interventions se distinguent donc en fonction de l'accent plus ou moins fort mis sur ces aspects, de leur plus ou moins grande intégration au débat: il s'agit souvent d'une "parole intempestive », du moins aux yeux des organisateurs et animateurs.

37 On ne peut toutefois ignorer que certaines stratégies conduisent les organisateurs à «faire la salle » : en disposant de quasi-intervenants dans la salle, ils constituent ainsi un public privilégié qui permet au débat de se poursuivre sur la même lancée, ( $c f$. plus loin : les « pairs » et les « copains »).

\section{Les pirates}

38 Souvent agressifs, parfois pathétiques, les pirates s'emparent de la parole dès qu'elle est offerte, parfois même sans attendre qu'on l'offre. Leurs interventions n'ont le plus souvent absolument aucun rapport avec le débat. Souvent repérés d'emblée par l'animateur, s'il est exercé, ils lui posent le dilemme suivant : comment perturberont-ils le moins le débat? Est-ce en leur donnant la parole ou en la leur refusant ? Les pirates sont là pour mémoire.

\section{Les assoiffés de savoir}

Paradoxalement, leurs interventions sont souvent marquées d'une irritation profonde contre la forme débat, et peuvent parfois être plus terroristes encore que celles des pirates. C'est qu'ils sont venus pour apprendre, et installent avec rigueur un dispositif dans lequel la tribune a le vrai, et doit éclairer la salle. Tout désaccord irréductible, toute vision trop nuancée, tout souci trop problématique les laisse sur leur faim et leur paraît souvent le fait d'un stratagème pour ne pas dire ce que les participants doivent nécessairement savoir. Pour eux, le débat ne saurait être réussi s'il ne parvient pas à des conclusions d'apparence définitive sur la question traitée. Fréquemment autodidactes, leurs interventions témoignent d'un positivisme spontané. «Quelle est la vérité ?», voilà leur question favorite.

\section{Les bons élèves}

Ce sont évidemment les enfants chéris de la tribune, animateur comme participants. Ils posent toujours de bonnes questions, dans les bons termes, au bon moment. Leurs questions sont des vraies questions qui donnent aux participants l'occasion de constater qu'ils ont été compris, et en plus de reprendre la parole pour préciser un 
point. En général assez au fait de la question traitée, les bons élèves sont souvent soit étudiants, soit enseignants. Il est rare qu'ils soient déçus par un débat. Ils ont les deux catégories précédentes en horreur.

\section{Les disciples}

41 Les disciples doivent être soigneusement distingués des bons élèves. Ils sont attachés à une personne qui participe au débat, non au thème ou à la question posée. De cours en conférences ou en débats, ils suivent toutes les manifestations publiques de leur héros. Leurs interventions ont pour but, parfois maladroitement, de le mettre en valeur, et souvent de le défendre dans un débat où sa parole n'était pas hégémonique. Ils s'efforcent donc de lui permettre de reprendre la parole, espérant parfois s'attirer une remarque amicale du maitre. Les plus convaincus des disciples eux, s'en prennent avec énergie aux contradicteurs de leur idole. Ils peuvent être redoutables pour l'animateur si, à la suite d'une difficulté de programmation, le maître attendu n'est pas là : rien ne les fera renoncer à l'idée qu'il s'agit d'une manœuvre destinée à l'empêcher de parler. Une autre variante de disciple peut s'avérer redoutable : ce sont les convertis, qui ont depuis peu renoncé à un maître décevant pour se tourner vers un autre.

\section{Les copains}

Les copains constituent le plus souvent une petite fraction du public, bienveillante et silencieuse. Assis dans les premiers rangs, leur présence familière rassure l'orateur, surtout s'il n'est pas chevronné. Ils savent qu'ils auront à le rassurer, après le débat, sur la qualité de sa prestation. Les copains interviennent dans trois cas: si le débat ne démarre pas, ou menace de sombrer, à cause des interventions des pirates ou des disciples; ils posent alors une question de complaisance, destinée à le remettre sur les rails, ou à encourager une salle timide. Parfois, surtout s'ils sont copains avec l'animateur, le principe d'une telle intervention a été convenu avant le débat. Le deuxième cas est celui où leur copain est victime d'une agression caractérisée, de la part d'un ou de plusieurs des membres de la tribune; ils se rangent alors publiquement de son côté. Le dernier cas est celui où le débat, peu nombreux, se réduit quasiment aux copains. Il prend alors un tour de familiarité et de conversation amicale un peu plus formalisée qu'à l'ordinaire. Ils affichent leur familiarité avec l'orateur en le tutoyant, et vont boire un pot avec lui après le débat. Les copains méprisent les disciples et les bons élèves. Nombre d'entre eux sont des pairs.

\section{Les pairs}

Les pairs sont avec les copains les seuls à tutoyer les orateurs, quoique pas systématiquement. Leurs interventions sont un prolongement du débat qui a eu lieu à la tribune. Ils prennent le même ton que les orateurs, parlent parfois aussi longuement qu'eux. La présence des pairs dans un débat est un signe de l'importance du débat pour le champ considéré. En général, les pairs ne se déplacent pas s'ils n'ont pas été officiellement invités à faire partie de la tribune. Leur présence a donc toujours une signification forte. Dans certains cas, il s'agit de contester la programmation. Pendant tout le débat, la présence du pair aura été comme une statue du reproche. Son intervention mettra en évidence les erreurs dans la conception du débat, où il aurait dû avoir une place centrale. Dans d'autres cas, la nature même du débat l'annonçait 
comme un événement. D'emblée donc, le débat s'engage entre pairs. Les autres intervenants sont rapidement exclus. Il arrive que la présence des pairs soit préalablement convenue avec les animateurs, qui ne pouvaient placer tout le monde à la tribune, ou souhaitaient quelque peu artificiellement engager le débat. Ce peut être aussi l'effet de logiques structurelles, dans le cas de colloques, cycles, etc. Les pairs peuvent enfin faire planer sur le débat la menace de leur désapprobation: celle-ci se montre au mieux par leur silence, ou pire, par leur départ en cours de débat. Il ne peut presque rien arriver de pire à un animateur, sauf de ne pas connaître les pairs, et de ne pas leur donner la parole, ou de la leur donner sans les appeler par leur nom.

\section{L'intervention quantitative du public}

Bien évidemment, celle-ci est fonction de la nature du débat. La variable principale en est souvent moins la difficulté effective, ou la technicité du débat, que la thématique. Si les débats d'idées, notamment philosophiques, rassemblent toujours un vaste public, les débats esthétiques, en présence d'un créateur, sont ordinairement beaucoup plus confidentiels. L'animateur joue aussi sur la présence ou l'absence de "vedettes », pour attirer ou pas un public important. Le nombre n'est pas une variable absolue : il influe sur la dynamique du débat en fonction de la taille de la salle. Ainsi, cinquante personnes seront un public nombreux à la Salle d'actualité, dérisoire dans la grande salle. Aussi le nombre des personnes n'est-il qu'un indicateur relatif du succès ou de l'échec d'un débat : tel débat confidentiel avec un créateur inconnu sera un succès s'il a réuni une dizaine d'amis. Tel autre, à grand renfort de vedettes médiatiques, verra son échec dans le fait de n'avoir pu mobiliser qu'une trentaine de personnes. Mais un public trop important peut aussi faire échouer un débat (le cas de figure est moins grave dans le cas de la petite salle ou de la grande salle qui ferment leurs portes) : il engendre en effet va-et-vient, bruits de fond, frustrations. Toutefois, en tenant compte de ces remarques, le nombre du public est un indicateur de la place des débats dans le champ culturel (cf. chapitre 4, l'exemple de l'histoire et de la philosophie).

\section{Quand un débat peut-il être dit réussi ?}

Il n'existe pas de critères objectifs et intangibles de la réussite d'un débat. Celle-ci sera donc fonction d'appréciations subjectives. Toutefois, ces appréciations sont aussi fonction de la place qu'occupent les intervenants dans le débat et de leurs attentes. L'idéal serait de pouvoir mesurer l'indice de satisfaction du public. Mais outre que nous sortirions des limites de cette étude, une telle mesure poserait des problèmes méthodologiques considérables. Sans compter que ce n'est là qu'un paramètre parmi d'autres : il peut bien y avoir des débats où l'opinion du public ne compte pas, ou peu. En revanche, la représentation de la satisfaction du public joue un rôle important dans l'appréciation qu'organisateurs et participants portent sur la réussite du débat.

Les organisateurs ont structurellement tendance à considérer l'affluence comme un critère de réussite. C'est bien entendu justifié, à condition de tenir compte des limitations évoquées plus haut. Le critère de l'affluence peut d'ailleurs jouer dans des sens différents : tel qui parvient régulièrement à organiser des débats très spécialisés avec un public restreint, mais très satisfait, se verra taxer d'élitisme, tandis qu'un autre qui remplit des salles pourra être soupçonné de démagogie. 
La réussite peut aussi s'apprécier du côté des participants. Ceux-ci, s'ils n'adoptent pas, ce qui est fréquent, le ton blasé de l'invité contraint mais courtois - « Moi, vous savez, les débats... J'en fais tellement... Ça n'apporte pas grand'chose... Mais enfin... »-, qui en fait masque rarement un narcissisme diffus mais réel, ont tendance à apprécier le débat d'après ce qu'ils perçoivent de leur propre intervention: ont-ils le sentiment d'avoir été «bons », et le débat est pour eux réussi ; se croient-ils au contraire victimes d'une mauvaise forme (ou de la malignité d'un autre intervenant, ou de l'animateur), et le débat aura été franchement désastreux. Rares sont ceux qui peuvent s'exclamer : "Je n'ai pas dit ce que j'avais à dire, mais j'ai trouvé le débat fort instructif. » Il y a toutefois d'heureuses exceptions, de plus en plus fréquentes évidemment à mesure qu'on s'écarte des participants à forte notoriété : les intervenants alors, non seulement sont ravis d'avoir été invités, mais souvent aussi d'avoir ainsi pu faire la connaissance de tel ou tel autre participant. On ne peut en général obtenir d'eux une appréciation « objective » quant à la réussite du débat.

L'écho médiatique est aussi un indice: chacun jugera réussi un débat dont l'écho médiatique sera satisfaisant. Mais là aussi la considération est faussée par le fait que cet écho est largement le fait de la préparation du débat, plus que de son déroulement luimême. Les débats organisés par la BPI et Le Matin de Paris se virent chaque fois gratifiés d'un long article dans Le Matin de Paris : ce n'est pas leur faire injure que de penser que d'autres débats moins couverts par la presse ont pu être tout aussi intéressants.

La représentation du public est aussi un élément déterminant: "les gens sont contents ", phrase justifiée par des bribes de conversations saisies au vol, ou même rituellement prononcée, montre qu'il est nécessaire de la prendre en compte. Mais elle est totalement protéiforme.

On pourrait être tenté de trouver des critères objectifs dans la mesure de l'impact du débat: impact d'une présentation de livre sur les ventes de l'ouvrage, etc. Mais il semble bien que le débat ne vienne ici renforcer ou accompagner un mouvement qu'il est en lui-même incapable de susciter. Le discours des attachées de presse sur l'influence des débats à la Salle d'actualité est à cet égard éloquent : le débat ne peut pas faire décoller un livre qui ne marche pas. Et s'il marche, le débat n'est que l'un des éléments d'amplification du succès. Elles continuent toutefois, de manière paradoxale, d'assiéger la Salle d'actualité pour obtenir des débats. En fait, il vaut mieux lire cette relation en sens inverse : la plus ou moins grande notoriété d'un auteur va configurer le débat, en le polarisant d'une certaine manière.

51 Cette relativité, voire cette indétermination de la réussite est ici le signe d'une ouverture du débat sur le champ culturel dans son ensemble: selon que l'on considérera le débat dans son instantanéité, sa performance propre, ou en fonction de son inscription dans le champ, l'avis pourra être différent, pour le même observateur (ou participant). Ainsi un débat peut être jugé catastrophique dans son déroulement, parce qu'on s'y est ennuyé, ou parce que «les vraies questions n'ont pas été posées ». Mais il pourra en même temps donner l'impression d'avoir un peu déplacé des configurations trop stables. Inversement, tel débat parfaitement huilé dans son déroulement peut n'être que d'un médiocre apport à une transformation du champ. C'est donc vers celui-ci qu'il nous faut maintenant nous tourner. 


\section{Chapitre 4. Les débats du Centre dans le champ culturel}

1 La place des débats organisés au Centre Georges Pompidou dans l'ensemble du champ culturel ne se laisse pas facilement cerner. A cela trois raisons majeures : tout d'abord, on l'a vu, la variété des débats organisés au Centre ne permet guère une étude sur un corpus homogène. On peut s'attendre à ce que cette place varie en fonction du type de débats considéré. La seconde raison plaide à vrai dire contre la première. C'est qu'il y a un «effet Beaubourg» qui tend à constituer le Centre Georges Pompidou en un tout indifférencié, mais où cette fois-ci les débats ne sont qu'une des activités du Centre, parmi d'autres. La première raison manque la question par défaut, la seconde par excès, pourrait-on dire. Enfin il y a une troisième raison qui tient à la difficulté d'appréhender le champ culturel en lui-même. Comment le définir? Quelles institutions privilégier?

2 Ces difficultés principielles n'ôtent cependant pas toute pertinence à la question. En effet, les débats du Centre sont bien en rapport avec un champ plus vaste, qui les englobe et dont ils constituent une des articulations. Il semble que la meilleure approche consiste à tenter d'inventorier les fonctions que joue le Centre dans ce champ culturel, soit spécifiquement, soit à l'appui d'autres courants qui parcourent le champ. Nous pouvons nous autoriser à parler de champ, malgré la difficulté signalée plus haut, dans la mesure où il est loisible de se fonder sur la représentation intuitive que chacun en a, attestée par la circulation constante entre institutions, individus et productions qui forment ce champ. De la presse à l'Université, en passant par diverses institutions dont le Centre, une circulation incessante de thèmes, de questions, de personnes a lieu en permanence. Nous n'appellerons champ que ce cadrage minimal, excluant ainsi d'emblée toute possibilité de lui assigner des limites définies et une normativité propre. L'hypothèse est plutôt qu'un tel champ fonctionne comme un terrain-ouvert, sujet à de constants remaniements de limites, et qu'en lui coexistent des modes de classement, des manières de hiérarchiser les problèmes ou les personnes fort diverses. Pour prendre deux exemples extrêmes : la télévision et l'Université, il est clair que ces deux institutions font partie du champ culturel. Pourtant, leurs modes respectifs de fonctionnement ne se recoupent pour ainsi dire jamais, leurs hiérarchies sont souvent contradictoires, leur antagonisme profond. A la limite, la télévision ne vise qu’à 
produire un divertissement à grande échelle (ou en tout cas se refuse avec énergie à assumer la moindre fonction pédagogique, $c f$. les déclarations de la plupart des professionnels, chaînes privées et publiques confondues), pour lequel les productions propres au champ culturel ne sont retenues que comme un élément parmi d'autres. Inversement, l'Université possède une normativité interne propre, fondée sur l'ordre du savoir, et ne se rapporte au champ culturel que par sa frange "publique»: production de livres, de revues, de thèmes et de questions. La recherche et l'enseignement qui sont ses principales fonctions tendent à y échapper par définition.

C'est aussi ce qui permet de prendre en compte les deux premières difficultés : elles ne sont consistantes qu'à refuser d'admettre la labilité propre au champ culturel. Si on l'admet, on peut au contraire moduler les interventions du Centre dans le champ selon les débats, mais aussi selon l'état du champ à un moment considéré. Quant à la globalité de «l'effet Beaubourg ", elle joue aussi son rôle dans la structuration du champ. Mieux : à cette aune, le Centre paraît être un lieu d'observation privilégié quant à la structure et au mode de fonctionnement de ce champ culturel. En effet, moins réductible que d'autres institutions à des fonctions uniques ou du moins principales, il en cumule plusieurs, et les conjugue de manière spécifique. Il échappe par là même au stéréotype dans lequel ira trop facilement se mouler la télévision ou l'Université, ainsi que le discours sur ces institutions. Il sollicite une réévaluation de l'analyse de la logique qui informe l'espace public.

4 Aussi, dans un premier temps, est-il nécessaire d'envisager la place des débats du Centre dans le champ culturel sous l'angle des fonctions qu'ils remplissent. On peut distinguer trois grandes fonctions: une fonction de diffusion, une fonction de légitimation, une fonction de production. Ce n'est pourtant pas céder à une tentation «fonctionnaliste » : l'entrecroisement et le recouvrement partiel des fonctions mises en évidence appelle un deuxième moment de l'analyse, qui se centre davantage sur les logiques qui règlent le champ culturel et l'espace public en général.

\section{Les fonctions du Centre dans le champ culturel}

\section{La fonction de diffusion}

5 On pourrait appeler débat-relais celui qui remplit cette fonction : conçu pour relayer une initiative extérieure et antérieure au débat, celui-ci est en quelque sorte une chambre d'écho, et ne prétend pas innover dans le champ culturel. Il vient en appui à autre chose, livre, exposition, événement artistique qu'il a pour fonction d'éclairer et de mettre en valeur. C'est aussi qu'il est un intermédiaire entre cette « œuvre » et un public potentiel. Ce débat s'accommode de la configuration du champ et se modèle sur elle. C'est le cas de figure le plus répandu (pas seulement pour les débats du Centre). Le modèle pourrait en être les débats de la BPI autour de livres (Salle d'actualité). Mais, même dans ce cas, il peut ne pas s'agir de routine : le débat peut repérer des grandes tendances ("Les biographies historiques », BPI, 12 février 1987), s'inscrire comme un moment dans le développement d'un « débat » public plus large («La pensée 68 », 23 janvier 1986, qui fait intervenir de nouveaux participants : François de Négroni, Pierre Assouline, Pierre Nora; $c f$. aussi la «Revue parlée » sur les nouveaux philosophes), ou même lancer un débat sur un thème (rencontres autour d'éditeurs: BPI, février-mars 1977, la culture scientifique et son public, 23 mars 1978, ou la traduction, BPI, 7 
décembre 1978). Il est ainsi l'un des rouages de la diffusion de l'«œuvre » dans le champ culturel, sans d'ailleurs que l'on puisse toujours en mesurer les effets spécifiques. La fonction propre du Centre est ici limitée: nombre de ces débats pourraient avoir lieu ailleurs (Fnac, France-Culture) : il arrive d'ailleurs que ce soit le cas, d'une part, parce que certains débats du Centre sont enregistrés et retransmis sur France-Culture (ou parfois aussi sur Radio-France internationale); d'autre part, parce que certains thèmes, certains auteurs, certains livres peuvent donner lieu à des débats similaires au Centre et à la radio à quelques semaines d'intervalle. En revanche, il n'est pas négligeable pour le Centre que de tels débats y soient organisés. Ils contribuent à figurer celui-ci comme un acteur important de la diffusion culturelle: en témoigne le souci des attachées de presse des maisons d'édition, par exemple, pour qui «obtenir » un débat en Salle d'actualité est aussi important que de voir paraître un article dans un journal, malgré l'aveu du peu d'impact des débats en tant que tels sur les ventes de livres. Peut-être s'y glisse-t-il quelque chose du prestige général du Centre qui retombe sur toute activité qui y trouve sa place. Là, la fonction de diffusion vient frôler la fonction de légitimation.

6 Il est un cas particulier de la fonction de diffusion : quand le débat vient relayer une initiative interne au Centre : expositions, par exemple. En fait, il s'insère alors d'emblée dans un ensemble, dont l'exposition comme les débats sont des moments. D'un certain point de vue, le débat est pourtant bien en posture de médiation par rapport à l'exposition: il en met en scène les intentions, les prolongements, les attendus. Cette vertu pédagogique, souvent thématisée comme telle (les débats du MNAM liés aux expositions) est néanmoins paradoxale, car les expositions, surtout les grandes expositions communes à tous les services du Centre, voient défiler plus de monde qu'aucun débat et même tous les débats réunis n'en verront jamais. Là, la diffusion est plutôt intensive qu'extensive pourrait-on dire, et à la limite le rapport pédagogique peut s'inverser : l'exposition est alors la propédeutique du débat, l'étape première et obligée qui doit mener à celui-ci. Il n'est pas exclu que de telles stratégies soient parfois à l'œuvre. En revanche, l'hypothèse d'un débat destiné à la promotion de l'exposition constituerait un cas particulier, à vrai dire peu fréquent, de la fonction de diffusion.

\section{La fonction de légitimation}

7 Elle prend appui sur l'image du Centre prise globalement. En effet, pour un livre, pour un thème, pour un individu (auteur ou non), être évoqué dans le cadre d'un débat du Centre équivaut à une forme de consécration. Celle-ci est bien évidemment différente selon la légitimité déjà acquise ou non, le type du débat, la nature des (autres) intervenants. Mais plusieurs organisateurs de débats l'assurent: on n'a jamais vu quelqu'un refuser d'intervenir dans un débat du Centre sauf pour raisons d'emploi du temps, bien entendu, dont on sait qu'elles peuvent être la forme courtoise du refus; cependant, deux exemples d'intervenants parmi les plus consacrés par ailleurs, donc les plus légitimes et les plus susceptibles de ne trouver aucun gain symbolique supplémentaire à une intervention au Centre confirment le propos: leur refus n'était pas fondé sur des prétextes mais sur de réels empêchements. Or cet engouement n'a pas de motifs économiques : la médiocrité, sinon l'absence totale de rémunération des intervenants (au demeurant relative, comparée aux autres institutions du champ: radio, ou même Université) interdisent de le penser. On peut faire l'hypothèse que toute tribune est bonne à prendre et constitue, à l'égard des positionnements respectifs 
dans le champ, un atout supplémentaire (par exemple en termes d'accumulation de capital symbolique), ou que les intervenants sont à l'affût de toutes les occasions possibles de délivrer un «message ", ou du moins de défendre leur point de vue (selon qu'on se rallie à la version démystificatrice ou au contraire prédicatrice de l'hypothèse). A cet égard, les débats du Centre ne disposeraient d'aucun privilège, et seraient tout simplement aussi « courus » que toute autre manifestation dans le champ. C'est le fonctionnement d'ensemble de celui-ci qui devrait expliquer un tel succès, ou tout simplement la bonne volonté des intervenants qui en général accepteraient toutes les invitations d'où qu'elles viennent.

8 Sans nier le bien-fondé de cette hypothèse d'ensemble, qu'on peut d'ailleurs rapporter au mode de fonctionnement de l'espace public démocratique, c'est-à-dire au règne de l'opinion, lequel veut que quelque chose (i.e. un livre, une "œuvre », une question, un individu) n'« existe » que si l'on en parle (cf. deuxième partie de ce chapitre p. 73 et suivantes), il faut cependant se rendre à l'évidence d'une spécificité du Centre à cet égard. Si un débat au Centre n'est jamais qu'un débat de plus dans une gamme de débats possibles, la forme de ce débat est quant à elle bien spécifique. Débat devant un public physiquement présent, à la différence du débat radiophonique ou télévisé, il est cependant débat devant un public "large ", à la différence du débat organisé par une association, une instance universitaire ou para-universitaire pour un public ciblé (dans le cas général : certains débats du Centre peuvent cependant reproduire cette situation en son sein). Le point de comparaison le plus proche, mais qui mettra aussi en évidence la spécificité du Centre, serait les débats organisés par la Fnac: le public y est sensiblement le même (pour la Fnac de Paris), l'affluence aussi, les thèmes sont souvent voisins, les animateurs peuvent être communs et les intervenants aussi. Mais le prestige symbolique du Centre est néanmoins supérieur (ce que compense la Fnac par l'octroi d'une rémunération matérielle plus importante, au moins pour les animateurs). Il tient à plusieurs facteurs : d'une part, le prestige qui s'attache en France à toute institution publique, rehaussé ici par la légitimité montante des institutions de la Culture; mais d'autre part, le Centre dispose d'une image de modernité, de pluridisciplinarité et de médiateur avec un large public qui pris ensemble en font l'originalité. Un nom la condense : «Beaubourg ».

9 Modernité : c'est d'abord la modernité architecturale du Centre, sans doute le premier monument d'art contemporain à avoir été adopté par la ville de Paris. C'est bien sûr le MNAm et l'IRCAM. C'est aussi un souci constant des débats. Pour autant, ce souci de la modernité a su intégrer les critiques du modernisme et progressivement rompre avec les tentations avant-gardistes datant de l'utopie du Centre. Sans créditer le Centre du bénéfice de cette mutation, force est de constater que l'effritement des thématiques avant-gardistes dans le champ culturel a coïncidé avec sa venue à maturité.

10 Pluridisciplinarité : inscrite dès le début dans la vocation du Centre, elle est à elle seule un facteur de légitimation culturelle, dans la mesure où elle met en scène les effets de fécondation (effectifs, supposés ou attendus) d'une discipline envers une ou des autres. Ce n'est qu'ultérieurement qu'on peut retrouver des débats portant sur l'évaluation critique de ce qui se loge à l'enseigne des pluri, inter, ou transdisciplinarités. Celle qui s'effectue à Beaubourg, sans doute mal définie conceptuellement, peut pour cette raison même en figurer l'énigme. Plus encore que l'exposition, congénitalement affligée par le privilège du voir ( $c f$. Les Immatériaux), le débat est le lieu de cette pratique de confrontation disciplinaire. En témoigne par exemple la distance entre le séminaire 
«Matière et philosophie» qui accompagna à l'Espace séminaire l'exposition Les Immatériaux, et ce, malgré la présence conjointe de Jean-François Lyotard au principe de l'exposition et au cœur du séminaire. De fait, la confrontation entre chercheurs d'horizons de pensée et de disciplines divers eut lieu dans le séminaire.

11 Médiation avec le public: c'est là le motif qui évite à la modernité et à la pluridisciplinarité de se perdre en querelles académiques. Mais c'est aussi la reconnaissance d'une légitimité, puisque ce qu'on présente au public dans le cadre du Centre a été jugé digne de l'être. Certes, ce premier procès en légitimation est soumis à des instances d'appel, dont les réactions dudit public, celles des pairs, celles d'autres institutions sont autant de points d'ancrage. Néanmoins, si l'on fait l'hypothèse que toute apparition en public élève par là même une prétention à la légitimité, le fait de figurer au sommaire d'une des activités du Centre est une confirmation considérable de cette prétention.

12 L'entrecroisement de ces trois motifs figure donc l'originalité du Centre (seule peutêtre une institution comme La Villette pourrait rivaliser avec lui sur ce point ; mais elle est trop récente pour pouvoir supporter une comparaison instructive, et de toute manière échoue à mobiliser aussi massivement la légitimité proprement « culturelle »). De ce point de vue, toute manifestation qui s'y déroule se voit dotée d'une légitimité propre. La fonction de légitimation culturelle n'y est jamais assoupie. Qu'en est-il spécifiquement des débats? Ceux-ci sont, on l'a vu, pris entre les deux pôles de référence que sont le colloque universitaire et l'émission de télévision style Apostrophes. On sait aussi que nombre de débats du Centre peuvent être assimilés à l'un ou à l'autre de ces deux pôles (cf. chapitre 2). Mais la majeure partie d'entre eux sont de nature intermédiaire. Or il est loisible de considérer le premier comme l'exemple d'une manifestation à légitimité dérivée: c'est en raison d'une légitimité antérieure que la manifestation elle-même est légitime, même si elle vise bien entendu à opérer une modification dans le champ des légitimités reconnues. De ce point de vue, il est symptomatique que le colloque universitaire a plutôt tendance à affecter le champ de la légitimité des objets ou des thèmes que celui des individus. A l'inverse, l'autre pôle sera dit de légitimité postulée : l'émission de télévision est le cadre où va se manifester une prétention à la légitimité, qui attend une confirmation. La performance y est exigée, bien plus que la compétence réelle ou supposée (le cas bien connu de la contreperformance pouvant d'ailleurs entrer dans une stratégie complexe de performance : c'est bien ce qu'illustre la figure légendaire de Patrick Modiano dont la célébrité vient de ses bafouillements à Apostrophes). Là, l'objet est secondaire et l'individu passe en première ligne. Bien entendu, une telle distinction n'est valide qu'à la limite : la participation à un colloque universitaire peut être aussi l'occasion d'une première avancée sur la scène, d'une "prise de parole " inédite; inversement, le passage à Apostrophes suppose au moins le préalable de l'écriture d'un livre. Néanmoins, il est permis de suivre Bernard Frank lorsqu'il constate: "Auparavant, nous autres écrivains, nous n'avions qu'un écrit à passer; désormais il nous faut aussi passer un oral $^{1}$ ( " A filer la métaphore, on est amené à concéder que l'épreuve d'admission compte donc davantage que celle d'admissibilité, préalable obligé certes, mais qui n'est en rien décisive. Au demeurant, à se pencher ainsi sur les modalités de validation des légitimités, on est conduit à grandement relativiser les analyses de Pierre Bourdieu qui supposent une échelle fixe et établie des légitimités ( $c f$. deuxième partie de ce chapitre p. 73 et suivantes). 
13 Dans le cadre des débats du Centre, la rémanence de la légitimité dérivée reste forte, en raison même de la provenance universitaire de nombreux acteurs (cf. chapitre 3). Mais faut-il exclure toute légitimité postulée ? Non seulement c'est le cas pour un certain nombre d'acteurs (cf "L'impétrant ", chapitre 3), même dans le cas où le débat luimême se rapproche le plus du modèle universitaire du colloque, mais la forme même du débat tend à l'induire. Le débat au Centre, s'il est permis ici de réduire les différences valorisées ailleurs, se situe dans un entre-deux qu'on qualifierait volontiers de légitimité opérée : le débat est en lui-même mise en œuvre de cette légitimité, il condense dans son espace dérivation et postulation, il est le lieu d'accomplissement de la prétention à la légitimité. Par lui la compétence se fait performance. Certes, le débat n'est pas le seul à opérer sur ce mode : l'écriture d'un livre, la réponse à une interview, la délivrance d'un enseignement, la conduite d'une enquête le font aussi ; mais il est sans doute le seul à opérer principalement sur ce mode et à la limite, exclusivement. Ce n'est pas dire que la légitimité opérée se confond avec la légitimité postulée : celle-ci peut échouer radicalement, tant la performance individuelle y est prégnante. La prétention y est considérable due au nombre de téléspectateurs, ce qui vaut dans une moindre mesure pour les auditeurs du débat radiophonique; due aussi, paradoxalement, au surinvestissement dont bénéficie l'animateur : son choix attend d'être validé par la qualité de la prestation. Le débat partage plus équitablement la prétention, et donc, s'il la sollicite, équilibre la performance par la compétence. Or ce trait du débat, qui affecte tout débat, ceux du Centre le manifestent éminemment. D'être au carrefour de légitimités cumulées les qualifie à cet effet. Se confirme ainsi ce que nous constations de la fonction de diffusion : elle tend vers la légitimation. On peut dire que la fonction de légitimation, sous la forme de la légitimité opérée, affecte à des titres divers tous les débats du Centre.

\section{La fonction de production}

Peut-on aller plus loin, et supposer que le Centre, par les débats, est un lieu de production dans le champ culturel, confirmant ainsi pour leur part un aspect de sa vocation, «favoriser la création des œuvres de l'art et de l'esprit » (loi du 3 janvier 1975 définissant les missions du Centre) ? Si l'on a préféré ici le terme de production à celui, plus connoté, de création, c'est qu'un débat pourrait être à grand peine qualifié de création. En outre, l'inflation des recours à la rhétorique de la création dans le champ culturel aurait menacé de dissoudre la spécificité des débats. Mais répondre à la question exige aussi que l'on s'émancipe d'une trop grande coupure entre légitimation et production. Le fonctionnement du champ culturel n'est pas tel que l'on puisse y repérer des lieux de production pure, préalables et antérieurs aux lieux de diffusion et de légitimation. A conserver l'analogie économique désormais classique pour évoquer ces questions, le marché n'est pas sans interférer sur les formes et les modes de la production de biens matériels. Mais cette analogie elle-même rencontre ses limites quand on prend en compte le système des normes qui régissent le champ: elles régissent la production, mais pas au point de l'empêcher de tester des évolutions, de suggérer des adaptations, de tenter des subversions. La légitimité des normes est ainsi sans cesse en mouvement, et soumise aux réélaborations que lui fait subir la production, qui ne cesse pourtant jamais d'être induite par ces normes. C'est pourquoi nous avons préféré parler de fonctions plutôt que de lieux, celles-ci se prêtant à la superposition en un même lieu. L'espace de jeu ainsi défini circonscrit une gamme de 
pratiques qui ont précisément pour enjeu ces remaniements de légitimité. Le débat est l'une de ces pratiques, et on peut penser qu'il n'échappe pas, parfois, au cumul des fonctions.

On peut parler à cet égard de débats dont la fonction est inaugurale, au sens où ils parviennent à imposer un thème, une problématique, un remaniement du champ. De tels débats s'insèrent souvent dans un cycle, ou prennent la forme du colloque, bien que cette structuration plus forte ne soit pas indispensable à l'inauguration engagée par le débat. Quatre exemples peuvent être ici convoqués, qui permettront de mieux cerner les modalités de l'articulation des deux fonctions.

\section{Premier exemple : le cas des journées de cultures juives méditerranéennes et orientales (12-15 septembre 1980)}

16 Il y avait eu fin 1979 des journées de la culture yiddish organisées dans le cadre de la «Revue parlée ». En 1980, une génération s'est écoulée depuis l'émigration des Juifs d'Afrique du Nord et d'Égypte. Ces derniers avaient commencé à reconstituer leur patrimoine et leur mémoire. 1975 : création d'« Identité et dialogue », association des Juifs marocains. 1978: association des Juifs d'Égypte. Il commençait à y avoir un décloisonnement des communautés orientales. Elles étaient à la recherche d'une identité. Mais personne n'avait encore écrit sur la « question sépharade » en France.

Selon le propre récit des organisateurs, ils décident donc sans projet précis de monter dans les locaux du Centre Georges Pompidou des journées qui permettent une expression des cultures juives orientales qui ne soit ni religieuse, ni folkloriste. « Notre projet, c'était de présenter une culture qui nous tenait à cœur ", commente un des initiateurs. Ils demandent au Centre d'être le coup d'envoi de quelque chose qui n'avait pas émergé comme culture légitime. Ils ont dû déjouer la défiance de l'institution puis faire avec l'opposition du Grand Rabbinat, quand on découvrit que la manifestation débutait le deuxième jour du nouvel an juif. Ce qui donna raison aux organisateurs, tous laïcs, fut l'immense succès de ces journées pendant lesquelles les deux salles et les couloirs du sous-sol, envahis par la foule, ne désemplirent pas.

La manifestation culturelle proposa, autour d'une exposition de photos et d'objets, de la projection de films, des conférences sur l'histoire de juifs méditerranéens et orientaux, des rencontres d'écrivains, de femmes, des tables rondes politiques. Elle donna lieu à une publication, les organisateurs ayant conscience que leur groupe n'aurait pas de lendemain et qu'il fallait prendre date.

Mais les retombées de ces journées furent immenses. Il y eut d'abord une répercussion immédiate: la première page du Monde, une page du Matin de Paris leur furent consacrées. Mais surtout, la présence au Centre Georges Pompidou « donna pignon sur rue aux cultures juives sépharades ", leur conférant une légitimité tout en jouant un rôle initiateur. Les conséquences en furent la publication de livres (Les Juifs du Nil, Les Juifs de Tunisie, Les Juifs d'Algérie), la création d'associations culturelles et de radios juives qui ont pris leur essor avec des animateurs issus de ces journées, quand la loi sur les radios libres fut adoptée.

20 Le choix du Centre apparaît comme fondamental dans le phénomène de résonance de la manifestation. Lieu neutre et prestigieux, il a donné à ces journées un statut en dehors des instances de la communauté juive. Les cultures présentes ont acquis d'abord une reconnaissance sur la place publique. Puis, il y eut un effet en retour à l'intérieur de la 
communauté. La fonction inaugurale du Centre a ici joué à plein, sans qu'il soit toutefois possible d'isoler le rôle des débats au sein de ces journées. Tout au plus peuton faire remarquer que l'enjeu ne pouvait que mobiliser un certain nombre d'arguments, et qu'ils furent plaidés dans les débats.

\section{Deuxième exemple : Vienne, ou la construction d'une thématique}

21 On dispose là d'un indicateur très précis du début de la "mode viennoise »: le numéro de la revue Critique intitulé «Vienne début d'un siècle » (1975). Puis très vite vont se multiplier les ouvrages. Mais le Centre relayera constamment ce travail dans le cadre de la «Revue parlée » (début des années quatre-vingt). D’autres cycles, par exemple Musil à la Bpi, s'inscrivent dans cette problématique. Des uns aux autres, la présence de participants communs (par exemple J. Bouveresse) accrédite l'idée d'un «objet» consistant. Bien entendu, tout cela n'est pas sans faire écho au Centre d'intérêts plus larges, venus des champs de la recherche universitaire (intérêt pour le Cercle de Vienne, l'architecture de Loos), de l'édition (republication des œuvres de Schnitzler, traduction du livre de Schorske), de l'esthétique (la peinture de Klimt), de la critique (la référence à Kraus, à Musil), de la pensée politique (l'empire austro-hongrois et la double monarchie), ou plus généralement de questions insistantes dans le champ culturel (la psychanalyse et l'enjeu de l'œuvre de Freud, le destin des juifs d'Europe centrale). Une configuration se dessine ainsi, qui sollicite d'autant plus l'attention qu'elle est pluridisciplinaire.

22 C'est donc tout naturellement en un sens que viendra la grande exposition Vienne, avec tout un cortège de débats qui approfondiront les perspectives (et là encore la « Revue parlée » avec le colloque dirigé par F. Kreissler), et qui verra le couronnement de cette «mode viennoise ». Cette consécration ne pouvait qu'aboutir au Centre : le caractère pluridisciplinaire de l'objet s'y prêtait particulièrement, et celui-ci avait depuis longtemps manifesté pour Vienne un intérêt soutenu.

Sur ce fonctionnement, on voit comment une thématique émerge et comment le Centre est à la fois relais, amplificateur, consécrateur, catalyseur. Une interaction constante entre ses activités (notamment les débats) et le champ culturel est venue tisser la possibilité d'accueillir une grande manifestation, qui vient définitivement consacrer l'objet « Vienne » comme objet culturel légitime.

\section{Troisième exemple : les cycles « Fabrication du corps humain et droits de I'homme » à la BPI}

Là, il s'agit moins de l'invention d'une sensibilité que d'affirmer un domaine de recherche et de préoccupations. Mais le cycle lui-même devient productif puisque les actes en sont recueillis en livre'2. En fait, on assiste à l'invention d'une problématique, qui ira en s'affermissant et qui rencontre les interrogations éthiques, politiques et philosophiques posées par la biologie contemporaine. Que cette quête ait été placée sous l'égide d'une réflexion sur les droits de l'homme n'est pas ici sans signification : ceux-ci sont à la fois le «mot d'ordre » qui signale l'originalité de la démarche et le moyen de fournir au public un point de repère parmi les enjeux théoriques et médiatiques du temps.

Le cycle, après une première année de fonctionnement, donnera lieu à un prolongement, qui est à la fois un approfondissement et un déplacement : sans cesser 
d'articuler des débats, on s'oriente quand même de plus en plus vers une formule de type séminaire, où des participants réguliers ont le sentiment de faire un travail en commun. Des journées pourront alors être organisées qui consacrent la place acquise par la question et aussi le mode de fonctionnement. La production d'un livre à miparcours a aussi beaucoup fait pour consacrer ce type de travail, mais on peut penser que l'existence ailleurs dans le Centre de structures vouées à ce type de recherches ("Espace séminaire », cf. plus loin p. 72) n'a pas peu accrédité l'idée qu'une recherche était possible au Centre, excédant les cadres universitaires classiques.

\section{Quatrième exemple : Le colloque « L'enfant, la famille et l'État » organisé par la cellule Histoire et société}

26 Avec ce colloque, organisé par la cellule Histoire et société à la suite d'une réflexion sur

le bicentenaire de la Révolution et la manière dont elle pouvait s'y intéresser, on en vient à une structure qui est quasi intégralement celle de la recherche scientifique universitaire. Les thèmes retenus, la nature des intervenants, presque tous chercheurs ou professeurs, le style choisi (colloque, et même colloque fermé avec inscription payante) le signalent clairement. On peut même aller plus loin, en constatant que parmi les diverses manifestations auxquelles a donné lieu le bicentenaire, celle-ci était l'une des plus novatrices intellectuellement. Faut-il en conclure que le Centre n'a plus alors de spécificité ? Il est vrai qu'une telle manifestation peut tendre à n'utiliser le Centre que comme lieu d'accueil, au même titre que n'importe quel autre. On peut toutefois remarquer que le caractère délibérément pluridisciplinaire du colloque, qui associe historiens, sociologues, juristes et philosophes n'aurait pas manqué de buter sur les corporatismes universitaires, s'il avait dû se faire ailleurs. Significative est à cet égard la collaboration avec le Centre de recherche interdisciplinaire de Vaucresson, dont l'insertion dans le champ institutionnel de la recherche est difficile. Là encore, des marges de la recherche et de l'université proviennent des initiatives qui peuvent trouver au Centre leur aboutissement.

Il faut ajouter à ces exemples, significatifs mais qui recensent des actions plus ou moins ponctuelles, les modalités plus diffuses et plus discrètes de deux autres types de fonctionnement : celui de l'Espace séminaire, et celui du cycle de conférences organisé par Yves Michaud au MNAM. Dans le premier cas, on a une structure dont la vocation explicite est de travailler à l'émergence de problématiques nouvelles, interdisciplinaires, quoique fortement marquées au coin de la pensée philosophique. Dans le second au contraire, une pédagogie de haut niveau sous la forme de conférences d'approfondissement culturel. Un séminaire, une série de conférences: c'est le « troisième cycle » des cursus du Centre.

L'Espace séminaire joue sur une frontière entre les activités du Centre, qu'il relaye pour sa part une fois par an, et un souci propre de recherche, lequel se donne des thèmes spécifiques: l'imaginaire de la Grèce antique, par exemple. Ses modalités propres de fonctionnement le conduisent à conjoindre le séminaire fermé et le débat public (avec en outre une variante plus pédagogique dans le cadre des activités de la cellule Liaisonadhésion). Le haut niveau de légitimité acquis par la plupart des chercheurs invités n'exclut pas une certaine ouverture, laquelle se fait en deux directions: vers des chercheurs plus jeunes, et dans une dimension internationale, qui est un des soucis constant de l'Espace séminaire. L'inscription dans le champ se joue essentiellement sur ces deux points : accorder une légitimité provisoire et présumée à des plus jeunes, et 
offrir aux étrangers reconnus la possibilité d'une audience en France. Ce fut notamment le cas pour les philosophes italiens invités, Gianni Vattimo et Alessandro dal Lago, par exemple, qui n'étaient pas traduits en français lors de leurs interventions à l'Espace séminaire et le furent ensuite. Non que l'espace ait une fonction de pur révélateur : mais il contribue fortement à la reconnaissance en cours de la validité d'une œuvre ou d'une pensée. Le succès enfin des débats publics qui concluent les séminaires fermés, mais en fait en reprennent le plus souvent les éléments, tient en grande partie à cette répétition : avertis de leurs discours respectifs, les participants ont tendance à en présenter les arêtes les plus vives. Il faut aussi souligner que se confirme ici un trait déjà relevé par ailleurs: le succès des débats à coloration philosophique.

29 La fonction des conférences du MNAM est tout autre : il ne s'agit pas de produire du savoir, ou des idées, mais de mettre en scène quelques-unes des réflexions les plus novatrices dans le champ de la recherche esthétique et critique contemporaine. Aussi ces conférences prennent-elles l'allure de véritables cycles de formation, qui sont en effet assez loin du débat proprement dit, mais n'en constituent pas moins une des modalités privilégiées de la parole publique au Centre. Là le Centre est un médiateur, dans le meilleur sens du terme, et contribue ainsi à la diffusion de recherches parfois difficiles. Mais il produit la possibilité de voir se succéder dans le même cycle des travaux de premier plan. A noter que la dimension d'ouverture à l'étranger est ici aussi très prégnante. Certains cycles, comme celui consacré à la muséologie, n'ont pas eu d'équivalent ailleurs.

30 On voit donc, à parcourir ces différents cas de figure, que le Centre articule constamment, les unes aux autres, les fonctions de médiation/diffusion, de légitimation et de production. En ce qui concerne plus particulièrement cette dernière, il est désormais reconnu comme un lieu qui n'est pas seulement d'échange, ni d'exposition, mais d'élaboration pleine et entière. Marginal par rapport aux grandes institutions censées légitimement produire de la culture (et d'abord, en ce qui concerne les débats, l'Université), le Centre est en position de pouvoir infléchir le champ, de déterminer des glissements de légitimité. Il lui est ainsi possible de faire apparaître, de rendre visible dans l'espace public des thèmes nouveaux et aussi de servir la stratégie de nouveaux acteurs. Inversement, il sait aussi à l'occasion se légitimer en empruntant au champ questions et intervenants, de manière à conforter sa place dans celui-ci.

\section{La structure de l'espace public}

31 L'enjeu des débats est à cet égard double : d'une part, ils permettent de couvrir tout un pan du champ qui ne saurait être que marginalement servi par des manifestations comme les expositions; d'autre part, en mettant en scène avec le plus d'aisance l'interdisciplinarité qui constitue l'une des revendications premières du Centre, ils tendent à développer des problématiques transversales, des légitimités d'emprunt et des interventions latérales qui définissent la place originale du Centre dans l'espace public. Cela conduit à reconnaître que l'espace public contemporain est fortement différencié, sans que pour autant ces différences soient immédiatement hiérarchisées.

On a en effet coutume, à la suite de Jürgen Habermas, de définir l'espace public selon un modèle rationaliste hérité des Lumières, caractérisé par la liberté « de faire un usage public de sa raison dans tous les domaines ${ }^{3} »$. Ce modèle présuppose deux choses: 
d'une part, l'homogénéité de l'espace public, sinon en fait, du moins en droit, et d'autre part un partage rigoureux entre le privé et le public. On peut remarquer d'ailleurs que dans les remaniements ultérieurs de sa théorie, Habermas, parlant de rationalité communicationnelle, reprend ces deux postulats, puisque cette rationalité est à la fois le modèle idéal de toute communication réussie et aussi le fondement transcendantal caché de toute communication effective. En fait, cette représentation sert aussi de fondement à une critique des modalités de fonctionnement de l'espace public effectif, dont on va s'employer à dénoncer les distorsions: l'extension des relations marchandes, l'utilisation des médias à des fins de persuasion, le déclin des valeurs universelles, l'individualisme sous ses diverses formes viendraient compromettre jusqu'à l'existence même d'un espace public. C'est ainsi que le changement de sens qui depuis longtemps déjà a affecté le mot de publicité viendrait corroborer cette analyse : "A l'origine, la publicité garantissait le lien qu'entretenait l'usage public de la raison aussi bien avec les fondements législatifs de la domination qu'avec un contrôle critique de son exercice. Depuis, elle est au principe d'une domination qui s'exerce à travers le pouvoir de disposer d'une opinion non publique, ce qui aboutit à cette singulière équivoque : la "publicité" permet de manipuler le public, en même temps qu'elle est le moyen dont on se sert pour se justifier face à lui. Ainsi la "publicité" de manipulation prend-elle le pas sur la publicité critique ${ }^{4}$.» Dans son nouveau langage, Habermas dénoncerait la perversion de la rationalité communicationnelle en rationalité instrumentale.

Dans cette logique, il serait loisible de voir dans les débats un des ferments corrupteurs de la vie intellectuelle : par le privilège que de fait ils accordent à la parole sur l'écrit, et au-delà, à la mise en scène de soi (cf. chapitre 2) sur la réflexion rationnelle, ils ravaleraient la libre réflexion et la communication rationnelle des pensées au rang de la pure et simple confrontation d'opinions. L'élément spectaculaire, dont ils ne sont jamais complètement affranchis, représenterait au mieux l'inflexion de la notion de "publicité ». De penseur, le participant au débat deviendrait vedette médiatique. L'orientation générale du Centre vers le voir, la prégnance sur les débats du modèle télévisuel, l'acception commune du mot culture comme synonyme de loisir (c'est-à-dire ici, bien entendu, non la scholê grecque, mais le contraire du travail, c'est-à-dire ce loisir aliéné ne visant qu'à la reproduction de la force de travail, ou encore ce moment de l'affirmation de la pure différence individuelle sans pertinence publique aucune), feraient du débat le parangon de cette "idiotisation» de la vie publique, et aussi le cheval de Troie d'une dégradation de la vie intellectuelle. La concurrence avec l'Université que nous avons relevée, serait la source d'une dégénérescence de celle-ci.

34 A cette analyse, il est tentant d'opposer un autre point de vue, qui critiquant la normativité implicite de celle-ci, ne verrait dans le débat qu'un déplacement d'une logique sociale de la distinction, toujours identiquement à l'œuvre. On pourrait ainsi aisément pasticher Pierre Bourdieu en décrivant comment la participation aux débats (et particulièrement aux débats du Centre) reviendrait à développer une stratégie d'accumulation/circulation du capital culturel, dont la fonction serait de venir conforter une légitimité acquise ailleurs (et pour l'essentiel à l'Université, qui continuerait à jouer là le rôle principal) de façon à permettre que se perpétue pour un petit nombre le monopole de la détention de la culture légitime. A la fois opération de relégitimation de ce partage culturel (dont les modalités diverses d'intervention, $c f$. chapitre 3 , seraient une illustration flagrante) et création d'un nouveau niveau d'excellence de nature plus médiatique, destiné à sauver les hiérarchies existantes 
menacées par l'augmentation quantitative des universitaires et étudiants, le débat contribuerait au contraire à permettre que perdure le mythe d'un espace public d'accès égal, dans le même temps qu'on constate les limites d'institutions comme l'école quant à leur prétention à cet égard. Nouveau lieu de consécration de la culture légitime, le débat permettrait d'autant mieux de jouer ce rôle que, comme au Centre, il s'appuierait sur une utopie de la publicité, c'est-à-dire de la parole circulante et offerte à tous. Le choix stratégique d'investir les débats par les représentants des disciplines nobles (au premier chef la philosophie), à l'inverse des stratégies déployées par les disciplines scientifiques en voie de constitution, ne pourrait que venir confirmer cette analyse.

Or, ce que manquent l'une et l'autre de ces analyses, c'est la configuration effective de l'espace public. En fait, chacune postule un espace unifié, qu'il soit sous-tendu par une rationalité communicationnelle, ou qu'il soit aimanté par une hiérarchie culturelle stable. Dans un cas, on déplore sa corruption par les médias, et principalement la télévision, en soulignant les effets qu'elle induit sur la totalité de l'espace public ; dans l'autre, on décrit une continuité qui, de l'Université, irradie vers la totalité du champ. Il serait tentant d'opposer ainsi le mode de fonctionnement de la légitimité universitaire et celui de la légitimité médiatique, en considérant que le champ est principalement structuré par la lutte de l'une et de l'autre. C'est être aveugle aux emprunts incessants qui se font de l'une à l'autre, aux effets de cumul ou de résonance qui peuvent en résulter, et qui sont au moins aussi importants que les distorsions. Mieux : c'est ne pas voir que ni la légitimité universitaire, ni la légitimité médiatique n'ont de fonctionnement univoque, qu'elles sont l'une et l'autre traversées de tensions spécifiques. L'espace public, loin d'être unifié, est feuilleté, et y interfèrent les contraintes du politique, les images des identités collectives et les échos des passions individuelles. Le Centre est de ce point de vue sans nul doute un espace privilégié, dans la mesure où il rassemble et convoque la publicité la plus indifférenciée (et pas seulement sur le parvis et dans le forum, mais bien dans les débats eux-mêmes) avec les raffinements culturels les plus élaborés. Cela donne certes lieu à des effets de télescopage parfois surprenants, mais aussi contribue à proposer toute une gamme d'entrées par où se régule la consommation du Centre par son public, en fonction d'attentes de plus en plus spécifiées. Bien entendu, il y faudrait davantage de données sur les publics des débats pour pouvoir asseoir les modalités de cette différenciation, mais celle-ci se laisse deviner à la mesure même de la variété de la gamme de débats proposés.

C'est qu'ici le Centre se trouve congruent avec la structure même de l'espace public démocratique. On sait celui-ci travaillé par l'individualisme : cela ne veut pas dire que les contraintes sociales ne s'y fassent pas sentir, mais qu'elles sont elles-mêmes plurielles, et instables. La hiérarchie des légitimités par exemple, dont parle Bourdieu, n'est en aucun cas une hiérarchie stable, mais au contraire toujours sujette à des remises en cause. Les hiérarchies culturelles héritées se trouvent fortement concurrencées par d'autres formes de hiérarchies, qui mobilisent d'autres critères d'excellence : pour ne prendre qu'un exemple, la promotion d'une figure comme celle de Bernard Tapie, qui suscite tant l'ire des "cultivés », est le signe d'une contestation profonde de l'ordre des valeurs auxquelles ils adhèrent. Mais on pourrait en dire autant de tous les objets que le champ culturel englobe. Il se trouve donc que la concurrence n'est pas seulement entre individus se référant à une échelle de valeurs commune, mais entre les échelles de valeurs elles-mêmes. Dans cette concurrence, les débats du Centre jouent un rôle privilégié. En effet, d'une part, ils sont bien souvent le lieu où va se tester 
une prétention à la légitimité d'un nouvel objet (la bande dessinée, par exemple), mais aussi d'autre part, ils sont le lieu qui, adossé à la rhétorique de la création culturelle, ainsi qu'au prestige qui s'attache en France à toute institution culturelle publique, consacre la légitimité de toute prise de parole, quelle qu'elle soit. Le débat est ici la mise en scène d'une capacité performative, qui trie moins en fonction de hiérarchies établies qu'en fonction d'aptitudes pratiques à répondre, contredire, argumenter. Certes, celles-ci ne sont pas complètement sauvages, et sont aussi culturellement instituées. Mais le rapport traditionnel s'inverse, puisque on y mesure la légitimité traditionnelle à la capacité performative qu'elle confère à son détenteur, au lieu de soumettre la possibilité de la performance à la légitimité acquise.

Du moins idéalement. Car on a vu que les légitimités acquises, et notamment la légitimité universitaire, restaient très prégnantes, et mieux, que les débats tendaient à s'écarter de l'utopie fondatrice de la libre parole pour s'instituer en quasi-cursus. Cette évolution ne vient-elle pas contredire l'analyse précédente? Mais c'est que là aussi, le schéma d'un espace public livré à la pure agonistique de performances rivales relève de l'utopie, ou plutôt de la contre-utopie. On est alors dans le renversement pur et simple du modèle habermasien. Si on renonce à l'idéal d'un espace public normé par une rationalité communicationnelle, on le découvre en fait toujours traversé de tensions et d'enjeux. Mais ceux-ci ne se déploient pas arbitrairement, dans un pur rapport de forces. L'alternative n'est pas entre la communication pacifiée et la guerre de tous contre tous. Que l'enjeu principal de l'affrontement soit la question de la légitimité l'indique assez. Ce qui est en débat, dans l'espace public, ce sont précisément les critères en vertu desquels on jugera un objet comme légitime ou non, recevable ou non. Or à ce débat-là, il n'est pas de métacritères permettant de le trancher. C'est bien au point d'équilibre d'une tradition, de rapports de force et de modèles idéaux (éventuellement communicationnels) qu'il opère. Et il sous-tend souterrainement tous les débats, y compris ceux du Centre. Ceux-ci ont cependant à cet égard un privilège : situés à l'intersection des légitimités héritées et de prétentions nouvelles, du "grand public » et des " créateurs », du loisir et du savoir, ils configurent assez bien un espace microcosme de l'espace public en général. L'inflexion pédagogique doit de ce point de vue être rapportée à la réorganisation des légitimités culturelles dans l'espace public. La légitimité décroissante de l'Université s'y combine avec une diffusion croissante de savoirs, dont elle reste principalement la détentrice. Que des lieux comme le Centre apparaissent plus aptes à cette pédagogie diffuse et en même temps plus légitimes ne saurait donc surprendre. Mais cela ne veut pas dire que seules les valeurs reconnues y soient transmises, au contraire. Le recouvrement des fonctions que nous avons mis en évidence le dit bien : c'est dans le temps où le débat est lieu de production qu'il est aussi conjointement lieu de légitimation et lieu de diffusion, même si l'équilibre peut varier d'un débat à l'autre.

\section{NOTES}


2. Éthique médicale et droits de l'homme, Actes Sud, Inserm, 1988.

3. Kant, Réponse à ta question : qu'est-ce que «Les Lumières " ? Cf. aussi la deuxième section du Conflit des facultés.

4. J. Habermas, L'Espace public, Payot, 1978, p. 186. 


\section{Conclusion}

$1 \mathrm{Au}$ commencement de cette étude, nous émettions deux hypothèses : une hypothèse haute et une hypothèse basse, qui correspondaient respectivement à la possibilité que les débats du Centre soient producteurs de nouveauté dans le champ, ou au contraire qu'ils se bornent à n'avoir qu'une simple fonction de diffusion. Implicitement, le modèle était que le Centre fonctionnait dans ses débats comme une institution de médiation culturelle essentiellement, de nature analogue à la radio ou à la télévision. Très vite, il nous a fallu revenir sur ces hypothèses en constatant que la question était ainsi mal posée, et que le Centre était davantage encore à mettre en rapport avec l'Université qu'avec toute autre institution.

2 Il y a à cela plusieurs raisons. L'une des principales nous paraît être la faiblesse congénitale de l'Université française, qui n'a jamais su animer l'espace public et le champ culturel à la mesure de ce que pouvaient faire les grandes universités étrangères. Cette faiblesse est structurelle, mais elle tient aussi à l'idéologie positiviste qui présida longtemps au destin de l'Université : l'espace public y était conçu comme espace vertical de diffusion du savoir, selon un mouvement descendant, tout autre mouvement ne pouvant être que parasitage ou tentative de subversion de cet ordre. La place qu'ont toujours tenue en France, en marge de l'Université, revues, débats et autres formes d'intervention publique s'explique ainsi. A cet égard, la position du Centre nous paraît devoir être analysée dans la continuité d'une histoire. Toutefois, une conjoncture spécifique a présidé aussi à son destin en la matière. D'une part, il est venu au moment où le discours sur la culture dominant depuis le début des années soixante, celui de la démocratisation, rencontrait ses limites et se voyait concurrencé par un autre discours, celui de la création, dont le postulat implicite était la capacité expressive de chacun. L'allure ouverte du Centre devait l'installer comme utopie centrale d'un nouveau dispositif culturel, d'autant plus que ce nouveau discours, repris et amplifié par le politique, devait petit à petit devenir le discours dominant sur la culture, l'école et l'Université étant peu ou prou alors dépossédées de leur monopole en la matière, et se voyaient cantonnées dans la fabrication de savoirs techniquement et socialement utiles. Bénéficiant de ce transfert, le Centre fut ainsi sollicité de prendre en charge une fonction de pédagogie culturelle de masse qui ne trouvait pas à s'exercer ailleurs (là encore, il faudrait mettre en phase la montée en puissance du Centre et le déclin de l'utopie éducative de Vincennes, dont on retrouvera dans les débats nombre 
d'enseignants et sans doute une part du public). Intronisé ainsi temple d'une nouvelle conception de la culture, il voyait paradoxalement venir à lui un besoin qui restait encore largement déterminé par les anciens partages (culture vs profit, livre vs image, modèle culturel vs créativité individuelle). On peut dès lors comprendre qu'il ait réagi en créant des quasi-cycles universitaires, et joue ainsi le rôle d'une immense université de formation permanente, qui couvre de quasi-cursus, jusqu'au troisième cycle.

Mais à s'en tenir là on ne saisirait qu'un seul aspect du rôle et de l'évolution des débats. En effet, parallèlement, le Centre est petit à petit apparu comme le lieu de consécration des avancées culturelles reconnues. Ce rôle critique, traditionnellement plutôt dévolu à la presse et au jeu des reconnaissances entre pairs, lui est venu de l'effondrement des dernières avant-gardes esthético-politiques (l'itinéraire du groupe Tel Quel est ici significatif, qui opérera lui-même la transition vers le Centre de la fonction d'avantgarde) et de l'assomption par les grands médias d'une mission de diffusion culturelle large, incapable par nature de discrimination. Second paradoxe : le Centre se trouvait ainsi doublement légitimé, pour consacrer la validité des nouvelles valeurs culturelles, mais aussi pour promouvoir une conception beaucoup plus indifférenciée et relativiste de la hiérarchie culturelle. Les débats seront très largement les opérateurs de cette alchimie, puisque s'y donnent à voir les affrontements entre légitimités, légitimité reconnue contre légitimité prétendue.

4 Les traits que nous avons ainsi repérer dans l'évolution des débats et dans leur progressive mise en ordre se laissent ramener à une gestion plus rigoureuse de ces exigences contradictoires, qui placent le Centre au cœur d'un conflit de légitimité suscité par le mouvement même de la société démocratique moderne. Quand Michel de Certeau décrivait le Centre comme utopie "urbaine» («ce ventre pantagruélique est une ville, mais une ville qui ignore l'ancrage des demeures et des vies privées. Elle sélectionne et rassemble de l'urbain ce qui en est "public" : des bureaux, des places, des rues, des monuments»), il l'inscrivait sous le signe d'une méta-urbanité faite d'écritures et de pratiques foisonnantes. Certes, mais il faut aussi constater que le Centre se donne les moyens de hiérarchiser, toujours provisoirement, les discours qui sont tenus en son sein. Comme la ville moderne, cet espace public est un espace de classement. Comme elle, il n'obéit pas à des modalités de classement établies et arrêtées une fois pour toutes, mais sans cesse les réélabore car il organise aussi l'espace de leur mise en question. Ce monument "en dedans", destiné surtout à accueillir et faire se rencontrer ou débattre des publics, est à l'opposé de la célébration de valeurs jugées stables et consensuelles : il faut aux droits de l'homme, perçus comme le roc de notre culture commune, un monument "en dehors", visible de tous et réfractant les passions de la société : telle est l'Arche de la Défense. Le Centre, lui, les englobe, les digère, les absorbe. C'est pourquoi l'espace des débats y est congruent avec l'espace démocratique : chacun peut y venir avec son opinion, pour la confronter librement à celle d'autrui. Qu'il y ait là une bonne part d'utopie ne laisse pas de permettre en fait une circulation de la parole plus souple que partout ailleurs, où le choix est vite proposé entre la parole considérée comme légitime et celle qui n'a valeur que prise en nombre, opinion quantifiée ou sollicitée sur ce mode. Proposant un ordre des légitimités, le Centre rassure, car il trie au milieu de la profusion des discours et des pratiques. Mais en tant que cet ordre est donné comme perméable et provisoire, il sollicite sans cesse de nouveaux défis. 
5 Il était attendu qu'au terme d'une étude sur les débats, ceux-ci apparaissent comme au cœur des activités, voire de l'identité du Centre. Mais avant de s'en gausser, peut-être convient-il de se demander ce qui les rend tellement insistants, dans leur inessentialité même : s'ils n'ont pas pour eux la force des structures ou le poids des budgets, du moins permettent-ils à une parole de se frayer un chemin. Celle-ci soupèse, évalue, critique. Supposons un instant le Centre muet, et il se muerait aussitôt en un palais de la Belle au bois dormant, où la contamination du silence atteindrait toute chose. Ce déluge de paroles est ce qui assure l'urbanité du Centre, sans quoi ces places et rues seraient désertées: urbanité cahotique, parfois heurtée, mais sans cesse sollicitée, celle de l'individu mis en foule, aux antipodes de la foule solitaire. Si l'on ne devait qu'accorder ce crédit-là à la culture, il faudrait bien reconnaître que Beaubourg en est le centre. 


\section{Annexes}

Plan des départements du Centre Georges Pompidou
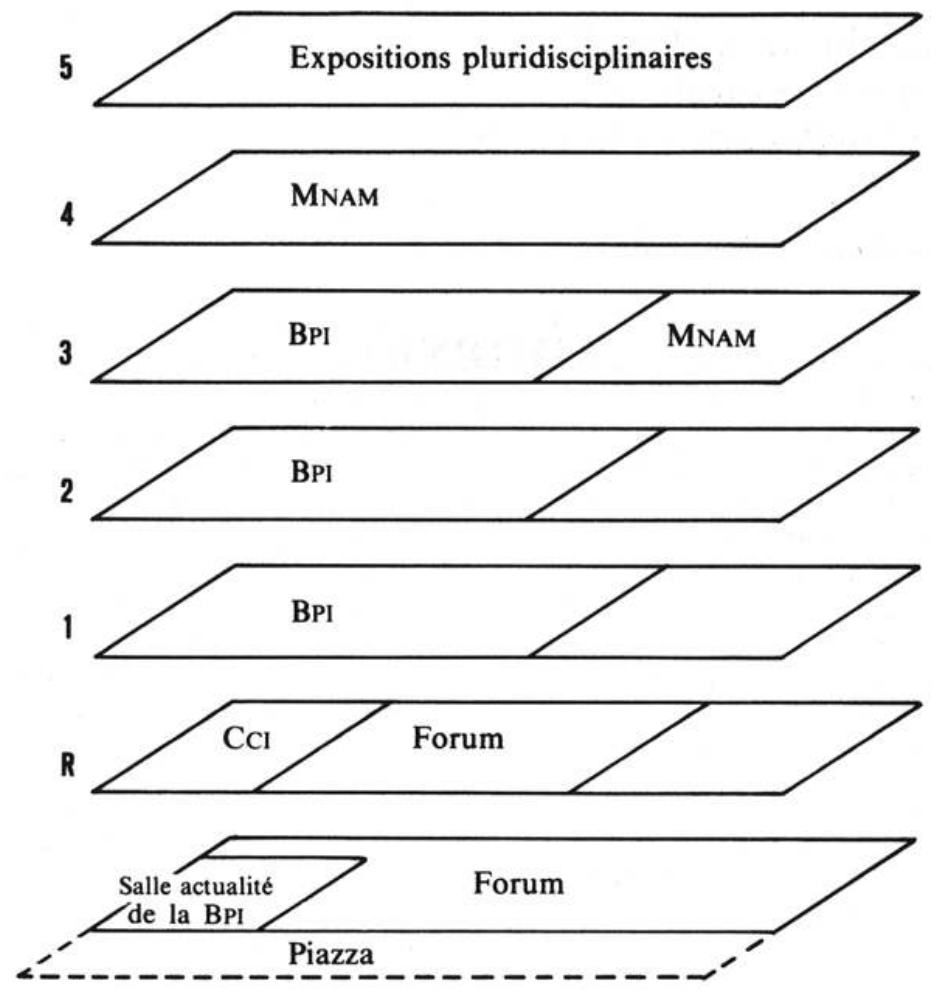

BPI: Bibliothèque publique d'information

$\mathrm{ccı}$ : Centre de création industrielle

MNAm : Musée national d'art moderne 


\section{Annexe 1. Quantification des débats selon les thématiques et les instances organisatrices}

\section{Thématiques des débats}

1 Les tableaux ci-joints ont été élaborés à partir de deux sources complémentaires : les bulletins de programmation des activités et les programmes hebdomadaires du Centre, archivés par Henri de Langle, parfois complétés par les archives spécifiques de telle ou telle instance du Centre. Les chiffres ne sont toutefois qu'indicatifs, deux sources de distorsions pouvant s'introduire: d'une part, le décalage toujours possible entre la programmation et les débats qui ont effectivement lieu; d'autre part, l'existence de débats hors-programmation, soit qu'ils interviennent trop tardivement pour pouvoir être pris en compte dans ces documents, soit qu'ils relèvent d'une initiative exceptionnelle. Mais dans les deux cas, nous avons estimé que la marge d'erreur était négligeable. Enfin, les débats de la cellule liaison-adhésion, qui ne font pas l'objet d'une annonce publique et sont recensés dans un tableau séparé.

2 Les chiffres ne doivent être lus que comme des tendances. Ils ne représentent ni des scores, ni une photographie exacte de la réalité. En effet, il peut y avoir des biais dus à l'imprécision des archives. D'autre part, l'existence des journées, cycles et colloques qui comptent plusieurs débats peuvent être à l'origine d'un effet de grand nombre.

3 Le premier trait à souligner est, toutes instances confondues, l'augmentation du nombre des débats, avec une diversification croissante des instances organisatrices et des thématiques. L'année 1983 connaît une pointe un peu artificielle, avec une vingtaine de débats «extérieurs ", consacrés aux maisons d'édition et qui forment en réalité un cycle à part. Il s'agit d'une initiative de la Maison du livre et des mots du CIRCA de Villeneuve-lès-Avignon, proposée dans le cadre du Carrefour des régions.

4 Si l'on considère les thématiques, on remarque les effets particulièrement nets de l'entrée en débats du MNAM, en 1981, qui se régularise par la suite mais continue d'exercer un quasi-monopole en ce qui concerne l'esthétique. (La division de la colonne «art visuel» en deux rubriques «esthétique» et «critique» correspond respectivement à la séparation entre les conférences du musée et son activité d'animation des expositions.) L'inversion de la proportion des débats consacrés à la littérature étrangère par rapport à la littérature française à partir de 1982, correspond au moment où l'association Dialogue entre les cultures systématise son intervention à la « Revue parlée ». La section intitulée " Problèmes de société », qui regroupe tous les débats liés à une actualité sociale (drogue, famille, etc.) et ne pouvant se rattacher légitimement à une approche disciplinaire, connaît, comme c'était prévisible, les plus grandes variations.

5 En revanche, on constate en se dirigeant vers des champs disciplinaires ou esthétiques plus précis (psychanalyse, architecture), le nombre des débats se stabilise assez vite autour d'une moyenne. Enfin, à l'évidence, la création d'instances comme l'Espace séminaire et la cellule Histoire et société sont de nature à augmenter le nombre de débats, respectivement en philosophie et en histoire.

6 Le fait le plus remarquable est qu'aucune inflexion thématique significative ne peut être relevée, autorisant à parler d'une inflexion de l'activité du Centre. 
7 Si l'on regarde les instances du Centre, on remarque que la plus grande variation joue sur la Bpi, qui est aussi la principale organisatrice de débats. La "Revue parlée » organise en moyenne une quarantaine de débats par an, le ccI, entre vingt et trente et le MNAM, une trentaine.

\section{Thématiques des débats}
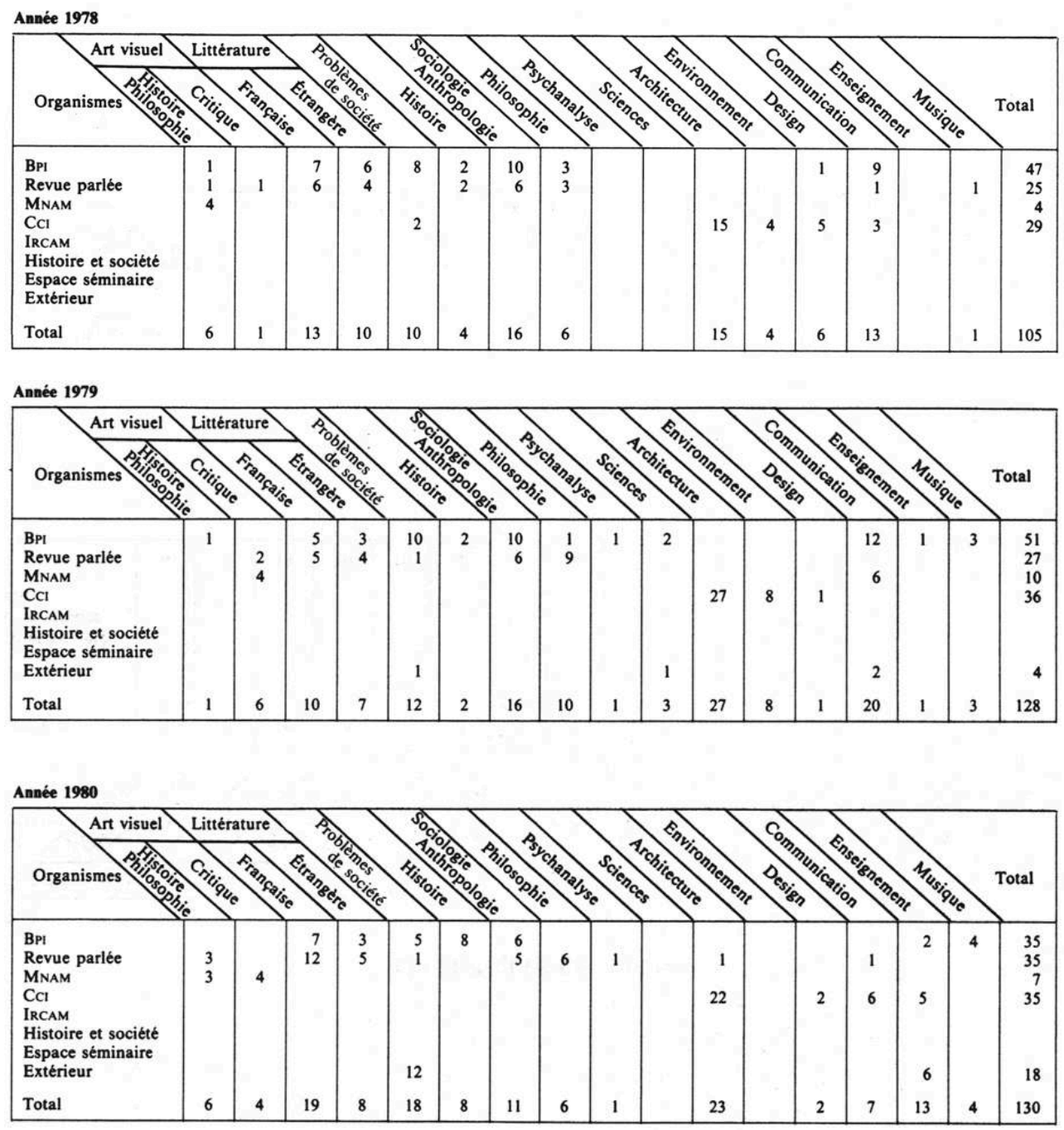
Année 1981
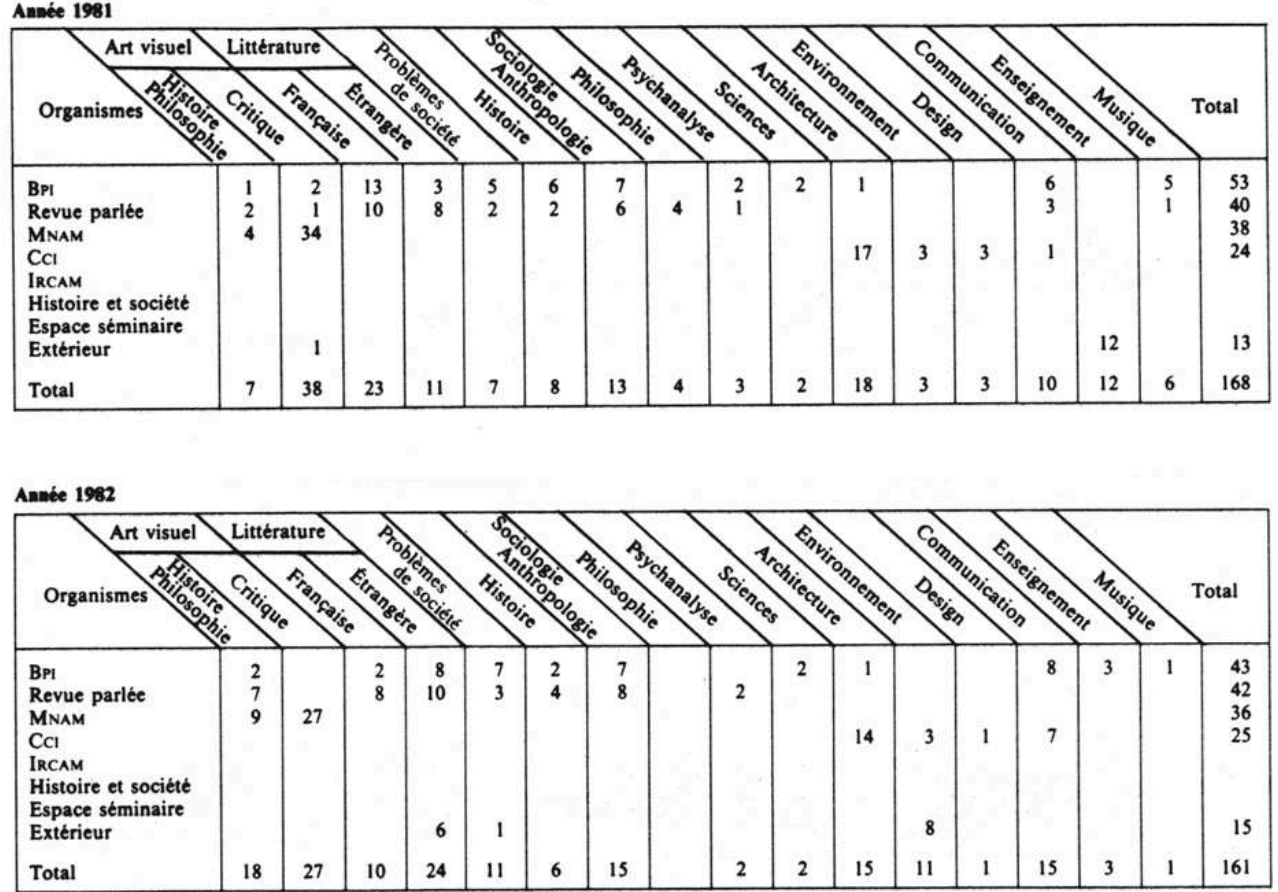

Année 1983

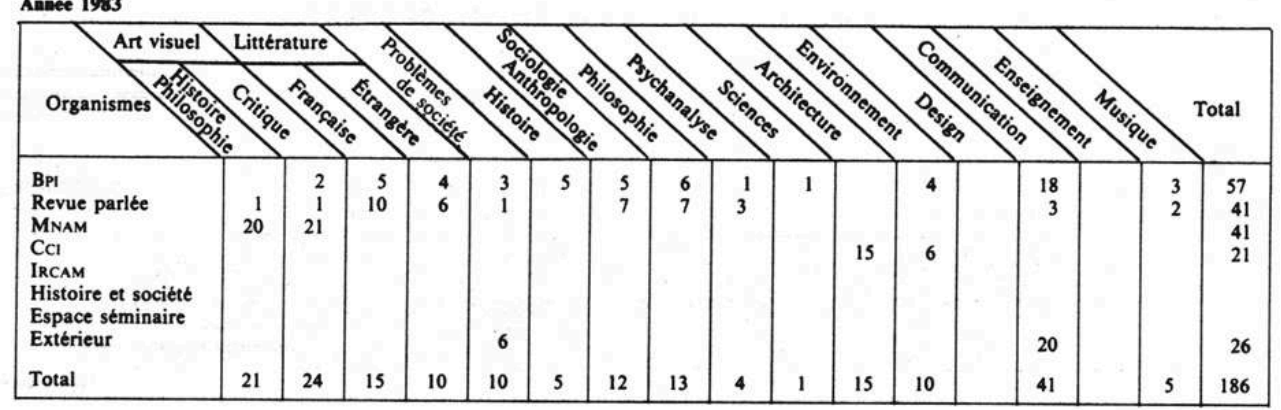

Année 1984

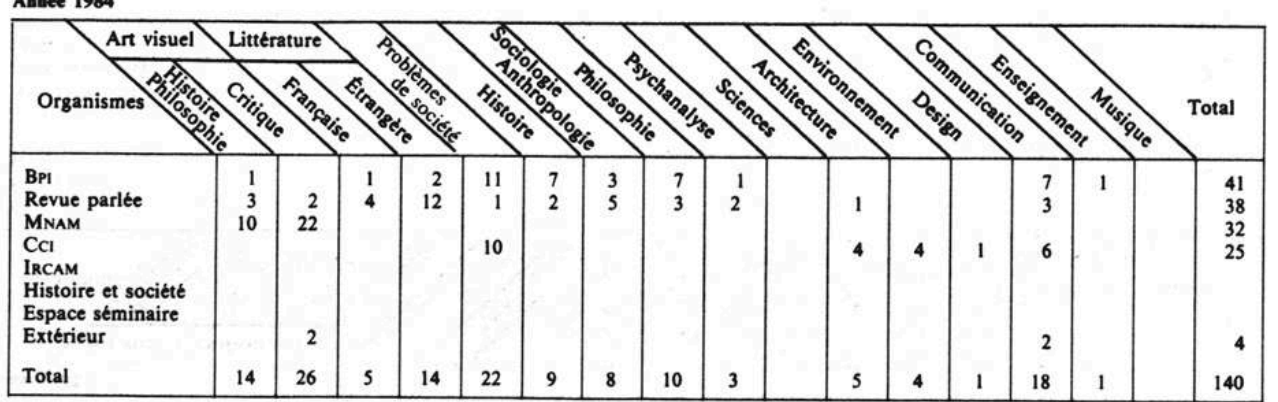



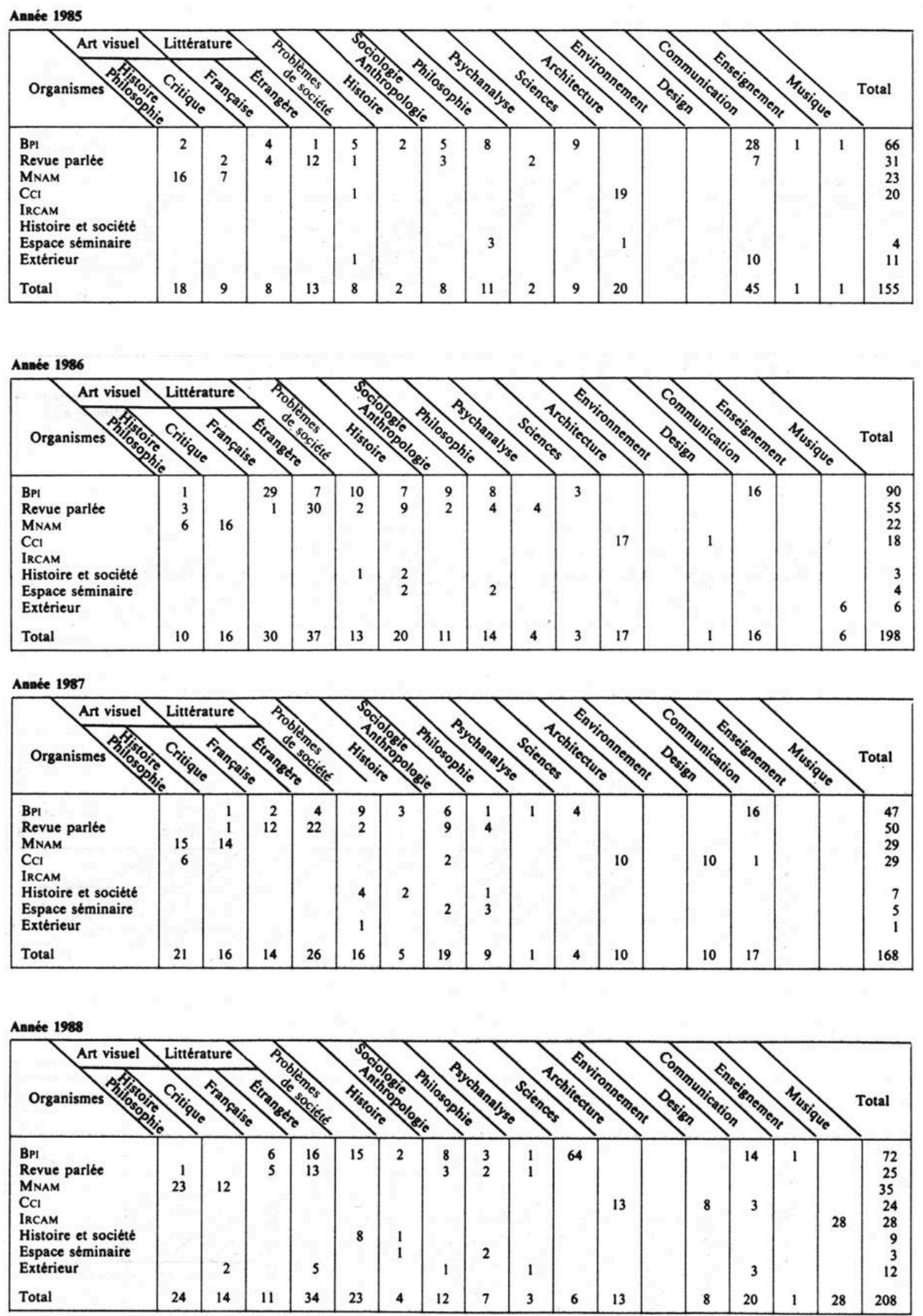

\section{Annexe 2. Cycles de la Cellule liaison/adhésion}

$1985 / 1986$

\section{Philosophie}

8 La nouvelle structure Espace séminaire dirigée par Christian Descamps, introduit au Centre Pompidou le débat philosophique. Vivre avec son temps, c'est se donner les moyens de participer aux enjeux qui sont le vif de notre époque. 
9 Ce cycle proposé aux adhérents est la prolongation de la réflexion de deux séminaires : contribution du cinéma à l'analyse historique, à partir du « cinéma de fiction et cinéma d'actualité dans l'Italie des années 1930 à 1940 ».

\section{Initiation à l'art moderne}

Cycle de quatre animations dans les collections du MNAM, assuré par un même animateur, pour un groupe limité de participants, selon un parcours permettant d'aborder l'ensemble des mouvements artistiques du XX ${ }^{e}$ siècle.

\section{Musique à Vienne}

11 Une série de vingt-deux concerts marque la participation de L'IRCAM à l'exposition Vienne 1880-1938. Un cycle de conférences illustrées de documents visuels et de citations musicales permettra de restituer la vie musicale à Vienne et son climat de polyvalence artistique.

\section{Le Centre de création industrielle}

12 Les expositions du CCI visent à rendre compte des relations entre les individus, les espaces, les objets et les signes. Trois conférences illustrées fourniront introduction et repères pour en aborder les aspects les plus constitués : architecture, design, et les plus récents: technologies nouvelles et innovation sociale, en concomitance avec trois expositions : Achille Castiglioni, du ready-made au design, Plecnik et Lieux de travail.

\section{$1986 / 1987$}

Japon

13 En liaison avec l'exposition Le Japon des avant-gardes une série de dialogues plutôt que de conférences, pour tenter d'approcher quelques axes de la pensée japonaise - repères sur l'anthropologie, la linguistique, l'esthétique... et introduire aux données du Japon contemporain dans le domaine des arts plastiques et de la musique, où s'expriment les contradictions entre tradition et modernité, et dans celui du cinéma, qui les illustre.

\section{L'exposition culturelle : un nouveau média}

Des monographies aux expositions documentaires, aux fresques pluridisciplinaires : un élargissement des thèmes ; de l'œuvre, au document, à la mise en scène : une évolution de l'objet; du conservateur à l'auteur: un changement de conception. Comment le public, consommateur d'expositions, en décode-t-il le sens? Par la confrontation d'expériences et de réflexions réunissant concepteurs d'expositions, sociologues et adhérents, ce cycle constitue une tentative pour susciter un regard critique.

\section{Musique/peinture : convergences}

15 Stravinsky/Picasso, Schoenberg/Kandinsky, Webem/Mondrian, Boulez/Klee... Le rapprochement de créateurs de disciplines différentes, entre lesquels apparaissent des convergences de sensibilité, de démarches, de style mettra en lumière certains parallèles dans le mouvement de la création. Cycle de conférences illustrées de 
projections et extraits d'œuvres jouées par les solistes de l'ensemble InterContemporain.

\section{Initiation à Part moderne}

16 Cycle de quatre animations dans les collections du MNAM, assuré par un même animateur, pour un groupe limité de participants, selon un parcours permettant d'aborder l'ensemble des mouvements artistiques du $\mathrm{XX}^{\mathrm{e}}$ siècle. Deux sessions, en janvier ou en juin, selon un large choix de jours et d'horaires, à raison d'une séance d'une heure et demie par semaine et un cycle concentré sur deux ou trois jours consécutifs, organisé à la demande des adhérents de province.

\section{8/1989}

\section{Initiation à Part moderne}

17 Parcours des collections du MNAM, en quatre séances, permettant d'aborder l'ensemble des mouvements artistiques du $\mathrm{XX}^{\mathrm{e}}$ siècle, par petits groupes, sous la conduite d'un animateur.

Plusieurs cycles de janvier à juin 1989 seront proposés, selon un large choix de jours et d'horaires.

\section{Artiste et société}

19 Percevoir les œuvres d'art ne consiste pas uniquement à voir des objets ; nous voyons aussi l'artiste derrière l'objet.

Or la figure de l'artiste n'a pas toujours été la même. Nous connaissons tous la figure romantique du créateur maudit «à la Van Gogh»; il y a aussi les artistes officiels, comme David; et d'autres figures encore: l'artiste-artisan, l'artiste-entrepreneur, l'artiste primitif anonyme. Dans ce cycle de cinq conférences, Yves Michaud examinera, en rapport avec la perception de l'art contemporain, les métamorphoses de l'artiste multiforme.

\section{Promenades architecturales}

21 Une approche directe de l'architecture, au travers de parcours, en petits groupes, sous la conduite d'un architecte, évoquant des moments, des styles, des typologies de l'architecture parisienne: ateliers d'artistes à Montmartre/Montparnasse des années trente, architectes contemporains/villes nouvelles...

\section{L'exposition explorée}

Par quels moyens spécifiques l'exposition produit-elle sens et émotion?

Des ateliers parcours au travers de diverses expositions dans et hors du Centre permettront d'analyser les éléments de mise en discours/mise en espace d'objets, complétés par des rencontres avec les professionnels qui œuvrent dans les coulisses des expositions : architectes, graphistes, éclairagistes... 


\section{$1989 / 1990$}

\section{Art urbain promenades architecturales}

$\mathrm{XX}^{\mathrm{e}}$ siècle, permettra d'étudier les œuvres architecturales (style, composantes, histoire...), le contexte social de commande, de planification, d'évolution urbanistique : l'axe historique Louvre-Défense, Guimard et l'Art nouveau, le XVI ${ }^{e}$ dans l'entre-deuxguerres, architecture et lieux de travail, ville et décors éphémères, les murs peints, etc.

\section{Musique passage au $\mathrm{XX}$ siècle}

Approche vivante de la musique de notre temps, cette initiation à l'évolution du langage musical est destinée à tous ceux qui s'interrogent sur les bouleversements survenus depuis le début de ce siècle. Deux conférences de Cécile Gilly, illustrées de nombreux exemples musicaux, de Mozart à Wagner, Schoenberg, Stravinsky, Messiaen, Boulez, Berio, Cage, Stockhausen... Avec la participation des solistes de l'Ensemble intercontemporain.

\section{Six leçons de design}

- modernisme à l'heure industrielle : 1850-1990 ;

- Art nouveau, Sécession, art déco ;

- Le filon constructiviste et rationaliste ;

- crise économique de 1929 et le lancement du styling ;

- L'après-guerre et les formes libres ;

- Crises et confessions : les mouvements contemporains.

Conférences par François Burkhardt et Raymond Guidot, illustrées de projections.

\section{De Matisse à nos jours ; initiation à l'art moderne}

Parcours des collections du MNAM, en quatre séances, permettant d'aborder l'ensemble des mouvements artistiques du XXe siècle : le fauvisme, le cubisme, les abstractions, le surréalisme, le Pop'Art... jusqu'aux courants contemporains.

Les débats de la Cellule liaison/adhésion

\begin{tabular}{|c|c|c|c|c|c|c|c|c|c|c|c|c|}
\hline \multirow[b]{2}{*}{ Années } & \multicolumn{3}{|c|}{ Rencontres } & \multicolumn{4}{|c|}{ Tables rondes/débats } & \multicolumn{3}{|c|}{ Cycles de formation } & \multirow[b]{2}{*}{ Littérature } & \multirow[b]{2}{*}{ Total } \\
\hline & $\begin{array}{c}\text { Art } \\
\text { visuel }\end{array}$ & Architecture & Musique & $\begin{array}{c}\text { Art } \\
\text { visuel }\end{array}$ & Architecture & Musique & $\begin{array}{c}\text { Philosophie } \\
\text { Sciences } \\
\text { humaines }\end{array}$ & $\begin{array}{c}\text { Art } \\
\text { visuel }\end{array}$ & Architecture & Musique & & \\
\hline $\begin{array}{l}1978 \\
1979\end{array}$ & 2 & 1 & 1 & 1 & & & & 18 & & 4 & & 27 \\
\hline 1980 & 8 & & & 6 & & & 1 & & & 6 & & $22^{3}$ \\
\hline & 4 & & 1 & 10 & 7 & 5 & 2 & 4 & & 2 & & 35 \\
\hline 1982 & 6 & & 1 & 1 & & & 3 & 8 & & 6 & & 25 \\
\hline $\begin{array}{l}1983 \\
1984\end{array}$ & 1 & & & & 1 & & & & 3 & 4 & & 9 \\
\hline & 1 & & & 2 & & & 1 & & 8 & 3 & & 12 \\
\hline 1986 & & & & & 1 & & 1 & 8 & & ${ }_{2}^{8}$ & & \\
\hline 1987 & & 1 & & & & & & 15 & 4 & 2 & 5 & 27 \\
\hline 1988 & $1 "$ & & & & & & 9 & 4 & & & & 14 \\
\hline $\begin{array}{l}1989 \\
1990\end{array}$ & & & & & & & 8 & $\begin{array}{l}16 \\
10\end{array}$ & 6 & & $\begin{array}{l}6 \\
1\end{array}$ & $\begin{array}{l}36 \\
11\end{array}$ \\
\hline Total & 25 & 2 & 3 & 20 & 10 & 5 & 26 & 86 & 21 & 37 & 12 & 247 \\
\hline
\end{tabular}




\section{Annexe 3. Une année de débats au Centre Georges Pompidou : 1986}

\begin{tabular}{|c|c|}
\hline $\begin{array}{l}\text { Cellule } \\
\text { liaison/ } \\
\text { adhésion }\end{array}$ & $\begin{array}{l}8 \text { janvier } \\
\text { Cinéma d'actualité et cinéma de fiction dans l'Italie des années 1930-1940 }\end{array}$ \\
\hline MNAM & $\begin{array}{l}9 \text { janvier } \\
\text { L'art augural de Valerio Adami par Paolo Fabbri }\end{array}$ \\
\hline BPI & $\begin{array}{l}\text { Marco Polo, une expérience d'écriture plurielle avec B. Tournois, J. Lacarrière, C. } \\
\text { Matisse, F. Delay, B. Visage, B. Rastoin ; animation : B. de Fréminville. }\end{array}$ \\
\hline MNAM & $\begin{array}{l}16 \text { janvier } \\
\text { Les noms propres d'Adami } \\
\text { Conférence de René Major. }\end{array}$ \\
\hline BPI & $\begin{array}{l}\text { Autour du livre Contre l'art d'élite de William Morris, avec J. Gattegno, J.-P. Richard, F. } \\
\text { Burkhardt. }\end{array}$ \\
\hline $\begin{array}{l}\text { Revue } \\
\text { parlée } \\
\text { BPI }\end{array}$ & $\begin{array}{l}22 \text { janvier } \\
\text { Trieste, ville de l'écriture } \\
\text { Cabinet de lecture : «L'individu », avec É. Balibar, J. Rogozinski. }\end{array}$ \\
\hline $\begin{array}{l}\text { BPI/Le Matin } \\
\text { de Paris }\end{array}$ & $\begin{array}{l}23 \text { janvier } \\
\text { Histoire de l'intelligentsia } \\
\text { Débat avec P. Assouline, L. Ferry, A. Renaut, M. Gallo, H. Hamon, P. Rotman, J. } \\
\text { Negroni, P. Nora ; animation : A. Spire. }\end{array}$ \\
\hline MNAM & $\begin{array}{l}\text { Collections et muséographies } \\
\text { Table ronde avec J. Leymarie, F. Meyer, D. Sylvester, E. de Wild, H. Szeeman, } \\
\text { animation: C. Millet. }\end{array}$ \\
\hline $\begin{array}{l}\text { Revue } \\
\text { parlée }\end{array}$ & Trieste, ville de l'écriture \\
\hline $\mathrm{CCI} / \mathrm{IFA}^{1}$ & $\begin{array}{l}24 \text { janvier } \\
\text { Lumières. Rencontres « Voir et Mouvoir » avec A. Diot, Y. Kersalé, G. Lafosse, T. } \\
\text { Benoit }\end{array}$ \\
\hline \multirow[t]{2}{*}{$\begin{array}{l}\text { Revue } \\
\text { parlée }\end{array}$} & $\begin{array}{l}27 \text { janvier } \\
\text { Trouver Trieste : « Psychiatrie et lois, le droit du malade» } \\
\text { avec F. Ongaro-Basaglia. }\end{array}$ \\
\hline & $\begin{array}{l}29 \text { janvier } \\
\text { Droit d'asile aujourd'hui et demain } \\
\text { Conférence de presse : «La situation des réfugiés en France ». Vente de livres. }\end{array}$ \\
\hline $\begin{array}{l}\text { Revue } \\
\text { parlée }\end{array}$ & $\begin{array}{l}\text { Trouver Trieste : « Voix Slovènes » } \\
\text { avec E. Bavçar. }\end{array}$ \\
\hline
\end{tabular}




\begin{tabular}{|c|c|}
\hline BPI & $\begin{array}{l}30 \text { janvier } \\
\text { L'Inde : actualité politique et sociale } \\
\text { avec J.-A. Bernard, René Major...; animation : Henri Stem. }\end{array}$ \\
\hline MNAM & $\begin{array}{l}\text { L'art en situation } \\
\text { Conférence de Philippe Sollers. }\end{array}$ \\
\hline $\begin{array}{l}\text { Revue } \\
\text { parlée }\end{array}$ & Trouver Trieste : « Voix Slovènes ». \\
\hline $\begin{array}{l}\text { Revue } \\
\text { parlée }\end{array}$ & $\begin{array}{l}31 \text { janvier } \\
\text { Trouver Trieste : « Le néo-classicisme » } \\
\text { avec P.-L. Nicolin, D. Portofirios, D. Portoghesi, G. Vallet ; présentation : L. Semerani. }\end{array}$ \\
\hline $\begin{array}{l}\text { Cellule } \\
\text { Liaison/ } \\
\text { Adhésion }\end{array}$ & $\begin{array}{l}\text { Introduction à la création industrielle } \\
\text { avec F. Burkhardt, F. Jollant et R. Guidot. }\end{array}$ \\
\hline $\begin{array}{l}\text { Revue } \\
\text { parlée }\end{array}$ & $\begin{array}{l}3 \text { février } \\
\text { Trouver Trieste : « Rilke et Trieste» } \\
\text { conception : J. Clausel. }\end{array}$ \\
\hline BPI & $\begin{array}{l}6 \text { février } \\
\text { La littérature yougoslave aujourd'hui }\end{array}$ \\
\hline $\begin{array}{l}\text { Revue } \\
\text { parlée }\end{array}$ & $\begin{array}{l}\text { Trouver Trieste : « Le pari scientifique de Trieste » } \\
\text { avec M. Aymard, A. Borsellino, P. Budinic, M. Lévy, E. Panizon, N. Tamburini, G. Toti ; } \\
\text { présentation : G.-P. de Ferra. }\end{array}$ \\
\hline BPI & $\begin{array}{l}10 \text { février } \\
\text { Journées d'étude : la critique des livres pour la jeunesse } \\
\text { Animation : J. Pinguilly. }\end{array}$ \\
\hline BPI & $\begin{array}{l}19 \text { février } \\
\text { Cabinet de lecture } \\
\text { Débat autour de "Sartre: critique de la raison dialectique "; animation: J.-M. } \\
\text { Vincent. }\end{array}$ \\
\hline $\begin{array}{l}\text { Revue } \\
\text { parlée }\end{array}$ & $\begin{array}{l}20 \text { février } \\
\text { Rencontres franco-germaniques } \\
\text { 1. Entretien avec G. Grass et A. Robbe-Grillet } \\
\text { 2. Question du sens et rationalité } \\
\text { 3. Crise des idéologies et pouvoir de la raison }\end{array}$ \\
\hline MNAM & $\begin{array}{l}\text { La découverte des dissonances } \\
\text { Conférence par le professeur W. Hofmann dans le cadre de l'exposition Vienne } \\
\text { 1880-1938. }\end{array}$ \\
\hline BPI & Les risques technologiques Conversation-rencontre. \\
\hline
\end{tabular}




\begin{tabular}{|c|c|}
\hline $\begin{array}{l}\text { Revue } \\
\text { parlée }\end{array}$ & $\begin{array}{l}22 \text { février } \\
\text { Vienne 1880-1938 } \\
\text { 1. Expérience freudienne/Sciences bio-neuro-médicales } \\
\text { 2. Expérience freudienne/Arts et lettres } \\
\text { Débats, tables rondes. }\end{array}$ \\
\hline $\begin{array}{l}\text { Revue } \\
\text { parlée }\end{array}$ & $\begin{array}{l}23 \text { février } \\
\text { Vienne 1880-1938 } \\
\text { 1. Expérience freudienne/La psychanalyse } \\
\text { 2. Expérience freudienne/Clinique et théorie } \\
\text { Débats, tables rondes. }\end{array}$ \\
\hline $\begin{array}{l}\text { Revue } \\
\text { parlée }\end{array}$ & $\begin{array}{l}26 \text { février } \\
\text { William Johnston } \\
\text { Présentation de son dernier ouvrage L'Esprit viennois. } \\
\text { Présentation de R. Jaccard, en présence de l'auteur; avec la participation de J. Le } \\
\text { Rider; dans le cadre de l'exposition Vienne 1880-1938. }\end{array}$ \\
\hline $\begin{array}{l}\text { Revue } \\
\text { parlée }\end{array}$ & $\begin{array}{l}27 \text { février } \\
\text { Rencontres franco-germaniques } \\
\text { 1. Littérature : formes nouvelles d'écriture } \\
\text { 2. Cinéma : écriture du moi, écriture du nous }\end{array}$ \\
\hline MNAM & $\begin{array}{l}\text { Sur la théorie de l'art, celle de Riegl en particulier } \\
\text { Conférence par le professeur H. Zemer, } \\
\text { dans le cadre de l'exposition Vienne 1880-1938. }\end{array}$ \\
\hline BPI & $\begin{array}{l}\text { « Tintín » dans tous ses ébats } \\
\text { Conversation-rencontre. }\end{array}$ \\
\hline BPI & $\begin{array}{l}28 \text { février } \\
\text { Journées d'étude } \\
\text { A propos du livre de Charles Morazé : L'Eil d'Horus : origine sacrée des sciences modernes. }\end{array}$ \\
\hline $\begin{array}{l}\text { Revue } \\
\text { parlée }\end{array}$ & $\begin{array}{l}6 \text { mars } \\
\text { Rencontres franco-germaniques } \\
\text { 1. Mise en scène, mise en question } \\
\text { 2. De l'art à la scène théâtrale }\end{array}$ \\
\hline BPI & $\begin{array}{l}\text { Les rencontres de la traductière } \\
\text { Ateliers de traduction et de lecture ; animation : Jacques Rancourt. } \\
\text { Conversation-rencontre. }\end{array}$ \\
\hline MNAM & $\begin{array}{l}\text { Picabia, Van Doesburg, Morellet, ou la géométrie dévoyée } \\
\text { Conférence de Bernard Blistène. }\end{array}$ \\
\hline $\begin{array}{l}\text { Revue } \\
\text { parlée }\end{array}$ & $\begin{array}{l}7 \text { mars } \\
\text { Sri Aurobindo. « A Great Soul of India » } \\
\text { présenté par Yogacharya Sri Dinesh, son disciple. }\end{array}$ \\
\hline
\end{tabular}




\begin{tabular}{|c|c|}
\hline $\begin{array}{l}\text { Revue } \\
\text { parlée }\end{array}$ & $\begin{array}{l}13 \text { mars } \\
\text { Rencontres franco-germaniques } \\
\text { 1. Rôle nouveau des exigences éthiques dans la vie politique } \\
\text { 2. Luttes sociales et consensus }\end{array}$ \\
\hline BPI & $\begin{array}{l}\text { L'Algérie : actualité politique et sociale } \\
\text { Conversation-rencontre. }\end{array}$ \\
\hline BPI & $\begin{array}{l}\text { Vienne 1918-1938 } \\
\text { avec l'ex-chancelier Bruno Kreisky. }\end{array}$ \\
\hline BPI & $\begin{array}{l}14 \text { mars } \\
\text { A propos de Bruno Kreisky } \\
\text { Soirée littéraire avec l'ex-chancelier Bruno Kreisky. }\end{array}$ \\
\hline BPI & $\begin{array}{l}15 \text { mars } \\
\text { La nuit venue }: 1915^{(*)} \\
\text { Aphorismes de Karl Kraus traduits et lus par Roger Lewinter. }\end{array}$ \\
\hline BPI & $\begin{array}{l}16 \text { mars } \\
\text { La nuit venue : psychanalyse }\left(^{(*)}\right. \\
\text { Aphorismes de Karl Kraus traduits et lus par Roger Lewinter. }\end{array}$ \\
\hline & $\begin{array}{l}17 \text { mars } \\
\text { La critique de livres pour la jeunesse } \\
\text { Journée d'étude organisée avec le concours de la revue Trousse livres; animation : Y. } \\
\text { Pinguilly. }\end{array}$ \\
\hline MNAM & $\begin{array}{l}\text { Wittgenstein et Goethe }{ }^{(*)} \\
\text { Conférence musée de Jacques Bouveresse. }\end{array}$ \\
\hline BPI & Paris-Vienne, Hermann Bahr et la France ${ }^{(*)}$ \\
\hline CCI & $\begin{array}{l}19 \text { mars } \\
\text { Une architecture sans avant-garde }{ }^{(*)} \text { Débat avec C. E. Schorske, D. Worbs, J. Spalt. }\end{array}$ \\
\hline MNAM & Rencontre avec François Morellet $\left.{ }^{*}\right)$ \\
\hline CCI & $\begin{array}{l}20 \text { mars } \\
\text { Habiter à Vienne } \\
\text { Conférence avec E. Sekler, H. Czech, R. Kohoutek, G. Pirhofer. }\end{array}$ \\
\hline BPI & $\begin{array}{l}\text { L'intelligence artificielle } \\
\text { Conversation-rencontre; animation : Pierre Vandegynste. }\end{array}$ \\
\hline BPI & Le mythe viennois dans la littérature française $\mathrm{du} \mathrm{XX}^{\mathrm{e}}$ siècle \\
\hline BPI & $\begin{array}{l}21 \text { mars } \\
\text { Colloque : Karl Kraus }\left(^{*}\right) \\
\text { Dir. scientifique G. Stieg et S.-P. Scheichl. }\end{array}$ \\
\hline
\end{tabular}




\begin{tabular}{|c|c|}
\hline BPI & $\begin{array}{l}22 \text { mars } \\
\left.\text { Colloque : Karl Kraus }{ }^{*}\right) \\
\text { Dir. scientifique G. Stieg et S.-P. Scheichl. }\end{array}$ \\
\hline BPI & $\begin{array}{l}23 \text { mars } \\
\left.\text { Colloque : Karl Kraus }{ }^{*}\right) \\
\text { Dir. scientifique G. Stieg et S.-P. Scheichl. }\end{array}$ \\
\hline MNAM & $\begin{array}{l}24 \text { mars } \\
\text { Vienne au tournant du siècle : objet historique ou d'actualité }\left(^{*}\right) \\
\text { Table ronde avec W. Hoffmann, C.E. Schorske, H. Hollein. Animation : M. Pollak et N. } \\
\text { Heinich. }\end{array}$ \\
\hline CCI & $\begin{array}{l}26 \text { mars } \\
\text { Architecture et construction }\end{array}$ \\
\hline $\begin{array}{l}\text { Revue } \\
\text { parlée }\end{array}$ & $\begin{array}{l}\text { La création contemporaine anglaise } \\
\text { En liaison avec la revue Autrement et le British Council. }\end{array}$ \\
\hline $\begin{array}{l}\text { Revue } \\
\text { parlée }\end{array}$ & $\begin{array}{l}27 \text { mars } \\
\text { La création contemporaine anglaise } \\
\text { En liaison avec la revue Autrement et le British Council. }\end{array}$ \\
\hline BPI & $\begin{array}{l}\text { La littérature autrichienne d'après-guerre }\left(^{*}\right) \\
\text { avec H.C. Artmann, G.A. Goldschmidt, D. Hornig, A. Kolleritsch, J.-L. de Rambures. } \\
\text { Animation : Jean-Louis Poitevin. }\end{array}$ \\
\hline $\begin{array}{l}\text { Revue } \\
\text { parlée }\end{array}$ & $\begin{array}{l}4 \text { avril } \\
\text { Autriche, itinéraire politique et social ' } \\
\text { F. Kreissler : A la croisée des chemins : rêves et réalités dans l'histoire autrichienne, } \\
\text { 1867-1939 »; J.-P. Bled : Le compromis austro-hongrois, } 1867 \text { " C. Weill : La question } \\
\text { des nationalités, 1867-1918 »; J. Droz : Schönerer et l'antisémitisme autrichien ». }\end{array}$ \\
\hline $\begin{array}{l}\text { Revue } \\
\text { parlée }\end{array}$ & $\begin{array}{l}5 \text { avril } \\
\text { Autriche, itinéraire politique et social (*)' } \\
\text { H. Steiner : La naissance du mouvement ouvrier en Autriche-Hongrie ; } \\
\text { B. Michel : La société austro-hongroise est-elle en décadence avant } 1914 \text { ? ; } \\
\text { S. von Reden : L'Autriche-Hongrie, un état multiconfessionnel »; } \\
\text { E. Bielka : Les manipulations à l'occasion des trois plébiscites pour l'Anschluss, } \\
\text { 1919-1938 "; } \\
\text { F. Pichler : Continuité et discontinuité dans la police viennoise »; } \\
\text { I. Ackerl : L'État autoritaire, 1933-1938 ». }\end{array}$ \\
\hline
\end{tabular}




\begin{tabular}{|c|c|}
\hline $\begin{array}{l}\text { Revue } \\
\text { parlée }\end{array}$ & $\begin{array}{l}6 \text { avril } \\
\text { Autriche, itinéraire politique et social }(*) \\
\text { M. Cullin : Les années trente : le déclin d'une République "; } \\
\text { U. Weber : Stratégies contre le chômage entre les deux guerres mondiales "; } \\
\text { P. Pasteur : Die Unzufriedene : un journal féminin pas comme les autres "; } \\
\text { I. Etzesdorfer : Février } 1934 \text { - Dissolution des structures démocratiques en } \\
\text { Autriche »; } \\
\text { R. Thieberger: Le Burgtheater de ma jeunesse, 1924-1934. Souvenirs nostalgiques et } \\
\text { critiques "; } \\
\text { C. Itzigson: Sciences et scientifiques autrichiens au tournant du siècle ». }\end{array}$ \\
\hline $\begin{array}{l}\text { Revue } \\
\text { parlée }\end{array}$ & $\begin{array}{l}7 \text { avril } \\
\text { Les projets éducatifs et l'utopie de l'homme nouveau }\left(^{*}\right) \\
\text { 1. Histoire d'une exposition, avec H. Maimann. Animation : Ch. Carrier } \\
\text { 2. Historique et politique de la radio-diffusion en Autriche, 1924-1938, avec R. } \\
\text { Wischenbart. Animation : G. Zeissel. } \\
\text { 3. La social-démocratie : projets éducatifs, projets culturels, avec F. Kreissler, G. Stieg, } \\
\text { J. Rovan, I. Etzersdorfer, H. Altenhuber, A. Psigan, W. Filia, J. Wezidenholzer, M. } \\
\text { Cullin. }\end{array}$ \\
\hline $\mathrm{CCI}$ & $\begin{array}{l}9 \text { avril } \\
\text { Techniques et expression de commande : un débat contradictoire } \\
\text { avec P. Chemetov, D. Perrault, F. Soler. Animation : A. Pelissier. Architecture et } \\
\text { construction. }\end{array}$ \\
\hline $\begin{array}{l}\text { Revue } \\
\text { parlée }\end{array}$ & $\begin{array}{l}10 \text { avril } \\
\text { Autriche, itinéraire culturel }(*) \\
\text { R. Bauer, P. Kampits, G. Kars, W. Hofer, G. Castellan, G. Cerha. }\end{array}$ \\
\hline BPI & $\begin{array}{l}\text { Les stratégies européennes de la télédistribution } \\
\text { Autour de la revue Nouvelles Communications. } \\
\text { Conversation-rencontre. Animation: Roger Caracache. }\end{array}$ \\
\hline BPI & $\begin{array}{l}\text { Les Juifs et Vienne }\left(^{*}\right) \\
\text { Avec Michaël Löwy. }\end{array}$ \\
\hline $\begin{array}{l}\text { Revue } \\
\text { parlée }\end{array}$ & $\begin{array}{l}11 \text { avril Autriche, itinéraire culturel }(*) \\
\text { G. Ravy, A. Soulez, D. Ronte, S. Loewe, G. Stieg, P. Por, H.-P. Wittmann. Colloque. } \\
\text { Direction scientifique F. Kreissler. }\end{array}$ \\
\hline $\begin{array}{l}\text { Revue } \\
\text { parlée }\end{array}$ & $\begin{array}{l}12 \text { avril } \\
\text { Autriche, itinéraire culturel }(*) \\
\text { R. Thalmann, P. Stockinger, J. Sebestik, Ch. Chauviré, D. Kaufmann, G. Badia, U. } \\
\text { Birbaumer, J. Doll, D. Homig, A. Pfersmann. Colloque. Dir. scientifique F. Kreissler. }\end{array}$ \\
\hline $\mathrm{CCI}$ & $\begin{array}{l}14 \text { avril } \\
\text { Vienne, espace vital et formes architecturales }\left(^{*}\right) \\
\text { Table ronde avec D. Wieczorek, K. Novy, K. Mang, G. Kähler. }\end{array}$ \\
\hline MNAM & $\begin{array}{l}\text { Que reste-t-il du constructivisme dans l'art contemporain? } \\
\text { Conférence de Claude Gintz. }\end{array}$ \\
\hline
\end{tabular}




\begin{tabular}{|c|c|}
\hline CCI & $\begin{array}{l}16 \text { avril } \\
\text { Éducation et culture : à la recherche d'une réponse technologique avec A. } \\
\text { Fainsilber, J.-F. Bonne, J.-C. Fougeron et Architecture Studio. } \\
\text { Architecture et construction. }\end{array}$ \\
\hline BPI & $\begin{array}{l}\text { L'amour } \\
\text { Autour de la revue Le Genre humain. Conversation-rencontre avec M. Benabou, Ch. } \\
\text { David, L. Flem, F. Ouere, Y. Hersant, B. Roy. Animation : N. Fresco. }\end{array}$ \\
\hline $\mathrm{CCI}$ & $\begin{array}{l}17 \text { avril } \\
\text { Internationalisme, multinationalisme et identité }(*) \\
\text { Table ronde avec A. Kubova, P. Haiko, F. Achleitner. }\end{array}$ \\
\hline BPI & $\begin{array}{l}\text { Puissance des masses et genèse des despotismes }\left({ }^{*}\right) \\
\text { Animation : René Major. }\end{array}$ \\
\hline BPI & $\begin{array}{l}18 \text { avril } \\
\text { Colloque national « L'enfant et la poésie " } \\
\text { Poésie et chansons. }\end{array}$ \\
\hline BPI & $\begin{array}{l}19 \text { avril } \\
\text { Colloque national «L'enfant et la poésie » } \\
\text { La poésie dans l'éducation d'aujourd'hui } \\
\text { Situation de la poésie contemporaine } \\
\text { La poésie contemporaine et l'enfant } \\
\text { Comptes rendus d'expériences } \\
\text { La poésie et ses publics. }\end{array}$ \\
\hline BPI & $\begin{array}{l}20 \text { avril } \\
\text { Colloque national « L'enfant et la poésie " } \\
\text { La création et l'enfant ; la poésie et les images } \\
\text { Témoignage de deux poètes : Jean Tardieu et J. Valotton. }\end{array}$ \\
\hline MNAM & $\begin{array}{l}21 \text { avril } \\
\text { Turbulence cinématographique dans les années } \mathbf{1 9 2 0}^{(*)} \\
\text { Conférence de Patrick de Haas. }\end{array}$ \\
\hline $\begin{array}{l}\text { Revue } \\
\text { parlée }\end{array}$ & $\begin{array}{l}\text { Judaïsme et modernité viennoise }{ }^{(*)} \\
\text { Table ronde avec G. Zeissel, J.-M. Rey, A. Nouss. }\end{array}$ \\
\hline MNAM & $\begin{array}{l}24 \text { avril } \\
\text { La peinture de Klimt }{ }^{(*)} \\
\text { Conférence par le professeur Christian M. Nebehay. }\end{array}$ \\
\hline BPI & $\begin{array}{l}\text { Littérature anglaise venue d'ailleurs } \\
\text { avec J. Guiloineau, K. Ishiguro, J.-M. de Montremy, D. Olivier, M. Veron, N. } \\
\text { Zimmermann. Conversation-rencontre. Animation : J. Edwards. }\end{array}$ \\
\hline BPI & $\begin{array}{l}\text { Freud et Vienne } \\
\text { avec E. Laurent, C. Millot, N. Minor, F. Sauvagrat, B. This. Animation : René Lew. }\end{array}$ \\
\hline
\end{tabular}




\begin{tabular}{|c|c|}
\hline $\begin{array}{l}\text { Espace } \\
\text { séminaire }\end{array}$ & $\begin{array}{l}\text { Philosophie et histoire } \\
\text { avec P. Burke, R. Chartier, M. Ferro, D. Janicaud, G. Levi, F Savater, P. Veyne, O. } \\
\text { Mongin, G. Rauiet. }\end{array}$ \\
\hline BPI & $\begin{array}{l}25 \text { avril } \\
\text { Beckett dans le siècle } \\
\text { Colloque international à l'occasion de son } 80^{\mathrm{e}} \text { anniversaire. } \\
\text { Avec L. St J.Butler, G. Dumur, J.-J. Mayoux, A. Simon, A. Robbe-Grillet, J. Fletcher. }\end{array}$ \\
\hline \multirow[t]{2}{*}{$\begin{array}{l}\text { Espace } \\
\text { séminaire }\end{array}$} & Philosophie et histoire \\
\hline & $\begin{array}{l}26 \text { avril } \\
\text { Notes en marge } \\
\text { Émission publique de Claude Samuel (Radio-France) avec la collaboration de } \\
\text { Jacqueline Muller. } \\
\text { La France et ses musiques, avec J. Mongrédien, B. Didier, J.-P. Derrien, F.-B. Mâche. } \\
\text { Pianistes et légendes, avec A. Tubeuf, J. Spicket, B. Monsaingeon. }\end{array}$ \\
\hline BPI & $\begin{array}{l}\text { Beckett dans le siècle } \\
\text { Colloque international à l'occasion de son } 80^{\mathrm{e}} \text { anniversaire. } \\
\text { Avec E. Brater, B. Rosset, Y. Takahashi, J. Pilling, S. Brienza, D. McMillan, J. Calder, D. } \\
\text { Duckworth. }\end{array}$ \\
\hline BPI & $\begin{array}{l}27 \text { avril } \\
\text { Beckett dans le siècle } \\
\text { Colloque international à l'occasion de son anniversaire. } \\
\text { Avec S. E. Gontarski, W. Asmus, A. Rodriguez-Gago, K. Worth, E. Tophoven, R. } \\
\text { Federman, A. Tagliaferri, G. Scarpetta, J. Knowlson. }\end{array}$ \\
\hline BPI & $\begin{array}{l}28 \text { avril } \\
\text { Beckett dans le siècle } \\
\text { Colloque international à l'occasion de son } 80^{\mathrm{e}} \text { anniversaire. } \\
\text { Projection vidéo pour les participants au colloque. Avec R. Lamont, P. Chabert, T. } \\
\text { Bishop, M. Esslin, A. Libera, B. Poirot-Delpech, D. Sherzer. }\end{array}$ \\
\hline $\begin{array}{l}\text { Revue } \\
\text { parlée }\end{array}$ & $\begin{array}{l}2 \text { mai } \\
\text { Hermann Broch (1886-1951) } \\
\text { Ouverture du colloque par Rudolf Altmüller et Gérard Régnier. « Hermann Broch et } \\
\text { son temps » par A. Kern « La vision du réel chez Hermann Broch » par E. Kaufholz. }\end{array}$ \\
\hline $\begin{array}{l}\text { Revue } \\
\text { parlée }\end{array}$ & $\begin{array}{l}3 \text { mai } \\
\text { Hermann Broch, prosateur } \\
\text { «La pensée d'Hermann Broch à la lumière du poststructuralisme » par E. Kiss (en } \\
\text { allemand) } \\
\text { « La curieuse genèse d'un roman en nouvelles » par R. Thieberger. }\end{array}$ \\
\hline
\end{tabular}




\begin{tabular}{|c|c|}
\hline $\begin{array}{l}\text { Revue } \\
\text { parlée }\end{array}$ & $\begin{array}{l}4 \text { mai } \\
\text { Hermann Broch, poète et musicien } \\
\text { «Le pressentiment de la modernité » par G. Schlocker } \\
\text { «Broch, Barraqué, Heidegger » par P. Ozzard-Low (en anglais) } \\
\text { «Broch et Virgile » par M. Sarrabezolles } \\
\text { «Broch et la musique » par J.-P. Boyer } \\
\text { «Broch, traduit » par J.-M. Rabaté } \\
\text { «Une polyphonie de l'inachevé » avec la participation de J.-P. Bier, D. Jehl. } \\
\text { Conception et animation: Alfred Kern (en collaboration avec l'Institut culturel } \\
\text { autrichien). }\end{array}$ \\
\hline BPI & $\begin{array}{l}7 \text { mai } \\
\text { Histoire et télévision } \\
\text { Avec Ph. Alfonsi, F. Denel, Ph. Joutard, P. Sorlin. } \\
\text { Animation : C. Grunblatt. Conversation-rencontre. }\end{array}$ \\
\hline \multirow[t]{2}{*}{ BPI } & $\begin{array}{l}8 \text { mai } \\
\text { Vienne et l'obsession du féminin } \\
\text { Avec F. Gaillard, V. Théophilidès. } \\
\text { Animation : Ch. Buci-Glucksmann. Soirée viennoise. }\end{array}$ \\
\hline & $\begin{array}{l}14 \text { mai } \\
\text { Des techniques traditionnelles à la technologie } \\
\text { Avec A. Siza, P. Dubois, F. Jourda, G. Perraudin. } \\
\text { Animation : J.-C. Garcias. } \\
\text { Architecture et construction. }\end{array}$ \\
\hline BPI & $\begin{array}{l}\text { L'image cinéma } \\
\text { Cabinet de lecture autour de G. Deleuze, Y. Ishaghpour. } \\
\text { Animation : J. Leenhardt. }\end{array}$ \\
\hline BPI & $\begin{array}{l}15 \text { mai } \\
\text { La littérature berbère aujourd'hui } \\
\text { Avec A. Bounfour, S. Chaker, Y. Djafri, T. Djaout, M. Mammeri, K. Yacine. Animation : } \\
\text { N. Fares. }\end{array}$ \\
\hline $\begin{array}{l}\text { Revue } \\
\text { parlée }\end{array}$ & $\begin{array}{l}\text { Rencontre avec des écrivains sud-africains } \\
\text { avec M. Maponya, E. Mphahlele, S. Cépamia, M. Tiali. } \\
\text { Animation : P. Haski, en présence de M. Leiris. } \\
\text { (En collaboration avec l'Adec et le mouvement antiapartheid. Avec le concours de la } \\
\text { Direction du livre et de la lecture du ministère de la Culture). }\end{array}$ \\
\hline BPI/ & $\begin{array}{l}22 \text { mai } \\
\text { Le Matin de Paris } \\
1936 \\
\text { avec L. Bodin, G. Elgey, D. Mayer, B. Poirot-Delpech. } \\
\text { Débat animé par A. Spire. }\end{array}$ \\
\hline $\begin{array}{l}\text { Revue } \\
\text { parlée }\end{array}$ & $\begin{array}{l}23 \text { mai } \\
\text { Voix et chants berbères } \\
\text { Récital de Ch. Kheddam, Nouara. } \\
\text { En collaboration avec « La boîte aux documents ». }\end{array}$ \\
\hline
\end{tabular}




\begin{tabular}{|c|c|}
\hline & $\begin{array}{l}24 \text { mai } \\
\text { Notes en marge. Programme musical de France-Culture } \\
\text { Émission publique de Claude Samuel avec la collaboration de Jacqueline Muller. } \\
\text { Musique et fiction } \\
\text { Avec S. Berthier, M. Breitman, Y.-M. Ergal, G. Lambrichs, M.-C. Barrault. } \\
\text { L'Amérique de la modernité } \\
\text { avec Ph. Albéra, J.-Y. Bosseur, P. Méfano, H.-L. de la Grange. }\end{array}$ \\
\hline MNAM & $\begin{array}{l}28 \text { mai } \\
\text { La photographie surréaliste } \\
\text { Table ronde. }\end{array}$ \\
\hline $\begin{array}{l}\text { Revue } \\
\text { parlée }\end{array}$ & $\begin{array}{l}\text { Théâtre, folie et thérapie } \\
\text { avec J.-J. Gaussel. Présentation : Georges Baal. }\end{array}$ \\
\hline BPI & $\begin{array}{l}29 \text { mai } \\
\text { L'ère du faux } \\
\text { Autour de la revue Autrement. Animation : Pamela Tytell. } \\
\text { Conversation-rencontre. }\end{array}$ \\
\hline $\begin{array}{l}\text { Revue } \\
\text { parlée }\end{array}$ & $\begin{array}{l}\text { György Somlyo } \\
\text { Lecture de son dernier ouvrage Que cela en français et en hongrois. Avec A. Bosquet, } \\
\text { M. Deguy, N. Borgeant et M. Deleuze. }\end{array}$ \\
\hline $\begin{array}{l}\mathrm{BPI} / \text { Le Matin } \\
\text { de Paris }\end{array}$ & $\begin{array}{l}5 \text { juin } \\
\text { La littérature soviétique : trésors enfouis à profusion } \\
\text { avec L. Denis, E. Etkind, E. Limonov, A. Siniavsky, D. Fezemann. } \\
\text { Animation : Antoine Spire. }\end{array}$ \\
\hline $\begin{array}{l}\text { Histoire et } \\
\text { société }\end{array}$ & $\begin{array}{l}9 \text { juin } \\
\text { Les interrogations des catholiques devant le Front populaire } \\
\text { avec M. Rebérioux, R. Rémond. }\end{array}$ \\
\hline BPI & $\begin{array}{l}12 \text { juin } \\
\text { Mémoire et homosexualité } \\
\text { avec Ph. Boucher, D. Fernandez. }\end{array}$ \\
\hline BPI & Colloque « L'affiche et l'affichage » \\
\hline $\begin{array}{l}\text { Revue } \\
\text { parlée }\end{array}$ & $\begin{array}{l}\text { Exercices de la patience : } \\
\text { Cahiers de philosophie } \mathrm{n}^{\circ} 6 \text { et } \mathrm{n}^{\circ} 7 \text {, essais de neutre } \\
\text { Présentation : A. David, J. Rolland, F. Wybrands. }\end{array}$ \\
\hline $\begin{array}{l}\mathrm{BPI} / \text { Le Matin } \\
\text { de Paris }\end{array}$ & $\begin{array}{l}19 \text { juin } \\
\text { Histoire des perceptions de la Révolution française } \\
\text { avec J.-C. Bonnet. Animation : Antoine Spire. }\end{array}$ \\
\hline $\begin{array}{l}\text { Revue } \\
\text { parlée }\end{array}$ & $\begin{array}{l}23 \text { juin } \\
\text { Pour Henri Focillon } \\
\text { Présentation : André Chastel, avec J. Arrouye (à l'occasion de l'ouvrage paru dans la } \\
\text { collection « Cahiers pour un temps»). }\end{array}$ \\
\hline
\end{tabular}




\begin{tabular}{|c|c|}
\hline BPI & $\begin{array}{l}26 \text { juin } \\
\text { Le mécenat en France } \\
\text { avec J.-J. Rose, J. Seguela (et Coluche, J.-L. Godard et B. Kouchner, sous réserve). } \\
\text { Animation : Pierre-Bernard Soulier. }\end{array}$ \\
\hline $\mathrm{CCI}$ & Débat « Lieux de travail ». \\
\hline MNAM & $\begin{array}{l}17 \text { septembre } \\
\text { La sculpture moderne : les paradoxes de la mémoire } \\
\text { Conférence musée de Jacques Boullet. }\end{array}$ \\
\hline BPI & $\begin{array}{l}18 \text { septembre } \\
\text { La littérature suédoise } \\
\text { Débat animé par J.-M. de Montrémy. }\end{array}$ \\
\hline BPI & $\begin{array}{l}25 \text { septembre } \\
\text { L'Afrique noire est-elle condamnée au sous-développement? } \\
\text { avec J.-F. Bayard, R. Dumont, J. Giri, Y. Lacoste. } \\
\text { Débat animé par Antoine Spire. }\end{array}$ \\
\hline $\begin{array}{l}\text { Histoire et } \\
\text { société }\end{array}$ & $\begin{array}{l}26 \text { septembre } \\
\text { Nouveaux destins des intellectuels } \\
\text { Soirée débat organisée par la Cellule. }\end{array}$ \\
\hline MNAM & $\begin{array}{l}1^{\mathrm{er}} \text { octobre } \\
\text { Alberto Giacometti : toutes choses près loin } \\
\text { Conférence musée de David Sylvester. } \\
\text { Textes de Alberto Giacometti, lus par Jacques Dupin. }\end{array}$ \\
\hline BPI & $\begin{array}{l}\text { Le travail aujourd'hui : nouveaux rythmes, nouvelles perceptions } \\
\text { avec F. Rochat, E. Morin, G. Perrault, B. Kapp. }\end{array}$ \\
\hline BPI & $\begin{array}{l}2 \text { octobre } \\
\text { La science, ça m'intéresse ! } \\
\text { (à l'occasion de la parution de deux nouvelles collections pour enfants chez } \\
\text { Hachette). } \\
\text { Débat animé par Françoise Balibar. }\end{array}$ \\
\hline $\begin{array}{l}\text { Revue } \\
\text { parlée }\end{array}$ & $\begin{array}{l}\text { Noces noires de Werner Lambersy } \\
\text { Une création musicale de Annette Van de Gorne, avec B.-G. Lafabrie, M. Quaghebeur, } \\
\text { F. de Haes, H. Juin, Fano, Ch. Hublin, C. Herviant. }\end{array}$ \\
\hline MNAM & $\begin{array}{l}8 \text { octobre } \\
\text { Les commandes publiques et la sculpture in situ } \\
\text { Table ronde animée par M. Rowell. Avec J.-L. Daval, M. Deschamps, C. Franblin, D. } \\
\text { Semin, D. Zacharopoulos. }\end{array}$ \\
\hline BPI & $\begin{array}{l}\text { Revue parlée } 9 \text { octobre } \\
\text { Image et informatique } \\
\text { Débat animé par Didier Krajnc } \\
\text { (à l'occasion de l'exposition Images codées). }\end{array}$ \\
\hline
\end{tabular}




\begin{tabular}{|c|c|}
\hline $\mathrm{CCI}$ & $\begin{array}{l}15 \text { octobre } \\
\text { Nouveaux espaces culturels } \\
\text { Des architectes dialoguent avec des écrivains, musiciens, metteurs en scène, } \\
\text { muséologues... (Autour du projet «Restauration et réaménagement du Château de } \\
\text { Rivoli à Turin »). Rencontre Architecture et construction. }\end{array}$ \\
\hline BPI & $\begin{array}{l}16 \text { octobre } \\
\text { Christian Bourgois, } 25 \text { ans d'édition } \\
\text { Débat animé par Michel Nuridsany. }\end{array}$ \\
\hline BPI & $\begin{array}{l}22 \text { octobre } \\
\text { Forum des écrivains pour la jeunesse } \\
\text { 1. Rencontre enfants, auteurs, éditeurs } \\
\text { 2. Rencontres presse et écrivains pour la jeunesse }\end{array}$ \\
\hline $\mathrm{CCI}$ & $\begin{array}{l}\text { Nouveaux espaces culturels } \\
\text { Des architectes dialoguent avec des écrivains, musiciens, muséologues, metteurs en } \\
\text { scène... Rencontre Architecture et construction. } \\
\text { Autour des projets de la « Maison des arts et de la culture à Chambéry » et de la } \\
\text { «Maison du livre, de l'image et du son à Villeurbanne». }\end{array}$ \\
\hline BPI & $\begin{array}{l}23 \text { octobre } \\
\text { Forum des écrivains pour la jeunesse } \\
\text { Rencontre enfants, auteurs, éditeurs. }\end{array}$ \\
\hline BPI & $\begin{array}{l}\text { Image d'Amérique } \\
\text { Débat animé par Pamela Tytell. }\end{array}$ \\
\hline BPI & $\begin{array}{l}24 \text { octobre } \\
\text { Forum des écrivains pour la jeunesse } \\
\text { Rencontre enfants, auteurs, éditeurs. }\end{array}$ \\
\hline BPI & $\begin{array}{l}25 \text { octobre } \\
\text { Forum des écrivains pour la jeunesse } \\
\text { Rencontre enfants, auteurs, éditeurs. }\end{array}$ \\
\hline $\mathrm{CCI}$ & $\begin{array}{l}29 \text { octobre } \\
\text { Nouveaux espaces culturels } \\
\text { Rencontre Architecture et construction autour du projet "Théâtre musical " } \\
\text { commandé par le théâtre de la Scala de Milan. }\end{array}$ \\
\hline BPI & $\begin{array}{l}\text { Vers un seul art du spectacle : la télévision? } \\
\text { Débat dans le cadre de l'exposition-vente Pleins feux, les arts du spectacle. Animation A. } \\
\text { Spire. }\end{array}$ \\
\hline BPI & $\begin{array}{l}30 \text { octobre } \\
\text { Spectacle...Filmé } \\
\text { Débat dans le cadre de l'exposition-vente Pleins feux, les arts du spectacle. Animation F. } \\
\text { Lafon. }\end{array}$ \\
\hline
\end{tabular}




\begin{tabular}{|c|c|}
\hline BPI & $\begin{array}{l}31 \text { octobre } \\
\text { Écrire pour le spectacle } \\
\text { Débat dans le cadre de l'exposition-vente Pleins feux, les arts du spectacle. Animation A. } \\
\text { Spire. }\end{array}$ \\
\hline BPI & $\begin{array}{l}3 \text { novembre } \\
\text { Décors, décors... } \\
\text { Débat dans le cadre de l'exposition-vente Pleins feux, les arts du spectacle. Animation F. } \\
\text { Pascaud. }\end{array}$ \\
\hline BPI & $\begin{array}{l}5 \text { novembre } \\
\text { Le texte de théâtre } \\
\text { Débat dans le cadre de l'exposition-vente Pleins feux, tes arts du spectacle. }\end{array}$ \\
\hline BPI & $\begin{array}{l}\text { Cabinet de lecture. Autour de « Parages » et « Schibboleth » de J. Derrida et Ph. } \\
\text { Lacoue-Labarthe. } \\
\text { Présenté et animé par Ch. Buci-Glucksmann, avec F. Gaillard. }\end{array}$ \\
\hline BPI & $\begin{array}{l}6 \text { novembre } \\
\text { L'individu comme spectacle } \\
\text { Débat dans le cadre de l'exposition-vente Pleins feux, les arts du spectacle. Animation F. } \\
\text { Pascaud. }\end{array}$ \\
\hline $\begin{array}{l}\text { Revue } \\
\text { parlée }\end{array}$ & $\begin{array}{l}\text { Le courant néo-nationaliste dans le Japon contemporain } \\
\text { Rencontre avec Kato Shuichi. Conférence suivie d'un débat. }\end{array}$ \\
\hline $\begin{array}{l}\text { Histoire et } \\
\text { société }\end{array}$ & $\begin{array}{l}7 \text { novembre } \\
\text { La mutation des rapports patrons/salariés } \\
\text { avec A. Lyon-Caen, J. Moreau, J.-L. Moynot, F. Perrin, A. Wahl, H. Weber. }\end{array}$ \\
\hline CCI & $\begin{array}{l}8 \text { novembre } \\
\text { Négocier l'entité paysagère } \\
\text { avec B. Lassus, L. Kroll, V. Gregotti, Ch. Belfante. Conférence-débat patronnée par la } \\
\text { revue Urbanisme. }\end{array}$ \\
\hline BPI & $\begin{array}{l}\text { Écritures contemporaines et publiques } \\
\text { Débat dans le cadre de l'exposition-vente Pleins feux, les arts du spectacle. }\end{array}$ \\
\hline $\begin{array}{l}\text { Revue } \\
\text { parlée }\end{array}$ & $\begin{array}{l}12 \text { novembre } \\
\text { «Mois, Borges » } \\
\text { Hommage à Jorge Luis Borges. «Les images de la femme dans l'œuvre de Borges ». } \\
\text { Conférence de M.-A. Meizoso. }\end{array}$ \\
\hline BPI & $\begin{array}{l}13 \text { novembre } \\
\text { Les revues dans la vie intellectuelle } \\
\text { Débat animé par Antoine Spire. Autour des revues Autrement, le Débat, Esprit, Les } \\
\text { Temps modernes, Traverses. }\end{array}$ \\
\hline $\begin{array}{l}\text { Revue } \\
\text { parlée }\end{array}$ & $\begin{array}{l}\text { «Mois, Borges " } \\
\text { Hommage à Jorge Luis Borges. Les Journées et les nuits, le Passé qui ne menace pas. Films } \\
\text { de J.-M. Bersoza. Présentation : A. Camp. }\end{array}$ \\
\hline
\end{tabular}




\begin{tabular}{|c|c|}
\hline BPI & $\begin{array}{l}14 \text { novembre } \\
\text { Le livre d'enfants à la télévision }\end{array}$ \\
\hline $\begin{array}{l}\text { Revue } \\
\text { parlée }\end{array}$ & $\begin{array}{l}15 \text { novembre } \\
\text { Pansémiotique et relativité absolue } \\
\text { Conférence de B. Duval et R. Sünder suivie d'un débat avec Ben, G. Bonieux, J. } \\
\text { Ducorroy, B. Gerboud, R. Hains, J. de la Villeglé... }\end{array}$ \\
\hline MNAM & $\begin{array}{l}17 \text { novembre } \\
\text { Giacometti : retour à la figuration } \\
\text { Conférence musée du professeur Reinhold Hohl. }\end{array}$ \\
\hline $\begin{array}{l}\text { Revue } \\
\text { parlée }\end{array}$ & $\begin{array}{l}\text { La philosophie de Nishida : un projet de dépasser la modernité et la philosophie } \\
\text { occidentale } \\
\text { Rencontre avec Yujiro Nakamura. }\end{array}$ \\
\hline $\mathrm{CCI}$ & $\begin{array}{l}19 \text { novembre } \\
\text { Nouveaux espaces culturels } \\
\text { Rencontre Architecture et construction autour du projet « Espaces polyvalents... » }\end{array}$ \\
\hline BPI & $\begin{array}{l}20 \text { novembre } \\
\text { L'édition d'entreprise : une expérience italienne } \\
\text { Débat avec J. Bonnet, J. Gattegno, F. Robichon, J.-J. Rose. }\end{array}$ \\
\hline $\begin{array}{l}\text { Revue } \\
\text { parlée }\end{array}$ & $\begin{array}{l}22 \text { novembre } \\
\text { Arts semiotica : autour d'A.J. Greimas } \\
\text { 1. La sémiotique dans la cité. Avec D. Bertrand, Th. Bösiguer, M. Castellana, Y. } \\
\text { Darrault, J.-M. Floch, M. Hammad, C. Zilberberg. } \\
\text { 2. Le sens d'un parcours. Avec J.-F. Allilaire, J.-C. Coquet, J.-P. Desclées, M. Detienne, } \\
\text { G. Genette, B. Latour, L. Marin. }\end{array}$ \\
\hline MNAM & $\begin{array}{l}24 \text { novembre } \\
\text { Turbulence cinématographique dans les années } 1920 \\
\text { Conférence musée de Patrick de Hass. }\end{array}$ \\
\hline CCI & $\begin{array}{l}26 \text { novembre } \\
\text { Nouveaux espaces culturels } \\
\text { Rencontre Architecture et construction autour du projet «Réaménagement du } \\
\text { théâtre municipal à Belfort». }\end{array}$ \\
\hline BPI & $\begin{array}{l}27 \text { novembre } \\
\text { La littérature italienne aujourd'hui } \\
\text { Débat avec M. David, M. Fusco, J.-B. Para, D. Sallenave. } \\
\text { Animation: A. Spire. }\end{array}$ \\
\hline MNAM & $\begin{array}{l}\text { L'art contemporain dans la presse écrite } \\
\text { Table ronde musée. Modérateur: } P \text {. Bouteiller. }\end{array}$ \\
\hline
\end{tabular}




\begin{tabular}{|c|c|}
\hline $\begin{array}{l}\text { Revue } \\
\text { parlée }\end{array}$ & $\begin{array}{l}28 \text { novembre } \\
\text { Le siècle d'Ezra Pound } \\
\text { Présentation : Jean-Michel Rabaté. } \\
\text { 1. Ezra Pound, aujourd'hui. Avec M. de Rachewiltz, Ch. Brooke-Rose. } \\
\text { 2. Ezra Pound et la musique. Par C. Whitehouse. } \\
\text { 3. Ezra Pound et la Chine. Par J. Shapiro. } \\
\text { 4. Ezra Pound à Paris. Par P. Mikriammos. } \\
\text { 5. Ezra Pound et l'Italie. Par M. Bacigalupo. }\end{array}$ \\
\hline BPI & $\begin{array}{l}3 \text { décembre } \\
\text { Cabinet de lecture : esthétique du voir } \\
\text { avec Giorgio Amgamben, Christine Buci-Glucksmann, Marc Le Bot, René Major. }\end{array}$ \\
\hline BPI & $\begin{array}{l}4 \text { décembre } \\
\text { Colloque international pour le } 30^{\mathrm{e}} \text { anniversaire des Études tsiganes } \\
\text { Musiques tsiganes, création et tradition. } \\
\text { Animation : Michel David. }\end{array}$ \\
\hline BPI & $\begin{array}{l}5 \text { décembre } \\
\text { Colloque international pour le } 30^{\mathrm{e}} \text { anniversaire des Études tsiganes } \\
\text { La place des tsiganes dans la cité ; traditions/évolutions. La langue. }\end{array}$ \\
\hline BPI & $\begin{array}{l}6 \text { décembre } \\
\text { Colloque international pour le } \mathbf{3 0} \text { anniversaire des Études tsiganes } \\
\text { Intégration ou confrontation; les manifestations culturelles. }\end{array}$ \\
\hline MNAM & $\begin{array}{l}11 \text { décembre } \\
\text { Rencontre avec le groupe Gutaï } \\
\text { Table ronde musée. }\end{array}$ \\
\hline $\begin{array}{l}\text { Espace } \\
\text { séminaire }\end{array}$ & $\begin{array}{l}\text { Interrogation démocratique et lien social } \\
\text { avec Bronislaw Baczko, Laurent Cohen-Tanugi, Jacques Donzelot, Jean-Marie } \\
\text { Goulemot, Krzystof Pomian, Richard Rorty et Madame Claude Imbert. }\end{array}$ \\
\hline $\begin{array}{l}\text { Revue } \\
\text { parlée }\end{array}$ & $\begin{array}{l}13 \text { décembre } \\
\text { La critique moderne et post-moderne au Japon } \\
\text { Table ronde avec Asada Akira, Hasumi Shigehiko, Karatani Kôjin et Félix Guattari. }\end{array}$ \\
\hline $\begin{array}{l}\text { Revue } \\
\text { parlée }\end{array}$ & $\begin{array}{l}\text { Abjection : marginalité et tradition } \\
\text { Rencontre avec Nakagami Kenji, Jacques Derrida. }\end{array}$ \\
\hline $\begin{array}{l}\text { Revue } \\
\text { parlée }\end{array}$ & $\begin{array}{l}14 \text { décembre } \\
\text { La critique moderne et post-moderne au Japon } \\
\text { Table ronde avec Asada Akira, Hasumi Shigehiko, Karatani Kôjin. }\end{array}$ \\
\hline $\begin{array}{l}\text { Revue } \\
\text { parlée }\end{array}$ & $\begin{array}{l}15 \text { décembre } \\
\text { Le vent se lève } \\
\text { Par Imai Toshimitsu. Avec Jacques Dupin, Jean-Pierre Faye, Félix Guattari, A. } \\
\text { Jouffroy. }\end{array}$ \\
\hline
\end{tabular}




\begin{tabular}{|l|l|}
\hline CCI & $\begin{array}{l}17 \text { décembre } \\
\text { Nouveaux espaces culturels } \\
\text { Rencontre architecture et construction autour du projet : « La Neue Staats Galerie à } \\
\text { Stuttgart » avec James Stirling, Madeleine Reberioux. }\end{array}$ \\
\hline BPI & $\begin{array}{l}\text { Place et rôle de Lu Xun dans la littérature et la société chinoises } \\
\text { contemporaines } \\
\text { Débat à l'occasion du } 50^{e} \text { anniversaire de la mort de Lu Xun. }\end{array}$ \\
\hline $\begin{array}{l}\text { Revue } \\
\text { parlée }\end{array}$ & $\begin{array}{l}\text { Le Haiku contemporain } \\
\text { Conférence d'André Delteil. Lecture par Daniel Zerki. }\end{array}$ \\
\hline $\begin{array}{l}\text { Revue } \\
\text { parlée }\end{array}$ & $\begin{array}{l}\text { Le Renga : création collective au Japon et ailleurs } \\
\text { avec Jean-Pierre Faye, Alain Jouffroy, Jacques Roubaud. Lecture par Daniel Zerki. }\end{array}$ \\
\hline BPI & $\begin{array}{l}\text { Hommage à Jean-Marie Serreau } \\
\text { Débat animé par André Perinetti. Avec René Depestre, Kateb Yacine. }\end{array}$ \\
\hline
\end{tabular}

(*) Dans le cadre de l'exposition Vienne 1880-1938.

\section{Annexe 4. Revues qui ont organisé des débats}

$\begin{array}{ll}28 & \text { Aléa } \\ 29 & \text { Aires } \\ 30 & \text { L'Ane } \\ 31 & \text { Arguments } \\ 32 & \text { Autrement } \\ 33 & \text { Babylone } \\ 34 & \text { Banana split } \\ 35 & \text { Conséquences } \\ 36 & \text { Digraphe } \\ 37 & \text { Dirty } \\ 38 & \text { Doc(k)s } \\ 39 & \text { Documents } \\ 40 & \text { L'Ennemi } \\ 41 & \text { Esprit } \\ 42 & \text { Europe } \\ 43 & \text { Frank } \\ 44 & \text { Hérodote } \\ 45 & \text { Logomotives } \\ 46 & \text { Obsidiane } \\ 47 & \text { Ouilaphilosophie }\end{array}$


48 Phantomas

49 Pictura Edelweiss

50 Poémonde

51 Race \& Class

52 Textuerre

\section{NOTES}

1. IFA : Institut français d'architecture. 


\section{Remerciements}

1 Nous remercions les personnes suivantes qui ont bien voulu répondre à nos questions à la Bibliothèque publique d'information (Bpi) :

\begin{tabular}{|l|l|}
\hline P. Arbaizar & A. Meyer \\
\hline M. Benhamou & D. Reynier \\
\hline A.-M. Blanc & C. Stra \\
\hline T. Cremel & C. Timsit \\
\hline F. Figuière & J.-L. Villard \\
\hline M. Melot & \\
\hline
\end{tabular}

2 au Centre de création industrielle (CCI) :

\begin{tabular}{|l|l|}
\hline J. Chapelle & R. Guidot \\
\hline A. Guiheux & M.-L. Jousset \\
\hline C. Eveno & V. Hahn \\
\hline
\end{tabular}

au Musée national d'art moderne (MNAM) :

4 J.-H. Martin

5 C. Lawless

6 à la « Revue parlée » :

7 B. Gautier C. Valentin

8 M.-T. Angneroh

9 à l'Espace-séminaire : 
10 C. Descamps

11 à la cellule Histoire et société :

12 R. Rotmann

13 à la Cellule liaison-adhésion :

14 C. Fourteau

15 au service des archives :

16 H. de Langle 